\title{
Gravitational waves from pulsars in the context of magnetic ellipticity
}

\author{
José C. N. de Araujo ${ }^{\mathrm{a}}$, Jaziel G. Coelho ${ }^{\mathrm{b}}$, Cesar A. Costa ${ }^{\mathrm{c}}$ \\ Divisão de Astrofísica, Instituto Nacional de Pesquisas Espaciais, Avenida dos Astronautas 1758, São José dos Campos, SP 12227-010, Brazil
}

Received: 14 February 2017 / Accepted: 14 May 2017 / Published online: 26 May 2017

(C) The Author(s) 2017. This article is an open access publication

\begin{abstract}
In one of our previous articles we have considered the role of a time dependent magnetic ellipticity on the pulsars' braking indices and on the putative gravitational waves these objects can emit. Since only nine of more than 2000 known pulsars have accurately measured braking indices, it is of interest to extend this study to all known pulsars, in particular as regards gravitational wave generation. To do so, as shown in our previous article, we need to know some pulsars' observable quantities such as: periods and their time derivatives, and estimated distances to the Earth. Moreover, we also need to know the pulsars' masses and radii, for which we are adopting current fiducial values. Our results show that the gravitational wave amplitude is at best $h \sim 10^{-28}$. This leads to a pessimistic prospect for the detection of gravitational waves generated by these pulsars, even for Advanced LIGO and Advanced Virgo, and the planned Einstein Telescope, if the ellipticity has a magnetic origin.
\end{abstract}

\section{Introduction}

It is well known that, besides compact binaries, rapidly rotating neutron stars are promising sources of gravitational waves (GWs) which could be detected in a near future by advanced LIGO (aLIGO) and advanced VIRGO (AdV), and also by the planned Einstein Telescope (ET). These sources generate continuous GWs whether or not they are perfectly symmetric around their rotation axis, i.e., they show some equatorial ellipticity.

It is worth stressing that the equatorial ellipticity is an extremely relevant parameter since the GW amplitude is directly proportional to it. Therefore, if the ellipticity be extremely small, i.e., $\varepsilon \ll 10^{-5}$, the GW amplitude will also

\footnotetext{
a e-mail: jcarlos.dearaujo@inpe.br

be-mail: jaziel.coelho@inpe.br

ce-mail: cesar.costa@inpe.br
}

be extremely small, implying that the detection of such continuous GWs generated by pulsars may be unattainable (see, $[1,2])$ with current technology. For comparison, some authors argue that an acceptable upper limit for the ellipticity would be around $\varepsilon \sim 10^{-6}$ (see, e.g., [3]). An important mechanism for producing asymmetries is the development of nonaxisymmetric instabilities in rapidly rotating neutron stars driven by the gravitational emission reaction or by nuclear matter viscosity (see, e.g., [4], and the references therein).

We explore, in the present paper, some consequences of an ellipticity generated by the magnetic dipole of the pulsars themselves. It is well known that, for strong magnetic fields $\left(\sim 10^{12}\right.$ to $\left.10^{15} \mathrm{G}\right)$, the equilibrium configuration of a neutron star can be distorted due to the magnetic pressure. Therefore, both rotation and magnetic field combined can produce a flattened equilibrium star. However, star rotation and strong magnetic field may not be sufficient for GW emission, other effects must be associated with it, such as the pulsar's precession (see, e.g., [5]).

The main goal of the present paper is to extend our previous study where the role of ellipticity of magnetic origin $\left(\varepsilon_{\mathrm{B}}\right)$ was considered [6]. Since in that paper we were also interested in braking indices, which are until now accurately measured for only nine pulsars, we had restricted that study exclusively for those very pulsars.

However, $\varepsilon_{\mathrm{B}}$ does not depend on the pulsar braking index; maybe it is the other way around. In fact, $\varepsilon_{\mathrm{B}}$ is mostly associated to both the pulsar period $(P)$ and its time derivative $(\dot{P})$, for a given value of mass, radius and moment of inertia. Therefore, it is straightforward to extend the $\varepsilon_{\mathrm{B}}$ calculation for all pulsars with known $P$ and $\dot{P}$. Consequently, with $\varepsilon_{\mathrm{B}}$ in hands, we can calculate the GW amplitudes for all pulsars with known $P, \dot{P}$, and their distances to the Earth. In this regard it is worth mentioning that the distance to these sources has been established, although there are observational uncertainties in its determination, which are not taken into account in the present paper. A putative uncertainty in 
one order of magnitude in distance would mean this very uncertainty in the GW amplitude.

To do so this paper is organized as follows: Sect. 2 is devoted to a brief procedure description which is conducted by a basic set of equations. In Sect. 3 we present the calculations and discuss the results obtained. Finally, in Sect. 4 we summarize the main conclusions and make remarks about them.

\section{Basic equations}

In Ref. [6] we consider in detail how to relate $\varepsilon_{\mathrm{B}}$ to $P$ and $\dot{P}$. In addition, the basic equations used for calculating the amplitude of the putative GWs generated by pulsars are also presented. All those equations are used to calculate the relevant quantities of this present paper. Therefore, here we are only providing the main steps for deriving these relevant equations.

Recall that the equatorial ellipticity is given by (see e.g., [7])

$\varepsilon=\frac{I_{x x}-I_{y y}}{I_{z z}}$,

where $I_{x x}, I_{y y}, I_{z z}$ are the moment of inertia with respect to the rotation axis (hereafter simply denoted $I$ ), $z$, and along the directions perpendicular to it.

Regarding the ellipticity of magnetic origin, it was shown by different authors [8-10] to be given by

$\varepsilon_{\mathrm{B}}=\kappa \frac{B_{0}^{2} R^{4}}{G M^{2}} \sin ^{2} \phi$,

where $B_{0}$ is the dipole magnetic field, $R$ and $M$ are the radius and the mass of the star, respectively, $\phi$ is the angle between the rotation and magnetic dipole axes, whereas $\kappa$ is the distortion parameter, which depends on both the star equation of state $(\mathrm{EoS})$ and the magnetic field configuration (see e.g., [10]).

Regarding the GW amplitude, one finds in the literature the following equation:

$h^{\mathrm{SD}}=\left(\frac{5}{2} \frac{G}{c^{3}} \frac{I}{r^{2}} \frac{\left|\dot{f}_{\mathrm{rot}}\right|}{f_{\text {rot }}}\right)^{1 / 2}$

(see, e.g., [11]) where one considers the whole contribution to $\dot{f}_{\text {rot }}$ to be due to $\mathrm{GW}$ emission, i.e., we have the spin-down limit. This equation must be modified to take into account the magnetic braking (see $[1,2]$ ). This can be done by writing

$\dot{\bar{f}}_{\text {rot }}=\eta \dot{f}_{\text {rot }}$, where $\dot{\bar{f}}_{\text {rot }}$ can be interpreted as the part of $\dot{f}_{\text {rot }}$ related to the GW emission brake. Consequently, the GW amplitude is given by

$h^{2}=\frac{5}{2} \frac{G}{c^{3}} \frac{I}{r^{2}} \frac{\left|\dot{\bar{f}}_{\text {rot }}\right|}{f_{\text {rot }}}=\frac{5}{2} \frac{G}{c^{3}} \frac{I}{r^{2}} \frac{\left|\dot{f}_{\text {rot }}\right|}{f_{\text {rot }}} \eta$.

On the other hand, the GWs amplitude can also be written as follows:

$h=\frac{16 \pi^{2} G}{c^{4}} \frac{I \varepsilon f_{\mathrm{rot}}^{2}}{r}$

(see, e.g., [7]). By combining the two equations above one can obtain $\varepsilon_{\mathrm{B}}$ in terms of $P, \dot{P}$ (observable quantities), $\eta$ and $I$, namely

$\varepsilon=\sqrt{\frac{5}{512 \pi^{4}} \frac{c^{5}}{G} \frac{\dot{P} P^{3}}{I} \eta}$.

Still concerning $\eta$, as discussed in detail by [6], it can be also be interpreted as the fraction of the rotation power $\left(\dot{E}_{\text {rot }}\right)$ emitted in the form of GWs $\left(\dot{E}_{\mathrm{GW}}\right)$, or yet, the efficiency for GW generation. Obviously, part of the rotation power is emitted in the form of electromagnetic radiation through magnetic dipole emission $\left(\dot{E}_{\mathrm{d}}\right)$.

Also, in [6] an useful equation is shown relating $\eta$ to the pulsar dipole magnetic field, which is derived by recalling that the magnetic brake is related to $P$ and $\dot{P}$, i.e.

$\bar{B}_{0} \sin ^{2} \phi=\frac{3 I c^{3}}{4 \pi^{2} R^{6}} P \dot{P}$

where $\bar{B}_{0}$ would be the magnetic field if the breaking is purely magnetic. Since pulsars might also emit GWs, $B_{0}<\bar{B}_{0}$ is a reasonable assumption. Thus, the equation relating $\eta$ to the pulsar dipole magnetic field reads (see [6] for details)

$\eta=1-\left(\frac{B_{0}}{\bar{B}_{0}}\right)^{2}$.

By substituting this last expression into Eq. (2), one obtains

$\varepsilon_{\mathrm{B}}=\frac{3 I c^{3}}{4 \pi^{2} G M^{2} R^{2}} P \dot{P}(1-\eta) \kappa$.

In addition, by combining this equation with Eq. (7), one has

$\eta=\frac{288}{5} \frac{I^{3} c}{G M^{4} R^{4}} \frac{\dot{P}}{P}(1-\eta)^{2} \kappa^{2}$. 


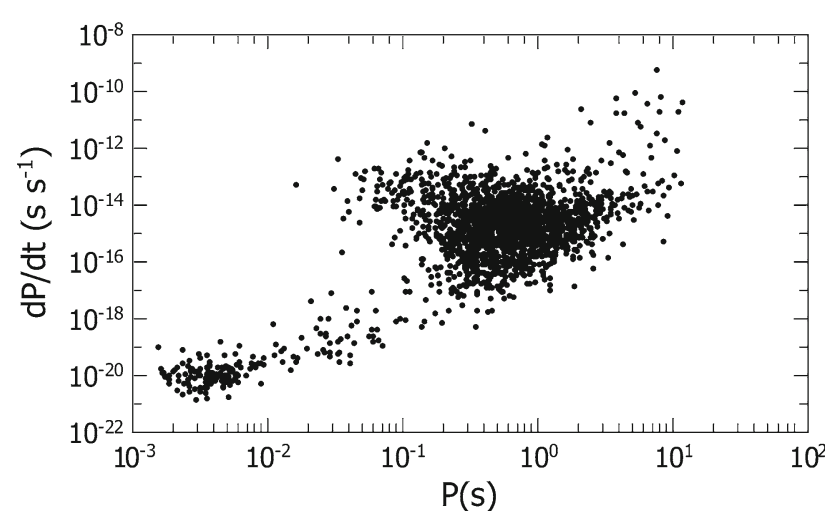

Fig. 1 The $P-\dot{P}$ diagram for radio pulsars obtained from the ATNF Pulsar Catalog (http://www.atnf.csiro.au/people/pulsar/psrcat/)

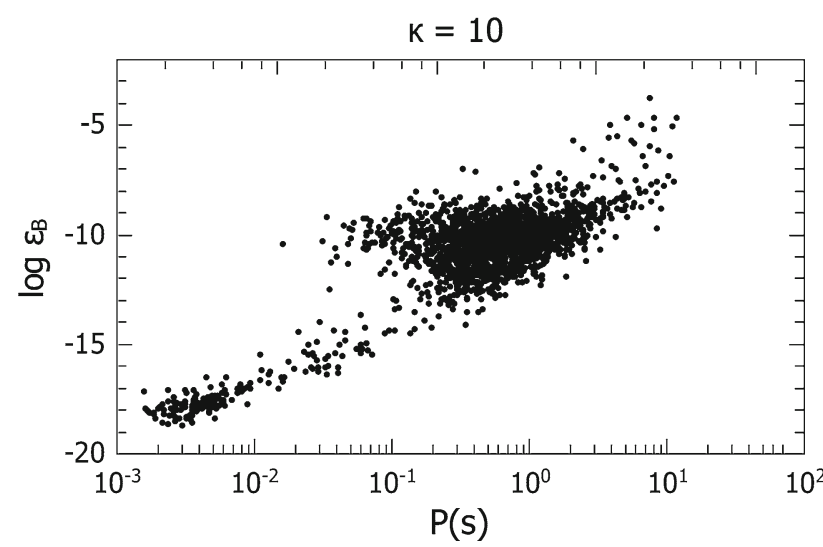

Fig. 2 Correlation between the magnetic ellipticity and the period for all pulsars with known $P, \dot{P}$

Since $\varepsilon$ and $\eta \ll 1$, one can readily obtain the following useful equations:

$\varepsilon_{\mathrm{B}} \simeq \frac{3 I c^{3}}{4 \pi^{2} G M^{2} R^{2}} P \dot{P} \kappa$

and

$\eta \simeq \frac{288}{5} \frac{I^{3} c}{G M^{4} R^{4}} \frac{\dot{P}}{P} \kappa^{2}$.

Now, we are ready to calculate $\varepsilon_{\mathrm{B}}, \eta$ and the GW amplitudes for all pulsars with known $P, \dot{P}$, and estimated distances to the Earth, for given values of $M, R, I$ and $\kappa$. The next section is devoted to such an issue as well as the corresponding discussion of the results.

\section{Calculations and discussions}

The table with the necessary parameters used for the calculation of $\varepsilon_{\mathrm{B}}, \eta$ and $h$ as well as the adopted criteria for the selection of the pulsars can be found in the appendix. Fig-

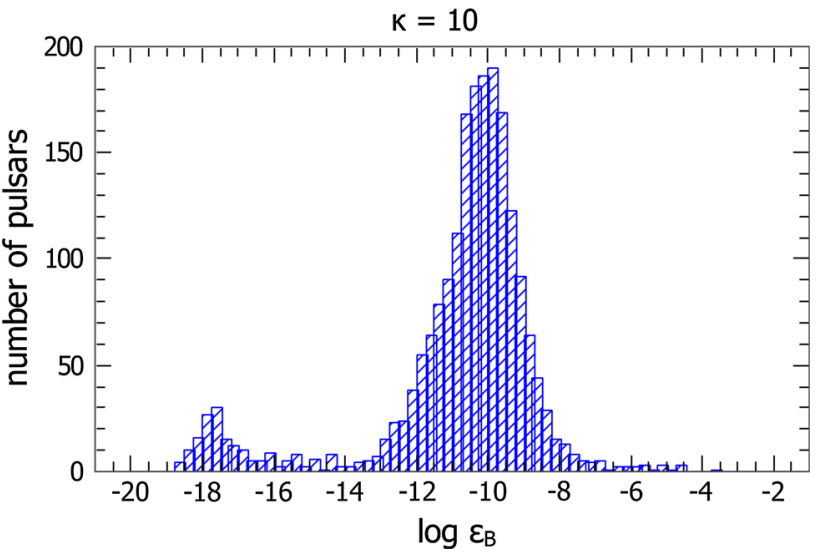

Fig. 3 Ellipticity histogram for the 1964 pulsars of Table 1 for $k=10$

ure 1 is made with columns 2 and 3 of this very table, in which the pulsars' periods and their corresponding time derivatives (spin-down rate) are shown. Although being a well-known diagram, it will be very useful for the discussions of the subsequent results. It is easily distinguishable two pulsar populations: the millisecond pulsars in the lower left (with periods $P<10^{-2} \mathrm{~s}$ ), composed of very old pulsars, and another class of isolated pulsars with periods $10^{-1}<P<10^{1}$ s, composed of younger pulsars which have already depleted a respectful fraction of their rotation power.

On the last three columns of Table 1, we present the results of our calculations, namely, $\varepsilon_{\mathrm{B}}, \eta$ and $h$. In order to perform these calculations, we also need to provide values for $M$, $R, I$ and $\kappa$. For the first three parameters, we are adopting fiducial values, namely, $M=1.4 M_{\odot}, R=10 \mathrm{~km}$, and $I=$ $10^{38} \mathrm{~kg} \mathrm{~m}^{2}$. Regarding the distortion parameter $\kappa$, as already mentioned, it depends on the EoS and on the magnetic field configuration. In particular, we choose $\kappa=10$, but values as high as $\kappa=1000$ could be considered, although they are probably unrealistic (see, e.g., [10] for a brief discussion).

From Eq. (12), we find that $\varepsilon_{\mathrm{B}}$ is extremely small $\left(\sim 10^{-19}\right.$ to $10^{-15}$ ) for the millisecond pulsars of Table 1, even when the extremely optimistic case in which $\kappa \sim 1000$ is considered. Moreover, the ellipticity distribution assumes values of the order of $\sim 10^{-10}$ for the slowest pulsars. In Fig. 2 we present $\log \varepsilon_{\mathrm{B}}$ versus $P$ for $\kappa=10$ for all pulsars of Table 1 . The ellipticity for different values of $\kappa$, since $\varepsilon_{\mathrm{B}} \propto \kappa$, can be readily obtained.

An interesting histogram can also be made from Table 1, namely, the number of pulsars for a $\log \varepsilon_{\mathrm{B}}$ bin (see Fig. 3). The high number of pulsars concentrated around $\sim 10^{-10}$ $\left(10^{-8}\right)$ for $k=10(1000)$ is worth noticing.

A similar analysis can be made for $\eta$ by means of Eq. (13). In Fig. 4 we present $\log \eta$ versus $P$ for $\kappa=10$ for all pulsars of Table 1. Notice that $\eta$ is also extremely small, even if one consider $\kappa=1000$. Also, an histogram for the number of pulsars for $\log \eta^{1 / 2}$ can be seen in Fig. 5. This quantity $\left(\eta^{1 / 2}\right)$ 


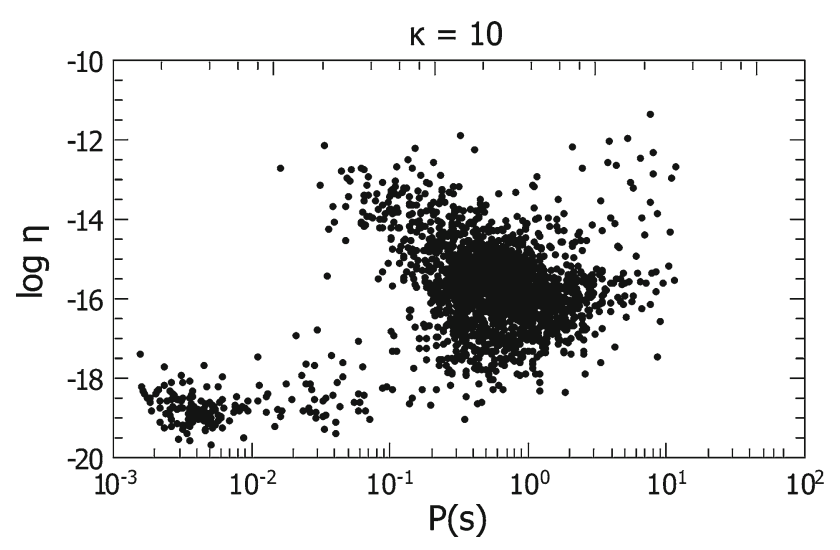

Fig. 4 Correlation between the efficiency and the period for all pulsars with known $P, \dot{P}$

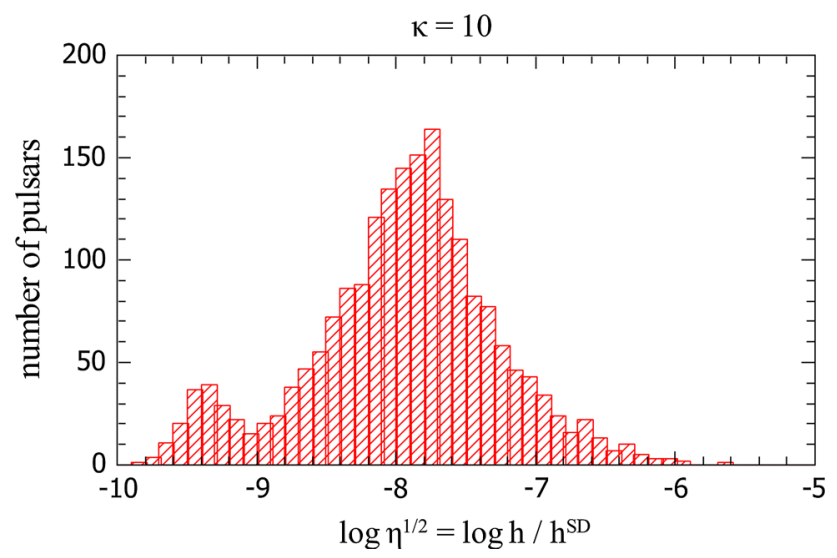

Fig. 5 Histogram of the efficiencies (or the spin-down ratio) for the 1964 pulsars of Table 1 for $k=10$

is equivalent to the ratio $h / h^{\mathrm{SD}}$, i.e., the spin-down ratio. One may notice a peak in the $\eta$ histogram at $10^{-16}$ to $10^{-15}$ for the pulsars of Table 1 . As for $\varepsilon_{\mathrm{B}}, \eta$ for different values of $\kappa$ can easily be obtained, since $\eta \propto \kappa^{2}$. Thus, even for $\kappa=1000$ the peak in the histogram would be around $10^{-12}-10^{-11}$. Before proceeding, it is worth stressing that the two pulsar populations mentioned above also clearly appear in Figs. 2, 3,4 and 5 .

These extremely small values of $\varepsilon_{\mathrm{B}}$ and $\eta$ have very important consequences as regards the detectability of GWs generated by the pulsars, if the ellipticity is mainly due to the magnetic dipole of the pulsars themselves. Our calculations show that the GW amplitudes for most of these pulsars are at best seven orders of magnitude smaller than those obtained by assuming the spin-down limit (SD); see Fig. 6. Notice that, even considering an extremely optimistic case, the value of the ellipticity is at best $\varepsilon_{\mathrm{B}} \sim 10^{-5}$ (for PSR J1846-0258) and the corresponding efficiency $\eta \sim 10^{-8}$. Thus, the GW amplitude even in this case would be four orders of magnitude lower than the amplitude obtained by assuming the spin-down limit $(\eta=1)$.

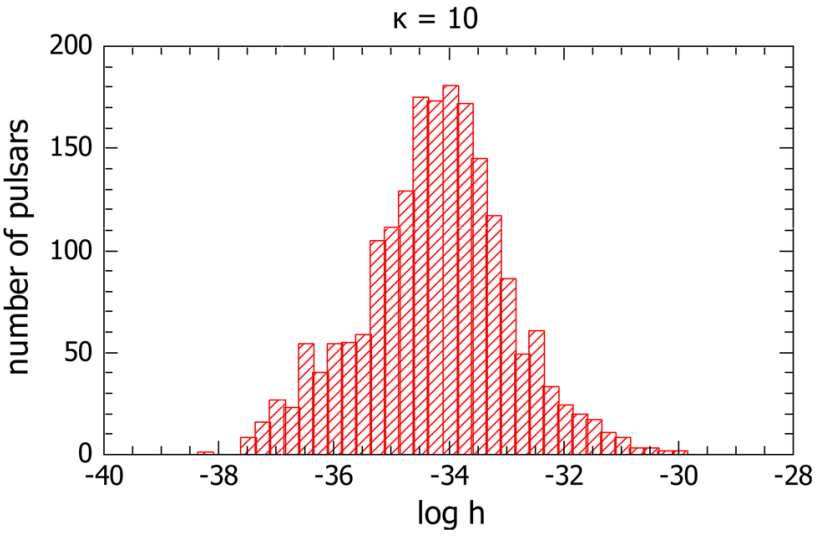

Fig. 6 Histogram of the amplitude of GWs for all pulsars with known $P, \dot{P}$

Still regarding Fig. 5, a recent result from the searches for GWs from 200 pulsars by using the aLIGO first observation run data (see Fig. 4 of [12]) presents a similar distribution for $h / h^{\mathrm{SD}}$, but displaced by some orders of magnitude. The high ellipticity values they found should be considered as upper limits since no continuous $\mathrm{GW}$ has been detected. In our present work, as already mentioned, we have considered that the ellipticity is caused exclusively by magnetic dipole and showed that such a mechanism alone could not generate a much strong star deformation. In our view, another mechanism or other mechanisms should be associated to the magnetic field to make possible those putative high ellipticities, e.g., high precession and/or non-conventional EoS.

For a matter of comparison, some authors argue that a fiducial upper limit for the ellipticity would be around $\varepsilon \sim 10^{-6}$, considering asymmetries supported by anisotropic stress built up during the crystallization period of the crust (see, e.g., [3], and references therein).

Finally, since the predicted GW amplitudes are extremely small for all pulsars of Table 1, and thousands of years of observing time would be needed, even advanced detectors such as aLIGO and AdV, and the planned ET would not be able to detect these pulsars, if the ellipticity is of magnetic dipole origin.

\section{Conclusions and final remarks}

In this paper, we extend our previous studies [6] in which we considered the role of magnetic ellipticity on the braking index and on the pulsar distortion. It is well known that, for strong magnetic fields $\left(\sim 10^{12}\right.$ to $\left.10^{15} \mathrm{G}\right)$, the equilibrium configuration of a neutron star can be distorted by magnetic tension. Such magnetic fields could not generate enough deformation to lead to high values of ellipticity. The presence of a magnetic field exceeding the magnetar strength 
$\left(\sim 10^{16} \mathrm{G}\right)$ could account for an ellipticity of $\sim 10^{-4}$. Recall that much higher values of magnetic fields could violate the Virial Theorem and disrupt the star equilibrium (see, e.g., [13]).

Here we consider the role played by the magnetic dipole field on the deformation of the known pulsars and its consequences as regards the generation of GWs. In particular, we obtained the useful Eqs. (12) and (13), with which one can calculate $\varepsilon_{\mathrm{B}}$ and $\eta$ in terms of $I, M, R, \kappa$ and the observable quantities $P$ and $\dot{P}$, and distances to the Earth. Moreover, the amplitudes of GWs can be readily calculated.

Regarding the GWs generated by the pulsars, our calculations show that the amplitudes are extremely small, as a result the prospect for their detection, even for aLIGO, AdV and the planned ET, would be pessimistic. This conclusion is obviously dependent on the mechanism that generates the ellipticity. If there is some other mechanism that could generate substantially larger ellipticities, the prospects for the detection of GWs emitted by the pulsars of Table 1 could be much less pessimistic.
Acknowledgements J.C.N.A thanks FAPESP (2013/26258-4) and CNPq (308983/2013-0 and 307217/2016-7) for partial support. J.G.C. acknowledges the support of FAPESP (2013/15088-0 and 2013/262584). C.A.C. acknowledges PNPD-CAPES for financial support.

Open Access This article is distributed under the terms of the Creative Commons Attribution 4.0 International License (http://creativecomm ons.org/licenses/by/4.0/), which permits unrestricted use, distribution, and reproduction in any medium, provided you give appropriate credit to the original author(s) and the source, provide a link to the Creative Commons license, and indicate if changes were made.

Funded by SCOAP ${ }^{3}$.

\section{Appendix}

Table 1 presents the periods $(P)$, their first derivatives $(\dot{P})$, and the estimated distances $(d)$ from Earth of 1964 pulsars drawn from the ATNF Pulsar Catalog (http://www.atnf.csiro. $\mathrm{au} /$ people/pulsar/psrcat/). Another selection criterion is that the pulsars must have only an unique measured value for $\dot{P}$. Also presented are values of $\eta, \varepsilon$ and $h$ calculated from the appropriate equations, which can be found in Sect. 3 .

Table $1 P, \dot{P}$ and $d$ for pulsars drawn from the ATNF catalog, with an unique measured value for $\dot{P}$, and the corresponding $\varepsilon, \eta$ and $h$ for $\kappa=10$

\begin{tabular}{|c|c|c|c|c|c|c|}
\hline Pulsar & $P(\mathrm{~s})$ & $\log \dot{P}$ & $d(\mathrm{kpc})$ & $\log \varepsilon$ & $\log \eta$ & $\log h$ \\
\hline $\mathrm{J} 0006+1834$ & 0.693748 & -14.6778 & 0.7 & -10.1931 & -15.7481 & -33.0943 \\
\hline $\mathrm{J} 0007+7303$ & 0.315873 & -12.4437 & 1.4 & -8.30073 & -13.1724 & -30.8195 \\
\hline J0014+4746 & 1.2407 & -15.2487 & 1.82 & -10.5116 & -16.5715 & -34.3327 \\
\hline $\mathrm{J} 0026+6320$ & 0.318358 & -15.8239 & 13.6 & -11.6775 & -16.556 & -35.1906 \\
\hline $\mathrm{J} 0030+0451$ & 0.004865 & -19.9914 & 0.3 & -17.6609 & -18.9076 & -35.8858 \\
\hline J0034-0534 & 0.001877 & -20.3036 & 0.98 & -18.3867 & -18.8063 & -36.2985 \\
\hline J0034-0721 & 0.942951 & -15.3893 & 1.03 & -10.7714 & -16.593 & -34.1069 \\
\hline $\mathrm{J} 0040+5716$ & 1.11823 & -14.5406 & 4.48 & -9.84862 & -15.8183 & -33.9706 \\
\hline J0045-7042 & 0.632336 & -14.6038 & 59.7 & -10.1594 & -15.6339 & -34.9109 \\
\hline J0045-7319 & 0.926276 & -14.3507 & 59.7 & -9.74047 & -15.5466 & -34.8236 \\
\hline $\mathrm{J} 0048+3412$ & 1.21709 & -14.6289 & 3.68 & -9.90016 & -15.9434 & -34.0103 \\
\hline $\mathrm{J} 0051+0423$ & 0.354732 & -17.1549 & 0.94 & -12.9615 & -17.934 & -35.4081 \\
\hline $\mathrm{J} 0055+5117$ & 2.11517 & -14.0205 & 2.4 & -9.05165 & -15.5749 & -33.4562 \\
\hline J0056+4756 & 0.472037 & -14.4776 & 1 & -10.1601 & -15.3807 & -32.8817 \\
\hline J0057-7201 & 0.738062 & -16 & 2.49 & -11.4885 & -17.0972 & -34.9945 \\
\hline J0100-7211 & 8.02039 & -10.7258 & 59.7 & -5.17819 & -12.8592 & -32.1362 \\
\hline J0101-6422 & 0.002573 & -20.2874 & 0.73 & -18.2335 & -18.9269 & -36.2913 \\
\hline $\mathrm{J} 0102+6537$ & 1.67916 & -14.2255 & 2.45 & -9.35694 & -15.6797 & -33.5699 \\
\hline $\mathrm{J} 0106+4855$ & 0.083157 & -15.3686 & 7.33 & -11.8052 & -15.5176 & -33.8838 \\
\hline J0108-1431 & 0.807565 & -16.1135 & 0.21 & -11.5629 & -17.2498 & -34.0731 \\
\hline $\mathrm{J} 0108+6608$ & 1.28366 & -13.8827 & 1.65 & -9.13083 & -15.2203 & -32.9389 \\
\hline $\mathrm{J} 0108+6905$ & 1.07112 & -16.3179 & 2.59 & -11.6446 & -17.5768 & -35.4912 \\
\hline J0111-7131 & 0.688542 & -14.1494 & 59.7 & -9.66797 & -15.2164 & -34.4935 \\
\hline J0113-7220 & 0.325883 & -14.3134 & 59.7 & -10.1568 & -15.0556 & -34.3326 \\
\hline $\mathrm{J} 0117+5914$ & 0.101439 & -14.2328 & 2.14 & -10.5832 & -14.4682 & -32.2997 \\
\hline
\end{tabular}


Table 1 continued

\begin{tabular}{|c|c|c|c|c|c|c|}
\hline Pulsar & $P(\mathrm{~s})$ & $\log \dot{P}$ & $d(\mathrm{kpc})$ & $\log \varepsilon$ & $\log \eta$ & $\log h$ \\
\hline $\mathrm{J} 0131-7310$ & 0.348124 & -14.7545 & 59.7 & -10.5693 & -15.5254 & -34.8024 \\
\hline J0133-6957 & 0.463474 & -15.9208 & 2.42 & -11.6113 & -16.816 & -34.7009 \\
\hline J0134-2937 & 0.136962 & -16.1057 & 1.78 & -12.3256 & -16.4714 & -34.2229 \\
\hline $\mathrm{J} 0137+1654$ & 0.414763 & -16.9136 & 2.51 & -12.6524 & -17.7606 & -35.6613 \\
\hline J0139+5621 & 1.77534 & -13.1018 & 5.54 & -8.20909 & -14.5803 & -32.8248 \\
\hline $\mathrm{J} 0139+5814$ & 0.272451 & -13.9706 & 2.6 & -9.89188 & -14.6351 & -32.5511 \\
\hline $\mathrm{J} 0141+6009$ & 1.22295 & -15.4078 & 2.3 & -10.677 & -16.7244 & -34.5872 \\
\hline $\mathrm{J} 0146+6145$ & 8.68899 & -11.7011 & 3.6 & -6.11872 & -13.8693 & -31.9266 \\
\hline $\mathrm{J} 0147+5922$ & 0.196321 & -15.5901 & 1.91 & -11.6536 & -16.1122 & -33.8943 \\
\hline J0151-0635 & 1.46467 & -15.3536 & 1.93 & -10.5444 & -16.7485 & -34.5351 \\
\hline $\mathrm{J} 0152+0948$ & 2.74665 & -14.7696 & 2.3 & -9.6873 & -16.4375 & -34.3003 \\
\hline J0152-1637 & 0.832742 & -14.8861 & 0.69 & -10.3221 & -16.0357 & -33.3756 \\
\hline J0156+3949 & 1.81156 & -15.8182 & 4.85 & -10.9167 & -17.3054 & -35.4922 \\
\hline $\mathrm{J} 0157+6212$ & 2.35175 & -12.7235 & 1.61 & -7.70869 & -14.3241 & -32.032 \\
\hline $\mathrm{J} 0201+7005$ & 1.34918 & -14.2588 & 1.15 & -9.48533 & -15.6181 & -33.1798 \\
\hline J0205+6449 & 0.065716 & -12.7122 & 3.2 & -9.25107 & -12.759 & -30.7652 \\
\hline J0206-4028 & 0.63055 & -14.9208 & 0.88 & -10.4776 & -15.9497 & -33.3952 \\
\hline J0211-8159 & 1.07733 & -15.5376 & 1.83 & -10.8618 & -16.7991 & -34.5626 \\
\hline $\mathrm{J} 0212+5222$ & 0.376386 & -14.1805 & 1.91 & -9.96137 & -14.9852 & -32.7673 \\
\hline $\mathrm{J} 0214+5222$ & 0.024575 & -18.5243 & 1.21 & -15.4904 & -18.144 & -35.7278 \\
\hline $\mathrm{J} 0215+6218$ & 0.54888 & -15.1791 & 3.19 & -10.7962 & -16.1478 & -34.1526 \\
\hline $\mathrm{J} 0218+4232$ & 0.002323 & -19.1113 & 3.15 & -17.1018 & -17.7065 & -35.7058 \\
\hline $\mathrm{J} 0231+7026$ & 1.46682 & -14.5072 & 2.25 & -9.69741 & -15.9028 & -33.756 \\
\hline J0248+6021 & 0.217094 & -13.2588 & 2 & -9.27875 & -13.8246 & -31.6267 \\
\hline J0255-5304 & 0.447708 & -16.5143 & 1.15 & -12.2198 & -17.3944 & -34.9562 \\
\hline J0304+1932 & 1.38758 & -14.8861 & 0.95 & -10.1003 & -16.2575 & -33.7362 \\
\hline $\mathrm{J} 0323+3944$ & 3.03207 & -15.1965 & 2.61 & -10.0714 & -16.9074 & -34.8251 \\
\hline J0324+5239 & 0.33662 & -15.4191 & 6.28 & -11.2485 & -16.1754 & -34.4744 \\
\hline $\mathrm{J} 0329+1654$ & 0.89332 & -15.6676 & 3.35 & -11.0731 & -16.8477 & -34.8738 \\
\hline $\mathrm{J} 0332+5434$ & 0.71452 & -14.6882 & 1 & -10.1908 & -15.7714 & -33.2725 \\
\hline $\mathrm{J} 0335+4555$ & 0.269201 & -17.1337 & 2.08 & -13.0602 & -17.7929 & -35.6121 \\
\hline $\mathrm{J} 0337+1715$ & 0.002733 & -19.752 & 1.3 & -17.6719 & -18.4178 & -36.0328 \\
\hline J0343-3000 & 2.59703 & -16.2441 & 2.22 & -11.1862 & -17.8878 & -35.7352 \\
\hline $\mathrm{J} 0343+5312$ & 1.93448 & -13.8729 & 2.48 & -8.94288 & -15.3886 & -33.2841 \\
\hline $\mathrm{J} 0348+0432$ & 0.039123 & -18.618 & 2.1 & -15.3821 & -18.4396 & -36.2628 \\
\hline $\mathrm{J} 0357+5236$ & 0.19703 & -15.3215 & 4.73 & -11.3835 & -15.8452 & -34.0211 \\
\hline $\mathrm{J} 0358+5413$ & 0.156382 & -14.3565 & 1 & -10.5189 & -14.7799 & -32.2809 \\
\hline J0401-7608 & 0.545253 & -14.8125 & 1.5 & -10.4324 & -15.7782 & -33.4554 \\
\hline $\mathrm{J} 0406+6138$ & 0.594576 & -14.2541 & 3.05 & -9.83648 & -15.2575 & -33.2429 \\
\hline $\mathrm{J} 0407+1607$ & 0.025702 & -19.1024 & 4.07 & -16.049 & -18.7415 & -36.8521 \\
\hline $\mathrm{J} 0415+6954$ & 0.390715 & -16.1158 & 1.57 & -11.8805 & -16.9368 & -34.6337 \\
\hline J0418-4154 & 0.757119 & -15.0114 & 2.46 & -10.4888 & -16.1198 & -34.0117 \\
\hline $\mathrm{J} 0421-0345$ & 2.16131 & -14.9355 & 3.09 & -9.95737 & -16.4994 & -34.4904 \\
\hline J0426+4933 & 0.922475 & -13.4056 & 3.6 & -8.7972 & -14.5997 & -32.6571 \\
\hline J0437-4715 & 0.005757 & -19.2418 & 0.16 & -16.8382 & -18.2312 & -34.9364 \\
\hline $\mathrm{J} 0448-2749$ & 0.450448 & -15.8297 & 2.87 & -11.5326 & -16.7125 & -34.6715 \\
\hline
\end{tabular}


Table 1 continued

\begin{tabular}{|c|c|c|c|c|c|c|}
\hline Pulsar & $P(\mathrm{~s})$ & $\log \dot{P}$ & $d(\mathrm{kpc})$ & $\log \varepsilon$ & $\log \eta$ & $\log h$ \\
\hline J0449-7031 & 0.479164 & -14.4724 & 49.7 & -10.1484 & -15.382 & -34.5794 \\
\hline J0450-1248 & 0.438014 & -15.9872 & 3.27 & -11.7022 & -16.8578 & -34.8734 \\
\hline J0452-1759 & 0.548939 & -14.2403 & 0.4 & -9.85736 & -15.209 & -32.3121 \\
\hline $\mathrm{J} 0453+1559$ & 0.045782 & -17.7305 & 1.83 & -14.4263 & -17.6203 & -35.3838 \\
\hline $\mathrm{J} 0454+5543$ & 0.340729 & -14.6253 & 1.18 & -10.4494 & -15.3868 & -32.9597 \\
\hline J0455-6951 & 0.320423 & -13.9914 & 49.7 & -9.84222 & -14.7263 & -33.9237 \\
\hline J0456-7031 & 0.800132 & -13.4353 & 49.7 & -8.88872 & -14.5676 & -33.7651 \\
\hline J0457-6337 & 2.49701 & -15.6778 & 2.94 & -10.6369 & -17.3043 & -35.2738 \\
\hline $\mathrm{J} 0458-0505$ & 1.88348 & -15.2757 & 3.83 & -10.3573 & -16.7798 & -34.8641 \\
\hline J0459-0210 & 1.13308 & -14.8539 & 1.3 & -10.1562 & -16.1373 & -33.7523 \\
\hline $\mathrm{J} 0501+4516$ & 5.7621 & -11.2351 & 2.2 & -5.83104 & -13.2248 & -31.0683 \\
\hline $\mathrm{J} 0502+4654$ & 0.638565 & -14.2534 & 1.78 & -9.80471 & -15.2877 & -33.0392 \\
\hline $\mathrm{J} 0502-6617$ & 0.691251 & -13.6364 & 49.7 & -9.1533 & -14.7052 & -33.9026 \\
\hline $\mathrm{J} 0511-6508$ & 0.322062 & -15.6861 & 2.17 & -11.5347 & -16.4232 & -34.2607 \\
\hline J0519-6932 & 0.263212 & -15.1568 & 49.7 & -11.093 & -15.8062 & -35.0036 \\
\hline J0520-2553 & 0.241642 & -16.5214 & 3.47 & -12.4948 & -17.1338 & -35.1751 \\
\hline J0522-6847 & 0.674532 & -13.752 & 49.7 & -9.27957 & -14.8102 & -34.0076 \\
\hline $\mathrm{J} 0525+1115$ & 0.354438 & -16.1331 & 7.68 & -11.9401 & -16.9118 & -35.2982 \\
\hline J0529-6652 & 0.975725 & -13.8097 & 49.7 & -9.17689 & -15.0281 & -34.2256 \\
\hline J0532-6639 & 0.642743 & -14.2716 & 49.7 & -9.82016 & -15.3088 & -34.5062 \\
\hline $\mathrm{J} 0533+0402$ & 0.963018 & -15.7959 & 6.66 & -11.1688 & -17.0087 & -35.3332 \\
\hline $\mathrm{J} 0534+2200$ & 0.033392 & -12.3757 & 2 & -9.20862 & -12.1285 & -29.9306 \\
\hline J0534-6703 & 1.81757 & -12.3716 & 49.7 & -7.46867 & -13.8603 & -33.0577 \\
\hline J0535-6935 & 0.200511 & -13.9393 & 49.7 & -9.99371 & -14.4706 & -33.668 \\
\hline J0536-7543 & 1.24586 & -15.248 & 1.04 & -10.509 & -16.5726 & -34.0907 \\
\hline J0537-6910 & 0.016122 & -13.2857 & 49.7 & -10.4348 & -12.7222 & -31.9197 \\
\hline $\mathrm{J} 0538+2817$ & 0.143158 & -14.4353 & 1.3 & -10.6361 & -14.8203 & -32.4353 \\
\hline $\mathrm{J} 0540+3207$ & 0.524271 & -15.3487 & 2.37 & -10.9857 & -16.2974 & -34.1732 \\
\hline J0540-7125 & 1.28601 & -15.0862 & 2.69 & -10.3335 & -16.4246 & -34.3554 \\
\hline $\mathrm{J} 0543+2329$ & 0.245975 & -13.8125 & 3.54 & -9.77814 & -14.4325 & -32.4826 \\
\hline J0543-6851 & 0.708954 & -14.4045 & 49.7 & -9.91043 & -15.4843 & -34.6817 \\
\hline $\mathrm{J} 0546+2441$ & 2.84385 & -14.1163 & 3.16 & -9.01898 & -15.7994 & -33.8001 \\
\hline J0555-7056 & 0.827838 & -14.2248 & 49.7 & -9.66336 & -15.3718 & -34.5693 \\
\hline $\mathrm{J} 0557+1550$ & 0.002556 & -20.1337 & 5.65 & -18.0827 & -18.7704 & -37.0235 \\
\hline J0600-5756 & 2.26137 & -14.556 & 2.55 & -9.55813 & -16.1395 & -34.0471 \\
\hline J0601-0527 & 0.395969 & -14.8861 & 7.54 & -10.6449 & -15.7129 & -34.0913 \\
\hline $\mathrm{J} 0609+2130$ & 0.055698 & -18.6289 & 1.82 & -15.2396 & -18.6039 & -36.3651 \\
\hline J0610-2100 & 0.003861 & -19.9066 & 5.64 & -17.6764 & -18.7224 & -36.9748 \\
\hline $\mathrm{J} 0612+3721$ & 0.297982 & -16.2255 & 1.49 & -12.1078 & -16.9288 & -34.6031 \\
\hline J0613-0200 & 0.003062 & -20.0182 & 1.09 & -17.8887 & -18.7333 & -36.2718 \\
\hline $\mathrm{J} 0613+3731$ & 0.619198 & -14.4895 & 1.05 & -10.0542 & -15.5104 & -33.0327 \\
\hline J0614+2229 & 0.33496 & -13.2262 & 4.74 & -9.05777 & -13.9804 & -32.1572 \\
\hline J0614-3329 & 0.003149 & -19.757 & 1.02 & -17.6153 & -18.4843 & -35.9939 \\
\hline $\mathrm{J} 0621+0336$ & 0.269954 & -17.1403 & 3.58 & -13.0655 & -17.8007 & -35.8556 \\
\hline $\mathrm{J} 0621+1002$ & 0.028854 & -19.3251 & 1.88 & -16.2215 & -19.0145 & -36.7897 \\
\hline $\mathrm{J} 0623+0340$ & 0.61376 & -16.0535 & 2.53 & -11.6221 & -17.0707 & -34.9749 \\
\hline J0624-0424 & 1.03908 & -15.0809 & 4.28 & -10.4208 & -16.3267 & -34.4592 \\
\hline
\end{tabular}


Table 1 continued

\begin{tabular}{|c|c|c|c|c|c|c|}
\hline Pulsar & $P(\mathrm{~s})$ & $\log \dot{P}$ & $d(\mathrm{kpc})$ & $\log \varepsilon$ & $\log \eta$ & $\log h$ \\
\hline J0627+0649 & 0.346523 & -14.7696 & 4 & -10.5864 & -15.5384 & -33.6416 \\
\hline $\mathrm{J} 0627+0706$ & 0.475877 & -13.5258 & 7.88 & -9.20484 & -14.4324 & -32.83 \\
\hline J0628+0909 & 1.24142 & -15.2612 & 4 & -10.5238 & -16.5843 & -34.6874 \\
\hline J0629+2415 & 0.476623 & -14.699 & 4.67 & -10.3773 & -15.6063 & -33.7767 \\
\hline J0630-0046 & 0.680575 & -14.426 & 5.57 & -9.94964 & -15.488 & -33.7349 \\
\hline J0630-2834 & 1.24442 & -14.1475 & 0.32 & -9.4091 & -15.4716 & -32.4778 \\
\hline J0631+1036 & 0.2878 & -12.9788 & 6.54 & -8.87627 & -13.667 & -31.9837 \\
\hline J0633+1746 & 0.237099 & -13.9586 & 0.25 & -9.94022 & -14.5627 & -31.4617 \\
\hline J0633-2015 & 3.25321 & -14.4179 & 7.84 & -9.26217 & -16.1594 & -34.5548 \\
\hline J0636-4549 & 1.9846 & -14.4989 & 0.98 & -9.55782 & -16.0258 & -33.518 \\
\hline $\mathrm{J} 0636+5129$ & 0.002869 & -20.4711 & 0.2 & -18.3699 & -19.158 & -35.9601 \\
\hline $\mathrm{J} 0645+5158$ & 0.008853 & -20.308 & 0.77 & -17.7175 & -19.4843 & -36.8718 \\
\hline J0646+0905 & 0.903913 & -15.1331 & 9.5 & -10.5335 & -16.3184 & -34.7972 \\
\hline $\mathrm{J} 0647+0913$ & 1.23485 & -14.1925 & 10.84 & -9.4574 & -15.5132 & -34.0493 \\
\hline J0652-0142 & 0.924054 & -15.8182 & 5.83 & -11.209 & -17.013 & -35.2797 \\
\hline $\mathrm{J} 0653+8051$ & 1.21444 & -14.4202 & 3.37 & -9.69239 & -15.7337 & -33.7624 \\
\hline J0656-2228 & 1.22475 & -16.5735 & 1.79 & -11.842 & -17.8907 & -35.6446 \\
\hline J0656-5449 & 0.183157 & -16.4962 & 4.88 & -12.5899 & -16.9882 & -35.1777 \\
\hline $\mathrm{J} 0658+0022$ & 0.563295 & -14.0386 & 6.46 & -9.64439 & -15.0185 & -33.3298 \\
\hline J0659+1414 & 0.384891 & -13.2596 & 0.28 & -9.03085 & -14.0741 & -31.0223 \\
\hline $\mathrm{J} 0700+6418$ & 0.195671 & -18.1643 & 0.49 & -14.2293 & -18.685 & -35.8762 \\
\hline J0709-5923 & 0.485268 & -15.8996 & 4.94 & -11.5702 & -16.8148 & -35.0095 \\
\hline $\mathrm{J} 0711+0931$ & 1.21409 & -15.3979 & 2.39 & -10.6702 & -16.7113 & -34.5908 \\
\hline $\mathrm{J} 0711-6830$ & 0.005491 & -19.8268 & 1.04 & -17.4437 & -18.7956 & -36.3137 \\
\hline J0719-2545 & 0.974725 & -14.1379 & 17.33 & -9.50553 & -15.3559 & -34.0958 \\
\hline $\mathrm{J} 0720-3125$ & 8.39112 & -13.1561 & 0.4 & -7.58887 & -15.3091 & -32.4122 \\
\hline J0721-2038 & 0.015542 & -19.3565 & 3.87 & -16.5216 & -18.7772 & -36.866 \\
\hline J0725-1635 & 0.424311 & -16.0334 & 4.68 & -11.7623 & -16.8902 & -35.0615 \\
\hline J0726-2612 & 3.44231 & -12.5331 & 3.01 & -7.35283 & -14.2991 & -32.2788 \\
\hline J0729-1448 & 0.251659 & -12.9469 & 4.37 & -8.90266 & -13.5769 & -31.7184 \\
\hline J0729-1836 & 0.51016 & -13.7212 & 3.25 & -9.37009 & -14.6581 & -32.671 \\
\hline J0733-2345 & 1.79625 & -14.2441 & 11.95 & -9.34631 & -15.7276 & -34.3061 \\
\hline J0737-2202 & 0.320366 & -14.262 & 4.3 & -10.1129 & -14.9968 & -33.1313 \\
\hline J0738-4042 & 0.37492 & -14.7905 & 1.6 & -10.5731 & -15.5936 & -33.2988 \\
\hline J0742-2822 & 0.166762 & -13.7747 & 2 & -9.90914 & -14.2259 & -32.028 \\
\hline J0745-5353 & 0.214836 & -14.5638 & 7.14 & -10.5883 & -15.1251 & -33.4798 \\
\hline J0746-4529 & 2.79102 & -13.7773 & 5.71 & -8.68807 & -15.4522 & -33.7099 \\
\hline J0749-4247 & 1.09545 & -15.0101 & 2.36 & -10.3271 & -16.2788 & -34.1528 \\
\hline $\mathrm{J} 0751+1807$ & 0.003479 & -20.1085 & 0.4 & -17.9236 & -18.8791 & -35.9822 \\
\hline $\mathrm{J} 0754+3231$ & 1.44235 & -14.9666 & 3.92 & -10.1641 & -16.3548 & -34.4491 \\
\hline J0758-1528 & 0.682265 & -14.7905 & 3.72 & -10.3131 & -15.8536 & -33.9252 \\
\hline J0804-3647 & 2.19199 & -14.3757 & 7.79 & -9.39143 & -15.9457 & -34.3383 \\
\hline J0807-5421 & 0.526644 & -15.4225 & 8.76 & -11.0575 & -16.3732 & -34.8167 \\
\hline J0808-3937 & 0.866349 & -15.3969 & 5.62 & -10.8157 & -16.5637 & -34.8145 \\
\hline J0809-4753 & 0.547199 & -14.5114 & 12.71 & -10.1299 & -15.4787 & -34.0839 \\
\hline J0812-3905 & 0.482596 & -16.3478 & 26.83 & -12.0207 & -17.2605 & -36.1902 \\
\hline
\end{tabular}


Table 1 continued

\begin{tabular}{|c|c|c|c|c|c|c|}
\hline Pulsar & $P(\mathrm{~s})$ & $\log \dot{P}$ & $d(\mathrm{kpc})$ & $\log \varepsilon$ & $\log \eta$ & $\log h$ \\
\hline J0814+7429 & 1.29224 & -15.7747 & 0.43 & -11.0199 & -17.1152 & -34.2497 \\
\hline J0815+0939 & 0.645161 & -15.857 & 4.52 & -11.4039 & -16.8958 & -35.052 \\
\hline J0818-3049 & 0.763742 & -15.2366 & 4.17 & -10.7102 & -16.3487 & -34.4699 \\
\hline J0818-3232 & 2.16126 & -15.1232 & 3.82 & -10.145 & -16.6871 & -34.7702 \\
\hline J0820-1350 & 1.23813 & -14.6757 & 1.9 & -9.9395 & -15.9976 & -33.7774 \\
\hline J0820-3826 & 0.124836 & -14.6126 & 6.82 & -10.8728 & -14.9381 & -33.2729 \\
\hline J0820-3921 & 1.07357 & -13.9136 & 6.19 & -9.23936 & -15.1736 & -33.4664 \\
\hline J0820-4114 & 0.545446 & -16.7235 & 2.38 & -12.3433 & -17.6894 & -35.5671 \\
\hline J0821-4221 & 0.396728 & -14.4584 & 18.16 & -10.2165 & -15.2861 & -34.0462 \\
\hline J0821-4300 & 0.112799 & -17.0325 & 2.2 & -13.3367 & -17.3139 & -35.1574 \\
\hline $\mathrm{J} 0823+0159$ & 0.864873 & -15.9788 & 1.44 & -11.3984 & -17.1449 & -34.8043 \\
\hline $\mathrm{J} 0826+2637$ & 0.530661 & -14.767 & 0.32 & -10.3987 & -15.721 & -32.7272 \\
\hline J0828-3417 & 1.84892 & -15.0017 & 0.54 & -10.0914 & -16.4978 & -33.7313 \\
\hline J0831-4406 & 0.311674 & -14.8928 & 12.73 & -10.7556 & -15.6156 & -34.2215 \\
\hline J0834-4159 & 0.121116 & -14.3526 & 9.74 & -10.626 & -14.665 & -33.1546 \\
\hline J0835-3707 & 0.541404 & -14.0097 & 2.34 & -9.63269 & -14.9723 & -32.8426 \\
\hline $\mathrm{J} 0835-4510$ & 0.089328 & -12.9031 & 0.28 & -9.30865 & -13.0832 & -30.0314 \\
\hline $\mathrm{J} 0837+0610$ & 1.27377 & -14.1675 & 0.76 & -9.41895 & -15.5017 & -32.8836 \\
\hline J0837-4135 & 0.751624 & -14.451 & 1.5 & -9.93154 & -15.5561 & -33.2333 \\
\hline J0838-2621 & 0.308581 & -16.4089 & 4.56 & -12.2761 & -17.1275 & -35.2875 \\
\hline J0838-3947 & 1.70395 & -15.0899 & 8.21 & -10.215 & -16.5505 & -34.9659 \\
\hline J0840-5332 & 0.720612 & -14.7852 & 7.77 & -10.284 & -15.872 & -34.2635 \\
\hline J0842-4851 & 0.644354 & -14.0223 & 8.68 & -9.5697 & -15.0605 & -33.5001 \\
\hline J0843-5022 & 0.208956 & -15.7645 & 7.69 & -11.801 & -16.3137 & -34.7007 \\
\hline J0846-3533 & 1.1161 & -14.7959 & 1.43 & -10.1047 & -16.0727 & -33.7291 \\
\hline $\mathrm{J} 0847-4316$ & 5.97749 & -12.9208 & 13.44 & -7.50085 & -14.9265 & -33.5559 \\
\hline J0849-6322 & 0.367953 & -15.1018 & 8.35 & -10.8926 & -15.8968 & -34.3195 \\
\hline J0849+8028 & 1.60223 & -15.3507 & 3.38 & -10.5025 & -16.7845 & -34.8145 \\
\hline J0855-3331 & 1.26754 & -14.1993 & 1.21 & -9.45287 & -15.5314 & -33.1152 \\
\hline J0855-4644 & 0.064686 & -14.1391 & 9.9 & -10.6848 & -14.179 & -32.6757 \\
\hline J0855-4658 & 0.575072 & -13.8665 & 28.35 & -9.46329 & -14.8553 & -33.8089 \\
\hline J0856-6137 & 0.962509 & -14.7747 & 6.53 & -10.1478 & -15.9872 & -34.3032 \\
\hline J0857-4424 & 0.326774 & -13.6326 & 6.53 & -9.47494 & -14.376 & -32.692 \\
\hline J0900-3144 & 0.01111 & -19.3089 & 0.82 & -16.6198 & -18.5838 & -35.9987 \\
\hline J0901-4624 & 0.441995 & -13.058 & 7.46 & -8.76912 & -13.9326 & -32.3064 \\
\hline J0902-6325 & 0.660313 & -15.9706 & 4.44 & -11.5074 & -17.0195 & -35.168 \\
\hline J0904-4246 & 0.965171 & -14.7235 & 4.37 & -10.0955 & -15.9373 & -34.0788 \\
\hline J0904-7459 & 0.549553 & -15.3344 & 4.41 & -10.951 & -16.3036 & -34.4491 \\
\hline J0905-4536 & 0.988281 & -15.8268 & 6.35 & -11.1885 & -17.0508 & -35.3547 \\
\hline J0905-5127 & 0.346287 & -13.6038 & 8.38 & -9.42091 & -14.3724 & -32.7967 \\
\hline J0905-6019 & 0.340854 & -15.2823 & 4.4 & -11.1063 & -16.044 & -34.1886 \\
\hline J0907-5157 & 0.253556 & -14.7375 & 2.63 & -10.69 & -15.3708 & -33.2918 \\
\hline J0908-1739 & 0.401626 & -15.1746 & 1.01 & -10.9273 & -16.0075 & -33.5129 \\
\hline J0908-4913 & 0.106755 & -13.8182 & 1 & -10.1463 & -14.0757 & -31.5768 \\
\hline J0909-7212 & 1.36289 & -15.4776 & 4 & -10.6996 & -16.8412 & -34.9443 \\
\hline J0912-3851 & 1.52608 & -14.4449 & 0.62 & -9.61788 & -15.8576 & -33.1511 \\
\hline J0919-6040 & 1.21698 & -17 & 3.54 & -12.2713 & -18.3144 & -36.3645 \\
\hline
\end{tabular}


Table 1 continued

\begin{tabular}{|c|c|c|c|c|c|c|}
\hline Pulsar & $P(\mathrm{~s})$ & $\log \dot{P}$ & $d(\mathrm{kpc})$ & $\log \varepsilon$ & $\log \eta$ & $\log h$ \\
\hline $\mathrm{J} 0921+6254$ & 1.56799 & -14.4425 & 0.79 & -9.6037 & -15.867 & -33.2657 \\
\hline J0922+0638 & 0.430627 & -13.8633 & 1.1 & -9.58573 & -14.7265 & -32.269 \\
\hline J0922-4949 & 0.950289 & -13.0106 & 10.37 & -8.38924 & -14.2176 & -32.7344 \\
\hline J0924-5302 & 0.746295 & -13.4498 & 5.61 & -8.93341 & -14.5518 & -32.8019 \\
\hline J0924-5814 & 0.739501 & -14.3116 & 1.87 & -9.79919 & -15.4097 & -33.1826 \\
\hline J0931-1902 & 0.004638 & -20.3883 & 3.63 & -18.0785 & -19.2838 & -37.3447 \\
\hline J0932-3217 & 1.93163 & -15.6021 & 3.84 & -10.6727 & -17.1171 & -35.2025 \\
\hline J0932-5327 & 4.39216 & -14.0773 & 3.91 & -8.79114 & -15.9491 & -34.0423 \\
\hline J0934-4154 & 0.570409 & -15.5702 & 3.2 & -11.1706 & -16.5556 & -34.5618 \\
\hline J0934-5249 & 1.44477 & -14.3325 & 2.93 & -9.5293 & -15.7215 & -33.6894 \\
\hline J0940-5428 & 0.087545 & -13.4828 & 4.27 & -9.89712 & -13.6542 & -31.7857 \\
\hline J0941-5244 & 0.658558 & -14.9431 & 5.64 & -10.481 & -15.9908 & -34.2432 \\
\hline J0942-5552 & 0.664367 & -13.6402 & 0.3 & -9.1743 & -14.6917 & -31.6699 \\
\hline J0942-5657 & 0.808127 & -13.4023 & 5.02 & -8.85137 & -14.5389 & -32.7407 \\
\hline $\mathrm{J} 0943+1631$ & 1.08742 & -16.0405 & 1.76 & -11.3606 & -17.306 & -35.0526 \\
\hline J0944-1354 & 0.570264 & -16.3439 & 0.69 & -11.9444 & -17.3291 & -34.669 \\
\hline J0945-4833 & 0.331586 & -14.3161 & 2.71 & -10.152 & -15.0658 & -32.9998 \\
\hline J0946+0951 & 1.09771 & -14.4572 & 0.96 & -9.77323 & -15.7268 & -33.2101 \\
\hline J0949-6902 & 0.640016 & -15.1959 & 8.68 & -10.7462 & -16.2312 & -34.6708 \\
\hline J0952-3839 & 1.37382 & -15.2366 & 8.44 & -10.4552 & -16.6036 & -35.0311 \\
\hline J0953+0755 & 0.253065 & -15.6383 & 0.26 & -11.5916 & -16.2707 & -33.1867 \\
\hline J0954-5430 & 0.472834 & -13.3575 & 6.2 & -9.03937 & -14.2614 & -32.5548 \\
\hline J0955-5304 & 0.862118 & -14.4535 & 4.87 & -9.87444 & -15.6182 & -33.8068 \\
\hline J0957-5432 & 0.203557 & -14.71 & 7 & -10.7578 & -15.2478 & -33.594 \\
\hline J0959-4809 & 0.670086 & -16.0862 & 2.96 & -11.6166 & -17.1415 & -35.1138 \\
\hline J1000-5149 & 0.255677 & -15.0182 & 2.33 & -10.967 & -15.655 & -33.5234 \\
\hline $\mathrm{J} 1001-5507$ & 1.43658 & -13.289 & 0.3 & -8.48825 & -14.6755 & -31.6537 \\
\hline J1001-5559 & 1.66118 & -15.0655 & 3.94 & -10.2016 & -16.5151 & -34.6116 \\
\hline J1001-5939 & 7.73364 & -13.2226 & 3.3 & -7.69074 & -15.3401 & -33.3597 \\
\hline J1002-5559 & 0.777501 & -14.8041 & 17.42 & -10.2699 & -15.9239 & -34.6661 \\
\hline J1002-5919 & 0.713489 & -15.983 & 15.13 & -11.4861 & -17.0655 & -35.7464 \\
\hline J1003-4747 & 0.307072 & -13.6556 & 3.44 & -9.52491 & -14.372 & -32.4096 \\
\hline J1006-6311 & 0.835797 & -15.2418 & 9.3 & -10.6763 & -16.3931 & -34.8626 \\
\hline $\mathrm{J} 1012-2337$ & 2.51795 & -15.055 & 1.29 & -10.0105 & -16.6852 & -34.2969 \\
\hline $\mathrm{J} 1012+5307$ & 0.005256 & -19.767 & 0.7 & -17.4029 & -18.7168 & -36.063 \\
\hline $\mathrm{J} 1012-5830$ & 2.13359 & -13.4237 & 5.34 & -8.4511 & -14.9819 & -33.2105 \\
\hline J1012-5857 & 0.819911 & -13.752 & 10.14 & -9.19481 & -14.8949 & -33.402 \\
\hline J1013-5934 & 0.442901 & -15.2534 & 11.32 & -10.9636 & -16.1288 & -34.6837 \\
\hline J1015-5719 & 0.139882 & -13.2411 & 4.87 & -9.45187 & -13.616 & -31.8046 \\
\hline J1016-5345 & 0.769584 & -14.7144 & 2.5 & -10.1847 & -15.8298 & -33.7288 \\
\hline J1016-5819 & 0.087834 & -15.1561 & 4.62 & -11.569 & -15.329 & -33.4947 \\
\hline J1016-5857 & 0.107386 & -13.0926 & 9.31 & -9.41819 & -13.3527 & -31.8227 \\
\hline $\mathrm{J} 1017-5621$ & 0.503459 & -14.5045 & 11.78 & -10.159 & -15.4356 & -34.0078 \\
\hline J1017-7156 & 0.002339 & -20.6536 & 0.26 & -18.6412 & -19.2518 & -36.1679 \\
\hline $\mathrm{J} 1018-1642$ & 1.80469 & -14.7595 & 3.27 & -9.8596 & -16.245 & -34.2606 \\
\hline J1019-5749 & 0.162499 & -13.6968 & 30 & -9.8425 & -14.1368 & -33.115 \\
\hline
\end{tabular}


Table 1 continued

\begin{tabular}{|c|c|c|c|c|c|c|}
\hline Pulsar & $P(\mathrm{~s})$ & $\log \dot{P}$ & $d(\mathrm{kpc})$ & $\log \varepsilon$ & $\log \eta$ & $\log h$ \\
\hline J1020-5921 & 1.2383 & -13.3925 & 2.62 & -8.65627 & -14.7145 & -32.6339 \\
\hline J1020-6026 & 0.14048 & -14.1713 & 29.58 & -10.3803 & -14.5481 & -33.5202 \\
\hline $\mathrm{J} 1021-5601$ & 0.670026 & -16.2725 & 4.18 & -11.8029 & -17.3277 & -35.4499 \\
\hline $\mathrm{J} 1022+1001$ & 0.016453 & -19.3635 & 0.74 & -16.5038 & -18.8089 & -36.1792 \\
\hline $\mathrm{J} 1022-5813$ & 1.64373 & -12.8386 & 30 & -7.97935 & -14.2836 & -33.2618 \\
\hline $\mathrm{J} 1023+0038$ & 0.001688 & -19.9208 & 1.37 & -18.05 & -18.3773 & -36.0151 \\
\hline J1024-0719 & 0.005162 & -19.7328 & 1.1 & -17.3766 & -18.6748 & -36.2172 \\
\hline J1028-5819 & 0.091403 & -13.7932 & 2.76 & -10.1888 & -13.9833 & -31.9253 \\
\hline $\mathrm{J} 1031-6117$ & 0.306411 & -14.7496 & 30 & -10.6198 & -15.465 & -34.4432 \\
\hline J1032-5206 & 2.40762 & -13.7471 & 4.33 & -8.72211 & -15.3579 & -33.4954 \\
\hline J1032-5911 & 0.464208 & -14.5229 & 7.26 & -10.2127 & -15.4187 & -33.7807 \\
\hline $\mathrm{J} 1034-3224$ & 1.15059 & -15.6383 & 4.68 & -10.9339 & -16.9283 & -35.0996 \\
\hline $\mathrm{J} 1035-6345$ & 0.579577 & -15.4559 & 6.54 & -11.0494 & -16.4482 & -34.7648 \\
\hline J1036-4926 & 0.510368 & -14.7825 & 8.71 & -10.4312 & -15.7195 & -34.1606 \\
\hline J1036-6559 & 0.533502 & -14.8665 & 7.86 & -10.4959 & -15.8227 & -34.2192 \\
\hline $\mathrm{J} 1038+0032$ & 0.028852 & -19.1739 & 2.36 & -16.0703 & -18.8632 & -36.7372 \\
\hline $\mathrm{J} 1038-5831$ & 0.661992 & -14.9031 & 2.43 & -10.4388 & -15.9531 & -33.8398 \\
\hline J1041-1942 & 1.38637 & -15.0246 & 3.18 & -10.2392 & -16.3956 & -34.3991 \\
\hline $\mathrm{J} 1042-5521$ & 1.17086 & -14.172 & 6.98 & -9.46003 & -15.4696 & -33.8146 \\
\hline J1043-6116 & 0.288602 & -13.983 & 18.2 & -9.87921 & -14.6724 & -33.4335 \\
\hline J1045-4509 & 0.007474 & -19.752 & 0.34 & -17.235 & -18.8547 & -35.8873 \\
\hline $\mathrm{J} 1046+0304$ & 0.326271 & -15.9066 & 2.25 & -11.7495 & -16.6493 & -34.5025 \\
\hline J1046-5813 & 0.369427 & -14.9431 & 4.8 & -10.7321 & -15.7398 & -33.9221 \\
\hline $\mathrm{J} 1047-3032$ & 0.330328 & -16.2147 & 4.14 & -12.0523 & -16.9628 & -35.0808 \\
\hline J1047-6709 & 0.198451 & -14.7721 & 4.67 & -10.831 & -15.2989 & -33.4693 \\
\hline $\mathrm{J} 1048-5832$ & 0.123671 & -13.0164 & 2.9 & -9.28065 & -13.3378 & -31.3012 \\
\hline J1049-5833 & 2.20233 & -14.3556 & 9.73 & -9.36923 & -15.9276 & -34.4168 \\
\hline J1050-5953 & 6.45208 & -10.4191 & 9 & -4.96592 & -12.4579 & -30.9132 \\
\hline J1052-5954 & 0.180592 & -13.699 & 13.55 & -9.79882 & -14.1848 & -32.8178 \\
\hline $\mathrm{J} 1052-6348$ & 0.38383 & -15.4123 & 5.27 & -11.1847 & -16.2256 & -34.4484 \\
\hline J1054-5943 & 0.346909 & -14.3904 & 6.81 & -10.2067 & -15.1598 & -33.494 \\
\hline J1054-5946 & 0.228324 & -15.6799 & 5.81 & -11.6778 & -16.2676 & -34.5328 \\
\hline $\mathrm{J} 1054-6452$ & 1.84 & -14.5031 & 13.53 & -9.5948 & -15.997 & -34.6294 \\
\hline $\mathrm{J} 1055-6022$ & 0.947558 & -13.0343 & 25.63 & -8.41427 & -14.2401 & -33.1499 \\
\hline $\mathrm{J} 1055-6028$ & 0.099661 & -13.5302 & 30 & -9.8882 & -13.7579 & -32.736 \\
\hline $\mathrm{J} 1055-6236$ & 0.448635 & -15.15 & 3.64 & -10.8546 & -16.031 & -34.0932 \\
\hline J1055-6905 & 2.9194 & -13.6925 & 11.91 & -8.58376 & -15.3869 & -33.9639 \\
\hline J1056-5709 & 0.676082 & -15.2396 & 17.61 & -10.7661 & -16.2987 & -35.0455 \\
\hline $\mathrm{J} 1056-6258$ & 0.422447 & -14.4461 & 2.4 & -10.1769 & -15.301 & -33.1823 \\
\hline J1056-7117 & 0.02631 & -19.2007 & 5.27 & -16.1371 & -18.8499 & -37.0728 \\
\hline J1057-4754 & 0.628306 & -15.6478 & 3.03 & -11.2062 & -16.6751 & -34.6576 \\
\hline $\mathrm{J} 1057-5226$ & 0.197108 & -14.2343 & 1.53 & -10.2962 & -14.7582 & -32.4439 \\
\hline J1057-7914 & 1.3474 & -14.8761 & 3.46 & -10.1032 & -16.2348 & -34.2749 \\
\hline J1058-5957 & 0.61627 & -15.1824 & 7.11 & -10.7492 & -16.2014 & -34.5543 \\
\hline $\mathrm{J} 1059-5742$ & 1.185 & -14.3665 & 2.75 & -9.64936 & -15.6694 & -33.6098 \\
\hline
\end{tabular}


Table 1 continued

\begin{tabular}{|c|c|c|c|c|c|c|}
\hline Pulsar & $P(\mathrm{~s})$ & $\log \dot{P}$ & $d(\mathrm{kpc})$ & $\log \varepsilon$ & $\log \eta$ & $\log h$ \\
\hline $\mathrm{J} 1101-6101$ & 0.0628 & -14.0675 & 7 & -10.6261 & -14.0946 & -32.4408 \\
\hline J1101-6424 & 0.005109 & -20.7447 & 7.86 & -18.3929 & -19.6822 & -38.0787 \\
\hline J1103-5403 & 0.003393 & -20.4342 & 3.16 & -18.2601 & -19.1939 & -37.1946 \\
\hline $\mathrm{J} 1103-6025$ & 0.396587 & -15.0044 & 6.83 & -10.7626 & -15.8319 & -34.1673 \\
\hline J1104-6103 & 0.280905 & -14.7055 & 2.31 & -10.6135 & -15.3832 & -33.2479 \\
\hline J1105-6107 & 0.063193 & -13.8013 & 7.07 & -10.3572 & -13.8312 & -32.1816 \\
\hline J1106-6438 & 2.71793 & -14.6326 & 7.88 & -9.55495 & -16.296 & -34.6936 \\
\hline J1107-5907 & 0.252773 & -17.0453 & 1.81 & -12.9991 & -17.6772 & -35.4359 \\
\hline J1107-5947 & 1.51653 & -15.4685 & 3.52 & -10.6442 & -16.8785 & -34.9261 \\
\hline J1107-6143 & 1.7994 & -12.8069 & 13.72 & -7.9083 & -14.2912 & -32.9296 \\
\hline J1110-5637 & 0.558253 & -14.6861 & 9.18 & -10.2958 & -15.6621 & -34.126 \\
\hline $\mathrm{J} 1112-6103$ & 0.064962 & -13.5017 & 30 & -10.0456 & -13.5435 & -32.5217 \\
\hline J1112-6613 & 0.334213 & -15.0841 & 19.31 & -10.9166 & -15.8372 & -34.6241 \\
\hline J1112-6926 & 0.820484 & -14.5467 & 12.32 & -9.98916 & -15.6899 & -34.2816 \\
\hline $\mathrm{J} 1114-6100$ & 0.88082 & -13.3363 & 30 & -8.74796 & -14.5103 & -33.4885 \\
\hline $\mathrm{J} 1115+5030$ & 1.65644 & -14.6038 & 0.51 & -9.74117 & -16.0521 & -33.2608 \\
\hline $\mathrm{J} 1115-6052$ & 0.259777 & -14.1409 & 6.76 & -10.0828 & -14.7846 & -33.1156 \\
\hline J1116-4122 & 0.943158 & -14.0996 & 2.68 & -9.4816 & -15.3034 & -33.2326 \\
\hline J1117-6154 & 0.505097 & -13.9031 & 22.38 & -9.55626 & -14.8356 & -33.6865 \\
\hline J1117-6447 & 1.15527 & -15.6198 & 27.12 & -10.9137 & -16.9116 & -35.846 \\
\hline J1119-6127 & 0.407963 & -11.3958 & 8.4 & -7.1417 & -12.2355 & -30.6609 \\
\hline J1119-7936 & 2.2806 & -14.4353 & 1.52 & -9.43383 & -16.0225 & -33.7054 \\
\hline $\mathrm{J} 1121-5444$ & 0.535783 & -14.5575 & 15.43 & -10.1851 & -15.5157 & -34.2051 \\
\hline $\mathrm{J} 1123-4844$ & 0.244838 & -16.1844 & 8.74 & -12.1521 & -16.8024 & -35.245 \\
\hline $\mathrm{J} 1123-6102$ & 0.640234 & -14.1898 & 14.75 & -9.73998 & -15.2253 & -33.8951 \\
\hline $\mathrm{J} 1123-6259$ & 0.271434 & -14.2798 & 7.54 & -10.2027 & -14.9427 & -33.3211 \\
\hline J1123-6651 & 0.232976 & -14.5607 & 3.48 & -10.5499 & -15.1571 & -33.1998 \\
\hline J1124-5638 & 0.18556 & -17.0458 & 23.74 & -13.1338 & -17.5434 & -36.4199 \\
\hline J1124-5916 & 0.135477 & -12.1232 & 5 & -8.34789 & -12.4842 & -30.6842 \\
\hline J1124-6421 & 0.479099 & -15.2076 & 14.9 & -10.8837 & -16.1172 & -34.7914 \\
\hline $\mathrm{J} 1125-5825$ & 0.003102 & -19.2154 & 2.98 & -17.0803 & -17.9362 & -35.9114 \\
\hline $\mathrm{J} 1125-6014$ & 0.00263 & -20.3969 & 1.94 & -18.3334 & -19.046 & -36.8348 \\
\hline J1126-6054 & 0.202737 & -15.5513 & 8.05 & -11.6009 & -16.0874 & -34.4942 \\
\hline J1126-6942 & 0.579416 & -14.4815 & 2.07 & -10.075 & -15.4736 & -33.2907 \\
\hline J1128-6219 & 0.515984 & -16.9586 & 30 & -12.6025 & -17.9004 & -36.8786 \\
\hline $\mathrm{J} 1130-5826$ & 0.162323 & -16.7986 & 9.45 & -12.9448 & -17.2381 & -35.7146 \\
\hline J1130-5925 & 0.680984 & -15.0214 & 8.28 & -10.5448 & -16.0836 & -34.5027 \\
\hline J1130-6807 & 0.256353 & -15.3372 & 7.14 & -11.285 & -15.9752 & -34.33 \\
\hline J1132-5627 & 0.175166 & -16.1675 & 21.3 & -12.2806 & -16.6401 & -35.4695 \\
\hline $\mathrm{J} 1133-6250$ & 1.02287 & -15.3449 & 30 & -10.6916 & -16.5838 & -35.562 \\
\hline $\mathrm{J} 1136+1551$ & 1.18791 & -14.4283 & 0.35 & -9.71005 & -15.7322 & -32.7773 \\
\hline J1136-5525 & 0.364706 & -14.0851 & 2.6 & -9.87973 & -14.8762 & -32.7923 \\
\hline $\mathrm{J} 1137-6700$ & 0.556216 & -16.1445 & 19.53 & -11.7558 & -17.1189 & -35.9106 \\
\hline J1138-6207 & 0.117564 & -13.9031 & 24.5 & -10.1894 & -14.2025 & -33.0927 \\
\hline J1141-3107 & 0.538432 & -14.7077 & 2.72 & -10.3332 & -15.668 & -33.6037 \\
\hline $\mathrm{J} 1141-3322$ & 0.291468 & -15.3344 & 3.84 & -11.2264 & -16.0282 & -34.1135 \\
\hline
\end{tabular}


Table 1 continued

\begin{tabular}{|c|c|c|c|c|c|c|}
\hline Pulsar & $P(\mathrm{~s})$ & $\log \dot{P}$ & $d(\mathrm{kpc})$ & $\log \varepsilon$ & $\log \eta$ & $\log h$ \\
\hline $\mathrm{J} 1141-6545$ & 0.393899 & -14.3655 & 3 & -10.1267 & -15.1901 & -33.1682 \\
\hline $\mathrm{J} 1142-6230$ & 0.558383 & -16.1085 & 10.81 & -11.7181 & -17.0845 & -35.6194 \\
\hline $\mathrm{J} 1143-5158$ & 0.675646 & -15.158 & 10.68 & -10.6848 & -16.2169 & -34.7465 \\
\hline J1143-5536 & 0.685358 & -15.3143 & 11.44 & -10.8349 & -16.3793 & -34.9388 \\
\hline J1144-6217 & 0.850665 & -13.5114 & 8.88 & -8.93824 & -14.6704 & -33.1198 \\
\hline J1146-6030 & 0.273372 & -14.7471 & 2.59 & -10.6669 & -15.413 & -33.3274 \\
\hline J1148-5725 & 3.55994 & -13.9547 & 6.99 & -8.75978 & -15.7353 & -34.0808 \\
\hline J1148-6415 & 3.24103 & -14.5686 & 9.07 & -9.4145 & -16.3085 & -34.7671 \\
\hline $\mathrm{J} 1151-6108$ & 0.101633 & -13.9872 & 7.65 & -10.3367 & -14.2233 & -32.6081 \\
\hline $\mathrm{J} 1152-5800$ & 1.78983 & -14.8928 & 7.87 & -9.99653 & -16.3747 & -34.7718 \\
\hline $\mathrm{J} 1152-6012$ & 0.37657 & -14.1746 & 2.09 & -9.95528 & -14.9796 & -32.8008 \\
\hline $\mathrm{J} 1154-6250$ & 0.282012 & -15.2526 & 2.06 & -11.1589 & -15.932 & -33.7469 \\
\hline J1156-5707 & 0.288409 & -13.5768 & 20.4 & -9.47329 & -14.2659 & -33.0766 \\
\hline J1156-5909 & 1.03793 & -14.9914 & 8.76 & -10.3318 & -16.2367 & -34.6803 \\
\hline J1157-5112 & 0.043589 & -18.8447 & 1.88 & -15.5618 & -18.7132 & -36.4884 \\
\hline J1157-6224 & 0.400522 & -14.4056 & 4 & -10.1595 & -15.2374 & -33.3405 \\
\hline J1159-6409 & 0.667486 & -16.6198 & 6.03 & -12.1519 & -17.6734 & -35.9548 \\
\hline J1159-7910 & 0.525074 & -14.5498 & 4.36 & -10.1861 & -15.4991 & -33.6397 \\
\hline J1201-6306 & 0.592136 & -14.4486 & 30 & -10.0327 & -15.4501 & -34.4283 \\
\hline J1202-5820 & 0.452801 & -14.6716 & 4.94 & -10.3723 & -15.5567 & -33.7515 \\
\hline J1204-6843 & 0.308861 & -15.6635 & 5.66 & -11.5303 & -16.3825 & -34.6363 \\
\hline $\mathrm{J} 1210-5226$ & 0.424131 & -16.6536 & 2.6 & -12.3827 & -17.5103 & -35.4263 \\
\hline J1210-5559 & 0.279767 & -15.1391 & 11.65 & -11.0488 & -15.815 & -34.3824 \\
\hline $\mathrm{J} 1210-6550$ & 4.23701 & -15.3655 & 1.58 & -10.095 & -17.2217 & -34.9214 \\
\hline $\mathrm{J} 1211-6324$ & 0.433084 & -15.5901 & 12.3 & -11.31 & -16.4558 & -35.0468 \\
\hline $\mathrm{J} 1214-5830$ & 0.909823 & -16.2725 & 4.83 & -11.67 & -17.4606 & -35.6456 \\
\hline $\mathrm{J} 1215-5328$ & 0.636414 & -15.9393 & 11.24 & -11.4921 & -16.9722 & -35.524 \\
\hline $\mathrm{J} 1216-6223$ & 0.374047 & -13.7747 & 30 & -9.55831 & -14.5768 & -33.5549 \\
\hline J1216-6410 & 0.003539 & -20.7905 & 1.71 & -18.5982 & -19.5685 & -37.3026 \\
\hline $\mathrm{J} 1220-6318$ & 0.789212 & -16.0953 & 13.96 & -11.5546 & -17.2216 & -35.8676 \\
\hline $\mathrm{J} 1222-5738$ & 1.08116 & -15.8239 & 2.12 & -11.1466 & -17.0869 & -34.9143 \\
\hline $\mathrm{J} 1224-6208$ & 0.585761 & -13.6946 & 23.64 & -9.28348 & -14.6915 & -33.5662 \\
\hline J1224-6407 & 0.216476 & -14.3054 & 4 & -10.3265 & -14.87 & -32.9731 \\
\hline $\mathrm{J} 1225-5556$ & 1.01845 & -14.6861 & 5.39 & -10.0347 & -15.9232 & -34.1559 \\
\hline $\mathrm{J} 1225-6035$ & 0.626324 & -15.5406 & 6.19 & -11.1004 & -16.5666 & -34.8593 \\
\hline $\mathrm{J} 1225-6408$ & 0.419618 & -15.0241 & 24.6 & -10.7578 & -15.8761 & -34.7681 \\
\hline $\mathrm{J} 1227-4853$ & 0.001686 & -19.9547 & 2 & -18.0844 & -18.4107 & -36.2128 \\
\hline $\mathrm{J} 1227-6208$ & 0.034528 & -18.7282 & 15.82 & -15.5465 & -18.4955 & -37.1957 \\
\hline $\mathrm{J} 1231-1411$ & 0.003684 & -19.6421 & 0.45 & -17.4323 & -18.4375 & -35.5918 \\
\hline $\mathrm{J} 1231-4609$ & 0.877239 & -16.4202 & 6.16 & -11.8336 & -17.5925 & -35.8831 \\
\hline $\mathrm{J} 1231-6303$ & 1.35124 & -14.8539 & 12.12 & -10.0797 & -16.2138 & -34.7983 \\
\hline $\mathrm{J} 1232-4742$ & 1.87299 & -16.8539 & 1.4 & -11.9379 & -18.3556 & -36.0027 \\
\hline $\mathrm{J} 1232-6501$ & 0.088282 & -18.091 & 10 & -14.5017 & -18.266 & -36.7671 \\
\hline $\mathrm{J} 1233-6312$ & 0.564759 & -14.1599 & 20.49 & -9.76458 & -15.1409 & -33.9535 \\
\hline $\mathrm{J} 1233-6344$ & 0.756892 & -14.4112 & 30 & -9.88868 & -15.5194 & -34.4975 \\
\hline $\mathrm{J} 1235-6354$ & 0.256778 & -15.7055 & 27.05 & -11.6525 & -16.3442 & -35.2775 \\
\hline J1236-5033 & 0.29476 & -15.8069 & 8.29 & -11.694 & -16.5055 & -34.9251 \\
\hline
\end{tabular}


Table 1 continued

\begin{tabular}{|c|c|c|c|c|c|c|}
\hline Pulsar & $P(\mathrm{~s})$ & $\log \dot{P}$ & $d(\mathrm{kpc})$ & $\log \varepsilon$ & $\log \eta$ & $\log h$ \\
\hline $\mathrm{J} 1237-6725$ & 2.11097 & -14.6596 & 7.8 & -9.69162 & -16.2132 & -34.6063 \\
\hline $\mathrm{J} 1238+21$ & 1.11859 & -14.8386 & 1.77 & -10.1465 & -16.1165 & -33.8655 \\
\hline $\mathrm{J} 1239+2453$ & 1.38245 & -15.0177 & 0.84 & -10.2336 & -16.3875 & -33.8129 \\
\hline $\mathrm{J} 1239-6832$ & 1.30191 & -13.9245 & 2.89 & -9.16642 & -15.2682 & -33.2301 \\
\hline $\mathrm{J} 1240-4124$ & 0.512242 & -14.7595 & 3.59 & -10.4065 & -15.6981 & -33.7542 \\
\hline $\mathrm{J} 1243-5735$ & 0.471225 & -16.0969 & 19.2 & -11.7802 & -16.9993 & -35.7836 \\
\hline $\mathrm{J} 1243-6423$ & 0.388481 & -14.3468 & 2 & -10.114 & -15.1653 & -32.9674 \\
\hline $\mathrm{J} 1244-5053$ & 0.275207 & -15 & 8.49 & -10.9169 & -15.6688 & -34.0988 \\
\hline J1244-6359 & 0.147274 & -17.3565 & 12.05 & -13.545 & -17.7538 & -36.3359 \\
\hline $\mathrm{J} 1244-6531$ & 1.54682 & -14.1439 & 30 & -9.31098 & -15.5625 & -34.5406 \\
\hline $\mathrm{J} 1245-6238$ & 2.28309 & -13.9626 & 14.63 & -8.9606 & -15.5502 & -34.2165 \\
\hline J1248-6344 & 0.198335 & -13.7721 & 23.99 & -9.83126 & -14.2987 & -33.1797 \\
\hline $\mathrm{J} 1248-6444$ & 1.23489 & -14.7144 & 9.55 & -9.97936 & -16.0352 & -34.5163 \\
\hline J1249-6507 & 0.434445 & -16.9172 & 8.51 & -12.6358 & -17.7843 & -36.2153 \\
\hline $\mathrm{J} 1251-7407$ & 0.327058 & -15.4377 & 5.22 & -11.2796 & -16.1815 & -34.4002 \\
\hline J1252-6314 & 0.823339 & -15.9666 & 11 & -11.4075 & -17.1113 & -35.6538 \\
\hline $\mathrm{J} 1253-5820$ & 0.255496 & -14.6778 & 2.94 & -10.6269 & -15.3143 & -33.2837 \\
\hline J1254-6150 & 0.184502 & -15.2048 & 2.24 & -11.2954 & -15.7 & -33.5513 \\
\hline $\mathrm{J} 1255-6131$ & 0.657974 & -14.3979 & 7.36 & -9.93628 & -15.4453 & -33.8132 \\
\hline J1257-1027 & 0.617308 & -15.4401 & 2.22 & -11.0061 & -16.4597 & -34.3072 \\
\hline$J 1259-6741$ & 0.663329 & -14.9172 & 2.72 & -10.452 & -15.9681 & -33.9037 \\
\hline $\mathrm{J} 1300+1240$ & 0.006219 & -18.9431 & 0.6 & -16.5059 & -17.966 & -35.2452 \\
\hline J1301-6305 & 0.184528 & -12.5735 & 15.84 & -8.66397 & -13.0687 & -31.7695 \\
\hline $\mathrm{J} 1301-6310$ & 0.66383 & -13.2487 & 2.06 & -8.78321 & -14.2999 & -32.1149 \\
\hline $\mathrm{J} 1302-6313$ & 0.967846 & -14.1986 & 28.06 & -9.56934 & -15.4136 & -34.3627 \\
\hline $\mathrm{J} 1302-6350$ & 0.047763 & -14.6421 & 2.3 & -11.3195 & -14.5503 & -32.4131 \\
\hline $\mathrm{J} 1303-6305$ & 2.30664 & -14.6615 & 13.62 & -9.65511 & -16.2537 & -34.8889 \\
\hline J1305-6203 & 0.427762 & -13.4935 & 24.07 & -9.21884 & -14.3538 & -33.2364 \\
\hline J1305-6256 & 0.478231 & -14.6757 & 30 & -10.3526 & -15.5845 & -34.5627 \\
\hline J1305-6455 & 0.571647 & -14.3947 & 30 & -9.99411 & -15.381 & -34.3592 \\
\hline J1306-6242 & 0.981902 & -14.2321 & 22.63 & -9.59658 & -15.4533 & -34.3091 \\
\hline J1306-6617 & 0.473026 & -14.224 & 29.16 & -9.90569 & -15.1281 & -34.0939 \\
\hline $\mathrm{J} 1307-6318$ & 4.96243 & -13.6757 & 14.45 & -8.33657 & -15.6006 & -34.2615 \\
\hline $\mathrm{J} 1308-4650$ & 1.05883 & -15.279 & 4.91 & -10.6107 & -16.533 & -34.7251 \\
\hline J1308-5844 & 0.4647 & -14.0635 & 8.86 & -9.75286 & -14.9598 & -33.4083 \\
\hline J1309-6415 & 0.619454 & -14.056 & 30 & -9.62055 & -15.0772 & -34.0553 \\
\hline$J 1309-6526$ & 0.398292 & -16.7352 & 19.94 & -12.4915 & -17.5645 & -36.3653 \\
\hline $\mathrm{J} 1311-1228$ & 0.447518 & -15.821 & 2.3 & -11.5268 & -16.701 & -34.5638 \\
\hline $\mathrm{J} 1311-3430$ & 0.00256 & -19.6778 & 3.72 & -17.6261 & -18.3152 & -36.3868 \\
\hline $\mathrm{J} 1312-5402$ & 0.728154 & -15.8297 & 9.65 & -11.3241 & -16.9211 & -35.4067 \\
\hline J1312-5516 & 0.849236 & -14.2434 & 6.75 & -9.67088 & -15.4015 & -33.7319 \\
\hline $\mathrm{J} 1312-6400$ & 2.43743 & -15.1656 & 2.21 & -10.1352 & -16.7817 & -34.6271 \\
\hline $\mathrm{J} 1313+0931$ & 0.848933 & -15.0969 & 0.78 & -10.5246 & -16.2549 & -33.6481 \\
\hline J1314-6101 & 2.94839 & -13.9318 & 9.6 & -8.81878 & -15.6305 & -34.1139 \\
\hline $\mathrm{J} 1316-6232$ & 0.342825 & -14.2757 & 30 & -10.0972 & -15.0399 & -34.0181 \\
\hline
\end{tabular}


Table 1 continued

\begin{tabular}{|c|c|c|c|c|c|c|}
\hline Pulsar & $P(\mathrm{~s})$ & $\log \dot{P}$ & $d(\mathrm{kpc})$ & $\log \varepsilon$ & $\log \eta$ & $\log h$ \\
\hline J1317-5759 & 2.6422 & -13.8996 & 5.55 & -8.83421 & -15.5507 & -33.7961 \\
\hline $\mathrm{J} 1317-6302$ & 0.261271 & -15.9914 & 30 & -11.9309 & -16.6376 & -35.6158 \\
\hline $\mathrm{J} 1319-6056$ & 0.284351 & -14.8153 & 12.64 & -10.718 & -15.4983 & -34.1011 \\
\hline J1319-6105 & 0.421118 & -14.8239 & 14.88 & -10.5561 & -15.6775 & -34.3511 \\
\hline $\mathrm{J} 1320-3512$ & 0.458488 & -17.7212 & 0.93 & -13.4165 & -18.6117 & -36.0813 \\
\hline J1320-5359 & 0.279729 & -14.0339 & 4.23 & -9.94367 & -14.7097 & -32.8371 \\
\hline $\mathrm{J} 1321-5922$ & 1.27906 & -14.6253 & 30 & -9.87491 & -15.9613 & -34.9395 \\
\hline $\mathrm{J} 1321+8323$ & 0.670037 & -15.2472 & 0.77 & -10.7776 & -16.3024 & -33.69 \\
\hline $\mathrm{J} 1322-6241$ & 0.506058 & -14.5867 & 19.91 & -10.239 & -15.52 & -34.3202 \\
\hline J1322-6329 & 2.76421 & -13.9547 & 30 & -8.86965 & -15.6254 & -34.6036 \\
\hline $\mathrm{J} 1324-6146$ & 0.844109 & -14.2534 & 30 & -9.68351 & -15.4089 & -34.3871 \\
\hline $\mathrm{J} 1324-6302$ & 2.4838 & -15.0092 & 11.03 & -9.97065 & -16.6335 & -35.1771 \\
\hline J1326-5859 & 0.477991 & -14.4895 & 3 & -10.1666 & -15.398 & -33.3762 \\
\hline J1326-6408 & 0.79267 & -14.5086 & 15.7 & -9.96609 & -15.6369 & -34.3338 \\
\hline J1326-6700 & 0.543009 & -14.2749 & 8.5 & -9.89665 & -15.2389 & -33.6693 \\
\hline J1327-0755 & 0.002678 & -19.752 & 2.17 & -17.6808 & -18.409 & -36.2465 \\
\hline $\mathrm{J} 1327-6222$ & 0.529913 & -13.7235 & 4 & -9.35588 & -14.6769 & -32.78 \\
\hline J1327-6301 & 0.196479 & -14.8153 & 6.5 & -10.8785 & -15.3378 & -33.6517 \\
\hline J1327-6400 & 0.280678 & -13.5058 & 30 & -9.41418 & -14.1832 & -33.1614 \\
\hline J1328-4357 & 0.532699 & -14.5214 & 2.29 & -10.1515 & -15.4771 & -33.338 \\
\hline J1328-4921 & 1.47872 & -15.2147 & 7.78 & -10.4013 & -16.6137 & -35.0057 \\
\hline J1329-6158 & 1.56522 & -13.4698 & 8.11 & -8.63177 & -14.8935 & -33.3036 \\
\hline $\mathrm{J} 1331-5245$ & 0.648117 & -15.2924 & 10.5 & -10.8373 & -16.3332 & -34.8555 \\
\hline $\mathrm{J} 1332-3032$ & 0.650434 & -15.2518 & 0.87 & -10.7952 & -16.2942 & -33.7347 \\
\hline J1333-4449 & 0.345603 & -18.2676 & 2.32 & -14.0856 & -19.0353 & -36.9019 \\
\hline J1334-5839 & 0.107718 & -16.6655 & 3.89 & -12.9898 & -16.927 & -35.018 \\
\hline J1337-6306 & 0.207953 & -15.4486 & 16.01 & -11.4871 & -15.9957 & -34.7011 \\
\hline $\mathrm{J} 1337-6423$ & 0.009423 & -19.6073 & 6.3 & -16.9897 & -18.8106 & -37.111 \\
\hline J1338-6204 & 1.23899 & -13.857 & 8.16 & -9.12046 & -15.1792 & -33.592 \\
\hline J1339-4712 & 0.137055 & -18.2757 & 1.77 & -14.4954 & -18.6418 & -36.3908 \\
\hline J1339-6618 & 0.558179 & -15.4559 & 7.62 & -11.0657 & -16.4319 & -34.8149 \\
\hline $\mathrm{J} 1340-6456$ & 0.378622 & -14.2967 & 1.92 & -10.0751 & -15.1041 & -32.8884 \\
\hline $\mathrm{J} 1341-6023$ & 0.627285 & -13.71 & 7.03 & -9.26905 & -14.7366 & -33.0846 \\
\hline $\mathrm{J} 1341-6220$ & 0.19334 & -12.5969 & 8.55 & -8.66711 & -13.1123 & -31.5454 \\
\hline $\mathrm{J} 1344-6059$ & 0.540102 & -16.3665 & 7.17 & -11.9906 & -17.3282 & -35.6847 \\
\hline J1345-6115 & 1.25309 & -14.4881 & 5.87 & -9.74668 & -15.8152 & -34.0849 \\
\hline J1346-4918 & 0.299625 & -16.4559 & 3.9 & -12.3359 & -17.1617 & -35.2538 \\
\hline J1347-5947 & 0.609962 & -13.8477 & 6.54 & -9.41896 & -14.8622 & -33.1788 \\
\hline J1348-6307 & 0.927772 & -14.4214 & 8.18 & -9.81047 & -15.618 & -34.0318 \\
\hline $\mathrm{J} 1349-6130$ & 0.259363 & -14.2899 & 5.82 & -10.2325 & -14.9329 & -33.1989 \\
\hline $\mathrm{J} 1350-5115$ & 0.295699 & -15.1232 & 4.52 & -11.0089 & -15.8232 & -33.9794 \\
\hline $\mathrm{J} 1352-6803$ & 0.628903 & -14.9101 & 14.24 & -10.4681 & -15.9378 & -34.5924 \\
\hline $\mathrm{J} 1354-6249$ & 2.95194 & -13.8297 & 5.62 & -8.71618 & -15.529 & -33.7798 \\
\hline
\end{tabular}


Table 1 continued

\begin{tabular}{|c|c|c|c|c|c|c|}
\hline Pulsar & $P(\mathrm{~s})$ & $\log \dot{P}$ & $d(\mathrm{kpc})$ & $\log \varepsilon$ & $\log \eta$ & $\log h$ \\
\hline $\mathrm{J} 1355-5153$ & 0.644301 & -14.5513 & 6.47 & -10.0988 & -15.5895 & -33.9015 \\
\hline $\mathrm{J} 1355-5747$ & 2.03867 & -15.1457 & 7.58 & -10.1929 & -16.6842 & -35.0649 \\
\hline J1355-5925 & 1.21338 & -14.2226 & 8.43 & -9.49512 & -15.5357 & -33.9626 \\
\hline $\mathrm{J} 1355-6206$ & 0.276603 & -17.5086 & 7.95 & -13.4233 & -18.1796 & -36.5811 \\
\hline J1356-5521 & 0.50738 & -15.1403 & 8.75 & -10.7915 & -16.0747 & -34.5178 \\
\hline J1357-6429 & 0.166108 & -12.4437 & 4.09 & -8.57985 & -12.8932 & -31.006 \\
\hline $\mathrm{J} 1359-6038$ & 0.127501 & -14.1979 & 5 & -10.4489 & -14.5326 & -32.7326 \\
\hline $\mathrm{J} 1400-6325$ & 0.031182 & -13.4101 & 7 & -10.2727 & -13.1331 & -31.4793 \\
\hline $\mathrm{J} 1401-6357$ & 0.84279 & -13.7773 & 1.8 & -9.20811 & -14.9322 & -32.6885 \\
\hline $\mathrm{J} 1403-6310$ & 0.39917 & -16.0458 & 6.14 & -11.8011 & -16.8761 & -35.1653 \\
\hline $\mathrm{J} 1403-7646$ & 1.3062 & -14.9208 & 7.03 & -10.1614 & -16.266 & -34.614 \\
\hline $\mathrm{J} 1405-4656$ & 0.007602 & -19.5544 & 0.74 & -17.03 & -18.6645 & -36.0348 \\
\hline $\mathrm{J} 1405-5641$ & 0.617575 & -14.9208 & 21.3 & -10.4867 & -15.9407 & -34.7701 \\
\hline J1406-5806 & 0.288349 & -15.214 & 6.73 & -11.1106 & -15.903 & -34.2321 \\
\hline J1406-6121 & 0.213075 & -13.262 & 9.11 & -9.29003 & -13.8197 & -32.2803 \\
\hline J1407-6048 & 0.492344 & -14.5003 & 9.7 & -10.1646 & -15.4217 & -33.9096 \\
\hline $\mathrm{J} 1407-6153$ & 0.701615 & -14.0531 & 9.75 & -9.56351 & -15.1283 & -33.6184 \\
\hline J1409-6953 & 0.528591 & -15.0757 & 12.6 & -10.7091 & -16.028 & -34.6294 \\
\hline $\mathrm{J} 1410-6132$ & 0.050052 & -13.4949 & 30 & -10.152 & -13.4234 & -32.4016 \\
\hline J1410-7404 & 0.278729 & -17.1713 & 2.15 & -13.0827 & -17.8457 & -35.6792 \\
\hline $\mathrm{J} 1412-6111$ & 0.529156 & -14.719 & 5.95 & -10.3519 & -15.6717 & -33.9473 \\
\hline $\mathrm{J} 1412-6145$ & 0.315225 & -13.0057 & 9.32 & -8.86361 & -13.7335 & -32.2039 \\
\hline $\mathrm{J} 1413-6141$ & 0.285625 & -12.4776 & 11 & -8.37831 & -13.1625 & -31.705 \\
\hline $\mathrm{J} 1413-6222$ & 0.292408 & -14.6517 & 27.74 & -10.5423 & -15.3468 & -34.291 \\
\hline J1413-6307 & 0.394946 & -14.129 & 3.68 & -9.88902 & -14.9547 & -33.0216 \\
\hline $\mathrm{J} 1414-6802$ & 4.63019 & -14.1945 & 6.6 & -8.88545 & -16.0892 & -34.4098 \\
\hline $\mathrm{J} 1415-6621$ & 0.392479 & -15.2366 & 14.4 & -10.9993 & -16.0595 & -34.719 \\
\hline J1416-5033 & 0.794883 & -15.9208 & 1.99 & -11.3771 & -17.0503 & -34.8502 \\
\hline J1416-6037 & 0.29558 & -14.3686 & 5.72 & -10.2544 & -15.0684 & -33.3268 \\
\hline J1418-3921 & 1.09681 & -15.0511 & 5.04 & -10.3675 & -16.3204 & -34.5239 \\
\hline J1418-5945 & 1.6726 & -15.567 & 9.31 & -10.7002 & -17.0196 & -35.4896 \\
\hline J1420-5416 & 0.935772 & -15.6253 & 4.9 & -11.0106 & -16.8256 & -35.0168 \\
\hline $\mathrm{J} 1420-5625$ & 0.034117 & -19.1707 & 1.74 & -15.9943 & -18.9328 & -36.6744 \\
\hline $\mathrm{J} 1420-6048$ & 0.06818 & -13.0799 & 7.65 & -9.60277 & -13.1427 & -31.5274 \\
\hline $\mathrm{J} 1423-6953$ & 0.333411 & -14.8386 & 6.38 & -10.6722 & -15.5908 & -33.8966 \\
\hline J1424-5556 & 0.770375 & -15.1079 & 7.71 & -10.5778 & -16.2238 & -34.6119 \\
\hline J1424-5822 & 0.366734 & -14.4045 & 10.19 & -10.1967 & -15.198 & -33.7072 \\
\hline J1424-6438 & 1.0235 & -15.6198 & 8.31 & -10.9662 & -16.859 & -35.2797 \\
\hline $\mathrm{J} 1425-5723$ & 0.353263 & -16.6576 & 1.46 & -12.466 & -17.4348 & -35.1002 \\
\hline $\mathrm{J} 1425-5759$ & 0.707868 & -15.1296 & 10.81 & -10.6362 & -16.2087 & -34.7436 \\
\hline $\mathrm{J} 1425-6210$ & 0.50173 & -15.3206 & 9.98 & -10.9766 & -16.2502 & -34.7504 \\
\hline J1427-4158 & 0.586486 & -15.2069 & 5.89 & -10.7952 & -16.2043 & -34.4755 \\
\hline $\mathrm{J} 1428-5530$ & 0.57029 & -14.6819 & 2.29 & -10.2824 & -15.6672 & -33.5281 \\
\hline J1429-5935 & 0.763915 & -13.3686 & 10.62 & -8.84206 & -14.4807 & -33.0079 \\
\hline $\mathrm{J} 1430-6623$ & 0.785441 & -14.5575 & 1.8 & -10.019 & -15.6818 & -33.4381 \\
\hline
\end{tabular}


Table 1 continued

\begin{tabular}{|c|c|c|c|c|c|c|}
\hline Pulsar & $P(\mathrm{~s})$ & $\log \dot{P}$ & $d(\mathrm{kpc})$ & $\log \varepsilon$ & $\log \eta$ & $\log h$ \\
\hline $\mathrm{J} 1431-4715$ & 0.002012 & -19.8508 & 2.42 & -17.9037 & -18.3836 & -36.2684 \\
\hline $\mathrm{J} 1431-5740$ & 0.004111 & -20.1925 & 4.07 & -17.9351 & -19.0356 & -37.1462 \\
\hline $\mathrm{J} 1432-5032$ & 2.03499 & -14.2277 & 5.72 & -9.27566 & -15.7654 & -34.0238 \\
\hline $\mathrm{J} 1433-6038$ & 1.95443 & -14.2495 & 9.74 & -9.31502 & -15.7697 & -34.2593 \\
\hline $\mathrm{J} 1434-6006$ & 0.306368 & -14.52 & 7.25 & -10.3903 & -15.2354 & -33.5968 \\
\hline $\mathrm{J} 1434-6029$ & 0.963348 & -14.9872 & 5.78 & -10.3599 & -16.2001 & -34.4631 \\
\hline $\mathrm{J} 1434+7257$ & 0.041741 & -18.2596 & 0.75 & -14.9956 & -18.1093 & -35.4855 \\
\hline J1435-5954 & 0.472995 & -14.8125 & 1.46 & -10.4942 & -15.7165 & -33.3819 \\
\hline $\mathrm{J} 1435-6100$ & 0.009348 & -19.6108 & 3.25 & -16.9967 & -18.8107 & -36.8236 \\
\hline J1437-5959 & 0.061696 & -14.066 & 11.12 & -10.6323 & -14.0854 & -32.6326 \\
\hline $\mathrm{J} 1437-6146$ & 0.467616 & -14.1986 & 4.77 & -9.88525 & -15.0976 & -33.2772 \\
\hline $\mathrm{J} 1439-5501$ & 0.028635 & -18.8477 & 0.76 & -15.7474 & -18.5338 & -35.9156 \\
\hline J1440-6344 & 0.459605 & -14.9508 & 4.03 & -10.6449 & -15.8423 & -33.9487 \\
\hline J1441-6137 & 1.17584 & -15.4437 & 4.44 & -10.7299 & -16.7432 & -34.8916 \\
\hline $\mathrm{J} 1443-5122$ & 0.732061 & -15.4711 & 3.17 & -10.9631 & -16.5648 & -34.5669 \\
\hline J1444-5941 & 2.76023 & -14.0846 & 4.38 & -9.0002 & -15.7547 & -33.8972 \\
\hline J1444-6026 & 4.75858 & -13.7328 & 8.78 & -8.4119 & -15.6395 & -34.084 \\
\hline J1446-4701 & 0.002195 & -20.0083 & 2.03 & -18.0234 & -18.5789 & -36.3875 \\
\hline J1449-5846 & 0.46333 & -16.0655 & 4.74 & -11.7562 & -16.9605 & -35.1374 \\
\hline $\mathrm{J} 1452-5851$ & 0.386625 & -13.295 & 5.64 & -9.06425 & -14.1114 & -32.3638 \\
\hline J1452-6036 & 0.154991 & -14.8386 & 9.44 & -11.0049 & -15.2581 & -33.7341 \\
\hline $\mathrm{J} 1453+1902$ & 0.005792 & -19.9355 & 0.95 & -17.5293 & -18.9275 & -36.4063 \\
\hline $\mathrm{J} 1453-6413$ & 0.179485 & -14.5607 & 2.8 & -10.6632 & -15.0438 & -32.9921 \\
\hline J1454-5846 & 0.045249 & -18.0878 & 3.32 & -14.7887 & -17.9725 & -35.9947 \\
\hline $\mathrm{J} 1455-3330$ & 0.007987 & -19.6144 & 0.74 & -17.0686 & -18.7459 & -36.1162 \\
\hline $\mathrm{J} 1456-6843$ & 0.263377 & -16.0074 & 0.43 & -11.9434 & -16.6572 & -33.7917 \\
\hline $\mathrm{J} 1457-5122$ & 1.7483 & -14.2765 & 1.37 & -9.39048 & -15.7483 & -33.3861 \\
\hline J1457-5900 & 1.49864 & -14.4365 & 4.25 & -9.61737 & -15.8414 & -33.9708 \\
\hline J1457-5902 & 0.390739 & -13.9101 & 11.33 & -9.67476 & -14.7311 & -33.2864 \\
\hline $\mathrm{J} 1501-0046$ & 0.464037 & -15.6216 & 2.37 & -11.3116 & -16.5173 & -34.3931 \\
\hline J1501-5637 & 0.782949 & -15.7696 & 6.75 & -11.2324 & -16.8924 & -35.2228 \\
\hline $\mathrm{J} 1502-5653$ & 0.535504 & -14.7375 & 4.51 & -10.3653 & -15.6955 & -33.8507 \\
\hline $\mathrm{J} 1502-5828$ & 0.668105 & -13.4389 & 12.21 & -8.9706 & -14.4929 & -33.0807 \\
\hline $\mathrm{J} 1502-6128$ & 0.842104 & -14.8697 & 7.88 & -10.3008 & -16.0242 & -34.4218 \\
\hline $\mathrm{J} 1502-6752$ & 0.026744 & -18.5017 & 11.57 & -15.431 & -18.1581 & -36.7225 \\
\hline $\mathrm{J} 1503+2111$ & 3.314 & -15.8539 & 0.72 & -10.6901 & -17.6034 & -34.9618 \\
\hline $\mathrm{J} 1504-5621$ & 0.412985 & -14.2573 & 3.93 & -9.99789 & -15.1024 & -33.1978 \\
\hline J1506-5158 & 0.840739 & -14.1959 & 1.67 & -9.62775 & -15.3497 & -33.0734 \\
\hline $\mathrm{J} 1507-4352$ & 0.286757 & -14.7959 & 1.79 & -10.6949 & -15.4825 & -33.2365 \\
\hline J1507-6640 & 0.355655 & -14.9355 & 5.62 & -10.7411 & -15.7157 & -33.9665 \\
\hline $\mathrm{J} 1509+5531$ & 0.739682 & -14.301 & 2.1 & -9.78853 & -15.3992 & -33.2225 \\
\hline $\mathrm{J} 1509-5850$ & 0.088922 & -14.0376 & 3.85 & -10.4452 & -14.2158 & -32.3023 \\
\hline $\mathrm{J} 1509-6015$ & 0.339038 & -14.6737 & 13.63 & -10.5 & -15.4331 & -34.0686 \\
\hline J1510-4422 & 0.943871 & -15.2147 & 4.22 & -10.5963 & -16.4187 & -34.5451 \\
\hline $\mathrm{J} 1511-5414$ & 0.200384 & -15.3143 & 2.24 & -11.3689 & -15.8453 & -33.6966 \\
\hline $\mathrm{J} 1511-5835$ & 0.301511 & -15.4634 & 7.1 & -11.3407 & -16.1719 & -34.5242 \\
\hline
\end{tabular}


Table 1 continued

\begin{tabular}{|c|c|c|c|c|c|c|}
\hline Pulsar & $P(\mathrm{~s})$ & $\log \dot{P}$ & $d(\mathrm{kpc})$ & $\log \varepsilon$ & $\log \eta$ & $\log h$ \\
\hline $\mathrm{J} 1512-5431$ & 2.04053 & -14.7167 & 6.61 & -9.7635 & -16.2556 & -34.5769 \\
\hline J1512-5759 & 0.128694 & -14.1643 & 12.7 & -10.4113 & -14.503 & -33.1079 \\
\hline J1513-5739 & 0.973458 & -13.5607 & 9.84 & -8.9289 & -14.7781 & -33.2722 \\
\hline J1513-5908 & 0.151251 & -11.8153 & 4.4 & -7.99216 & -12.2242 & -30.3687 \\
\hline J1513-5946 & 1.04612 & -14.0691 & 4.26 & -9.40602 & -15.3178 & -33.4482 \\
\hline J1514-4834 & 0.454839 & -15.0339 & 1.59 & -10.7325 & -15.9209 & -33.6233 \\
\hline J1514-4946 & 0.003589 & -19.7305 & 1.27 & -17.5321 & -18.5146 & -36.1195 \\
\hline J1514-5925 & 0.148796 & -14.5406 & 4.5 & -10.7246 & -14.9423 & -33.0966 \\
\hline $\mathrm{J} 1515-5720$ & 0.286646 & -14.2147 & 10.32 & -10.1139 & -14.9012 & -33.4159 \\
\hline $\mathrm{J} 1517-4356$ & 0.650837 & -15.6655 & 4.44 & -11.2086 & -16.7082 & -34.8566 \\
\hline $\mathrm{J} 1517-4636$ & 0.886612 & -14.6778 & 10.98 & -10.0866 & -15.8547 & -34.3963 \\
\hline $\mathrm{J} 1518-0627$ & 0.794997 & -15.3788 & 2.69 & -10.835 & -16.5083 & -34.4391 \\
\hline $\mathrm{J} 1518+4904$ & 0.040935 & -19.5654 & 0.7 & -16.3099 & -19.4067 & -36.7528 \\
\hline J1518-5415 & 0.214925 & -16.3915 & 4.26 & -12.4157 & -16.9529 & -35.0834 \\
\hline $\mathrm{J} 1519-5734$ & 0.518758 & -14.3696 & 13.12 & -10.0112 & -15.3137 & -33.9327 \\
\hline J1519-6106 & 2.15431 & -14.0773 & 7.23 & -9.10051 & -15.6397 & -33.9999 \\
\hline $\mathrm{J} 1519-6308$ & 1.25405 & -14.2248 & 17.81 & -9.48299 & -15.5522 & -34.3039 \\
\hline $\mathrm{J} 1522-5525$ & 1.3896 & -14.4815 & 1.89 & -9.69514 & -15.8535 & -33.631 \\
\hline J1522-5829 & 0.395353 & -14.699 & 4.47 & -10.4585 & -15.5251 & -33.6765 \\
\hline $\mathrm{J} 1524-5625$ & 0.078219 & -13.4089 & 3.84 & -9.87217 & -13.5314 & -31.6168 \\
\hline J1524-5706 & 1.11605 & -12.4486 & 21.59 & -7.75741 & -13.7254 & -32.5607 \\
\hline J1524-5819 & 0.961043 & -12.8996 & 10.87 & -8.27343 & -14.1115 & -32.6488 \\
\hline $\mathrm{J} 1525-5417$ & 1.01169 & -13.7905 & 6.01 & -9.14198 & -15.0247 & -33.3046 \\
\hline $\mathrm{J} 1525-5523$ & 0.355156 & -17.0477 & 3.5 & -12.8538 & -17.8273 & -35.8724 \\
\hline $\mathrm{J} 1525-5545$ & 0.01136 & -18.8827 & 3.53 & -16.1839 & -18.1673 & -36.2161 \\
\hline $\mathrm{J} 1525-5605$ & 0.280349 & -15.9355 & 6.77 & -11.8444 & -16.6124 & -34.944 \\
\hline J1526-5633 & 0.301888 & -15.9101 & 6.44 & -11.7868 & -16.6191 & -34.929 \\
\hline J1527-3931 & 2.41758 & -13.719 & 2.01 & -8.69213 & -15.3315 & -33.1358 \\
\hline $\mathrm{J} 1527-5552$ & 1.0487 & -13.9469 & 7.15 & -9.28282 & -15.1967 & -33.5521 \\
\hline J1528-3146 & 0.060822 & -18.6038 & 0.99 & -15.1763 & -18.617 & -36.1137 \\
\hline J1528-4109 & 0.526556 & -15.4023 & 5.96 & -11.0374 & -16.3529 & -34.6292 \\
\hline J1528-5547 & 3.4673 & -14.1107 & 5.55 & -8.92725 & -15.8798 & -34.1252 \\
\hline $\mathrm{J} 1529-3828$ & 0.008486 & -19.5686 & 4.85 & -16.9965 & -18.7265 & -36.9133 \\
\hline $\mathrm{J} 1529-5355$ & 0.891265 & -15.0915 & 7.75 & -10.4981 & -16.2707 & -34.661 \\
\hline J1529-5611 & 0.822249 & -14.3872 & 3.77 & -9.82876 & -15.5314 & -33.6088 \\
\hline $\mathrm{J} 1530-5327$ & 0.278957 & -14.3298 & 1.46 & -10.2408 & -15.0044 & -32.6699 \\
\hline $\mathrm{J} 1531-4012$ & 0.356849 & -16.0164 & 7.75 & -11.8204 & -16.798 & -35.1884 \\
\hline $\mathrm{J} 1531-5610$ & 0.084202 & -13.8633 & 3.1 & -10.2945 & -14.0177 & -32.0102 \\
\hline $\mathrm{J} 1532+2745$ & 1.12484 & -15.1079 & 0.98 & -10.4134 & -16.3881 & -33.8804 \\
\hline $\mathrm{J} 1532-5308$ & 0.443825 & -16.1871 & 4.45 & -11.8964 & -17.0634 & -35.2129 \\
\hline J1534-4428 & 1.22143 & -15.7447 & 10.94 & -11.0144 & -17.0607 & -35.6008 \\
\hline J1534-5334 & 1.36888 & -14.8447 & 1.13 & -10.0648 & -16.2102 & -33.7643 \\
\hline J1534-5405 & 0.289689 & -14.8125 & 4.26 & -10.7071 & -15.5036 & -33.634 \\
\hline J1535-4114 & 0.432866 & -14.3904 & 2.8 & -10.1106 & -15.2559 & -33.2041 \\
\hline J1535-4415 & 0.468402 & -16.3925 & 5.38 & -12.0785 & -17.2923 & -35.5242 \\
\hline $\mathrm{J} 1535-5450$ & 0.566734 & -13.8447 & 4.63 & -9.44783 & -14.8272 & -32.9938 \\
\hline
\end{tabular}


Table 1 continued

\begin{tabular}{|c|c|c|c|c|c|c|}
\hline Pulsar & $P(\mathrm{~s})$ & $\log \dot{P}$ & $d(\mathrm{kpc})$ & $\log \varepsilon$ & $\log \eta$ & $\log h$ \\
\hline $\mathrm{J} 1535-5848$ & 0.307178 & -14.5654 & 3.13 & -10.4346 & -15.282 & -33.2786 \\
\hline J1536-3602 & 1.31976 & -15.1024 & 6.45 & -10.3384 & -16.452 & -34.7626 \\
\hline J1536-5433 & 0.881438 & -14.719 & 3.72 & -10.1303 & -15.8933 & -33.9649 \\
\hline J1536-5907 & 0.557841 & -14.8633 & 12.09 & -10.4733 & -15.8389 & -34.4224 \\
\hline $\mathrm{J} 1537+1155$ & 0.037904 & -17.6162 & 1.05 & -14.394 & -17.424 & -34.9463 \\
\hline $\mathrm{J} 1537-4912$ & 0.301311 & -14.7144 & 1.82 & -10.592 & -15.4226 & -33.1837 \\
\hline $\mathrm{J} 1537-5153$ & 1.52812 & -14.3799 & 2.63 & -9.55225 & -15.7932 & -33.7142 \\
\hline J1537-5645 & 0.430464 & -14.556 & 24.59 & -10.2786 & -15.419 & -34.3109 \\
\hline $\mathrm{J} 1538+2345$ & 3.44938 & -14.1618 & 0.98 & -8.98059 & -15.9287 & -33.421 \\
\hline $\mathrm{J} 1538-5438$ & 0.276726 & -14.8477 & 3.6 & -10.7622 & -15.5189 & -33.5763 \\
\hline J1538-5519 & 0.395731 & -16.3872 & 9.7 & -12.1464 & -17.2138 & -35.7016 \\
\hline $\mathrm{J} 1538-5551$ & 0.104675 & -14.4935 & 10.4 & -10.8302 & -14.7425 & -33.2606 \\
\hline $\mathrm{J} 1538-5621$ & 1.90849 & -14.1361 & 3.76 & -9.21194 & -15.6459 & -33.7222 \\
\hline $\mathrm{J} 1538-5638$ & 0.84398 & -14.1475 & 12.94 & -9.57774 & -15.303 & -33.916 \\
\hline $\mathrm{J} 1538-5732$ & 0.341213 & -14.342 & 3.88 & -10.1655 & -15.1042 & -33.1941 \\
\hline $\mathrm{J} 1538-5750$ & 0.506568 & -16.3768 & 2.41 & -12.0287 & -17.3105 & -35.1936 \\
\hline J1539-4828 & 1.27284 & -14.8962 & 3.74 & -10.148 & -16.2301 & -34.304 \\
\hline J1539-5521 & 1.00496 & -15.1379 & 6.84 & -10.4923 & -16.3692 & -34.7053 \\
\hline J1539-5626 & 0.243392 & -14.3143 & 4 & -10.2845 & -14.9297 & -33.0328 \\
\hline J1539-6322 & 1.63085 & -15.6946 & 7.75 & -10.8388 & -17.1362 & -35.5266 \\
\hline J1540-5736 & 0.612916 & -15.3757 & 8.17 & -10.9449 & -16.3923 & -34.8055 \\
\hline $\mathrm{J} 1541-5535$ & 0.295838 & -13.1249 & 7.46 & -9.01043 & -13.8251 & -32.1989 \\
\hline $\mathrm{J} 1542-5034$ & 0.599245 & -14.4012 & 2.58 & -9.98015 & -15.408 & -33.3206 \\
\hline J1542-5133 & 1.78387 & -15.2299 & 4.87 & -10.3351 & -16.7104 & -34.899 \\
\hline $\mathrm{J} 1542-5303$ & 1.20757 & -13.109 & 6.02 & -8.38366 & -14.4201 & -32.7007 \\
\hline $\mathrm{J} 1543-0620$ & 0.709064 & -15.0555 & 1.27 & -10.5614 & -16.1354 & -33.7402 \\
\hline $\mathrm{J} 1543+0929$ & 0.748448 & -15.3645 & 5.9 & -10.8469 & -16.4678 & -34.7397 \\
\hline J1543-5013 & 0.644255 & -13.9957 & 6.76 & -9.54317 & -15.0339 & -33.3649 \\
\hline J1543-5149 & 0.002057 & -19.7905 & 1.46 & -17.8338 & -18.3329 & -35.9983 \\
\hline J1543-5459 & 0.377119 & -13.284 & 6.32 & -9.06407 & -14.0896 & -32.3914 \\
\hline $\mathrm{J} 1544+4937$ & 0.002159 & -20.5331 & 2.3 & -18.5554 & -19.0965 & -36.9593 \\
\hline $\mathrm{J} 1544-5308$ & 0.178554 & -16.2168 & 1.29 & -12.3216 & -16.6977 & -34.3094 \\
\hline $\mathrm{J} 1545-4550$ & 0.003575 & -19.2798 & 2.01 & -17.0831 & -18.0623 & -35.8665 \\
\hline $\mathrm{J} 1546-5302$ & 0.58084 & -13.9281 & 6.1 & -9.52061 & -14.9213 & -33.2077 \\
\hline J1547-0944 & 1.57692 & -14.5317 & 3.18 & -9.69039 & -15.9586 & -33.9621 \\
\hline $\mathrm{J} 1547-5750$ & 0.647198 & -16.5918 & 3.91 & -12.1373 & -17.6319 & -35.7252 \\
\hline J1547-5839 & 0.24219 & -15.2262 & 7.19 & -11.1986 & -15.8395 & -34.1973 \\
\hline J1548-4821 & 0.145655 & -18.0969 & 3.76 & -14.2901 & -18.4894 & -36.5656 \\
\hline J1548-4927 & 0.602738 & -14.3925 & 3.89 & -9.96896 & -15.4018 & -33.4928 \\
\hline $\mathrm{J} 1548-5607$ & 0.170934 & -13.9706 & 6.95 & -10.0943 & -14.4326 & -32.7756 \\
\hline $\mathrm{J} 1549+2113$ & 1.26247 & -15.0685 & 2.33 & -10.3239 & -16.3989 & -34.2673 \\
\hline J1549-4848 & 0.288347 & -13.8508 & 1.54 & -9.74741 & -14.5398 & -32.2284 \\
\hline
\end{tabular}


Table 1 continued

\begin{tabular}{|c|c|c|c|c|c|c|}
\hline Pulsar & $P(\mathrm{~s})$ & $\log \dot{P}$ & $d(\mathrm{kpc})$ & $\log \varepsilon$ & $\log \eta$ & $\log h$ \\
\hline J1549-5722 & 0.497772 & -16.3279 & 2.93 & -11.9874 & -17.2541 & -35.222 \\
\hline $\mathrm{J} 1550-5242$ & 0.749659 & -13.7496 & 6.67 & -9.23126 & -14.8536 & -33.1788 \\
\hline $\mathrm{J} 1550-5317$ & 1.42112 & -15.0292 & 8.45 & -10.2331 & -16.411 & -34.8389 \\
\hline $\mathrm{J} 1550-5418$ & 2.06983 & -10.6345 & 4 & -5.67512 & -12.1796 & -30.2827 \\
\hline $\mathrm{J} 1551-4424$ & 0.67406 & -15.7258 & 1.98 & -11.2537 & -16.7837 & -34.5814 \\
\hline $\mathrm{J} 1551-5310$ & 0.453394 & -12.71 & 7.52 & -8.41004 & -13.5956 & -31.9729 \\
\hline J1552-4937 & 0.006284 & -19.7212 & 3.36 & -17.2796 & -18.7486 & -36.776 \\
\hline $\mathrm{J} 1553-5456$ & 1.08133 & -13.8041 & 4.8 & -9.12669 & -15.0672 & -33.2495 \\
\hline J1554-5209 & 0.12523 & -14.6402 & 3.44 & -10.899 & -14.967 & -33.0046 \\
\hline $\mathrm{J} 1554-5512$ & 3.41804 & -13.5058 & 8.05 & -8.32862 & -15.2688 & -33.6756 \\
\hline $\mathrm{J} 1555-0515$ & 0.97541 & -14.6882 & 1.66 & -10.0556 & -15.9066 & -33.6277 \\
\hline $\mathrm{J} 1555-2341$ & 0.532578 & -15.1586 & 4.61 & -10.7888 & -16.1142 & -34.2789 \\
\hline $\mathrm{J} 1555-3134$ & 0.51811 & -16.2062 & 6.1 & -11.8483 & -17.1498 & -35.4362 \\
\hline J1556-5358 & 0.994681 & -13.983 & 6.96 & -9.34183 & -15.2098 & -33.5535 \\
\hline $\mathrm{J} 1557-4258$ & 0.329187 & -15.4815 & 7.53 & -11.3206 & -16.2281 & -34.6059 \\
\hline J1558-5419 & 0.594575 & -14.219 & 9.09 & -9.8013 & -15.2223 & -33.6819 \\
\hline $\mathrm{J} 1558-5756$ & 1.12234 & -12.7305 & 3.74 & -8.03691 & -14.0098 & -32.0837 \\
\hline $\mathrm{J} 1559-4438$ & 0.257056 & -14.9914 & 2.3 & -10.9379 & -15.6306 & -33.4934 \\
\hline $\mathrm{J} 1559-5545$ & 0.957242 & -13.6882 & 5.09 & -9.06377 & -14.8984 & -33.1062 \\
\hline $\mathrm{J} 1600-3053$ & 0.003598 & -20.0223 & 1.8 & -17.8228 & -18.8075 & -36.5638 \\
\hline $\mathrm{J} 1600-5044$ & 0.192601 & -14.2958 & 6.9 & -10.3677 & -14.8097 & -33.1496 \\
\hline $\mathrm{J} 1600-5751$ & 0.194454 & -14.6716 & 5.32 & -10.7394 & -15.1896 & -33.4166 \\
\hline J1600-5916 & 1.24767 & -15.0521 & 6.54 & -10.3125 & -16.3773 & -34.694 \\
\hline $\mathrm{J} 1601-5244$ & 2.55936 & -15.1421 & 5.14 & -10.0905 & -16.7793 & -34.9914 \\
\hline $\mathrm{J} 1601-5335$ & 0.288457 & -13.2048 & 4.04 & -9.10128 & -13.894 & -32.0015 \\
\hline $\mathrm{J} 1602-4957$ & 0.81999 & -13.7986 & 6.83 & -9.24134 & -14.9416 & -33.277 \\
\hline $\mathrm{J} 1602-5100$ & 0.864227 & -13.1574 & 8 & -8.57731 & -14.3232 & -32.7273 \\
\hline $\mathrm{J} 1603-2531$ & 0.28307 & -14.7986 & 4.6 & -10.7033 & -15.4796 & -33.6435 \\
\hline $\mathrm{J} 1603-2712$ & 0.778313 & -14.5214 & 2.58 & -9.98683 & -15.6417 & -33.5544 \\
\hline J1603-3539 & 0.141909 & -15.9066 & 3.83 & -12.1111 & -16.2877 & -34.372 \\
\hline $\mathrm{J} 1603-5657$ & 0.496077 & -14.5544 & 8.52 & -10.2154 & -15.4791 & -33.9106 \\
\hline $\mathrm{J} 1603-7202$ & 0.014842 & -19.8069 & 0.53 & -16.9919 & -19.2075 & -36.4329 \\
\hline $\mathrm{J} 1604-4718$ & 0.527466 & -14.3045 & 1.48 & -9.93887 & -15.2559 & -32.9272 \\
\hline J1604-4909 & 0.327418 & -14.9914 & 3.59 & -10.8328 & -15.7357 & -33.7918 \\
\hline $\mathrm{J} 1604-7203$ & 0.341403 & -16.0757 & 2.56 & -11.899 & -16.8381 & -34.7474 \\
\hline $\mathrm{J} 1605-5215$ & 1.01361 & -14.3233 & 7.06 & -9.67398 & -15.5583 & -33.9082 \\
\hline $\mathrm{J} 1605-5257$ & 0.658013 & -15.5918 & 1.28 & -11.1301 & -16.6391 & -34.2474 \\
\hline $\mathrm{J} 1607-0032$ & 0.421816 & -15.5143 & 0.61 & -11.2457 & -16.3685 & -33.6549 \\
\hline $\mathrm{J} 1607-5140$ & 0.342723 & -14.5952 & 7.01 & -10.4168 & -15.3593 & -33.706 \\
\hline J1607-6449 & 0.298116 & -16.6038 & 3.82 & -12.486 & -17.3073 & -35.3905 \\
\hline J1609-1930 & 1.55792 & -15.2933 & 2.33 & -10.4573 & -16.715 & -34.5834 \\
\hline $\mathrm{J} 1609-4616$ & 0.249609 & -15.3002 & 4.12 & -11.2594 & -15.9266 & -34.0425 \\
\hline $\mathrm{J} 1609-5158$ & 1.2794 & -13.8861 & 12.73 & -9.1356 & -15.2222 & -33.8281 \\
\hline
\end{tabular}


Table 1 continued

\begin{tabular}{|c|c|c|c|c|c|c|}
\hline Pulsar & $P(\mathrm{~s})$ & $\log \dot{P}$ & $d(\mathrm{kpc})$ & $\log \varepsilon$ & $\log \eta$ & $\log h$ \\
\hline $\mathrm{J} 1610-1322$ & 1.01839 & -15.6383 & 3.89 & -10.9869 & -16.8753 & -34.9663 \\
\hline $\mathrm{J} 1610-5006$ & 0.481119 & -13.8665 & 6.59 & -9.54076 & -14.7779 & -33.0978 \\
\hline $\mathrm{J} 1610-5303$ & 0.786468 & -14.5817 & 6.6 & -10.0426 & -15.7065 & -34.0271 \\
\hline $\mathrm{J} 1611-4811$ & 1.29685 & -14.71 & 5.41 & -9.95362 & -16.052 & -34.2863 \\
\hline J1611-4949 & 0.666438 & -15.266 & 8.82 & -10.7988 & -16.3189 & -34.7654 \\
\hline J1611-5209 & 0.182492 & -14.2865 & 3.33 & -10.3818 & -14.7769 & -32.8004 \\
\hline $\mathrm{J} 1611-5847$ & 0.35455 & -17.6968 & 2.33 & -13.5037 & -18.4756 & -36.344 \\
\hline $\mathrm{J} 1612+2008$ & 0.426646 & -16.4283 & 1.43 & -12.1548 & -17.2875 & -34.9439 \\
\hline $\mathrm{J} 1612-2408$ & 0.923834 & -14.8041 & 3.12 & -10.1951 & -15.9988 & -33.9941 \\
\hline J1612-5136 & 0.483311 & -14.4225 & 18.18 & -10.0948 & -15.3359 & -34.0965 \\
\hline $\mathrm{J} 1612-5805$ & 0.61552 & -15.0292 & 6.29 & -10.5965 & -16.0476 & -34.3473 \\
\hline $\mathrm{J} 1613-4714$ & 0.382376 & -15.1993 & 3.89 & -10.9733 & -16.0109 & -34.1019 \\
\hline $\mathrm{J} 1613-5211$ & 0.457502 & -13.7167 & 6.14 & -9.41285 & -14.6062 & -32.8955 \\
\hline $\mathrm{J} 1613-5234$ & 0.655221 & -14.1785 & 9.94 & -9.71865 & -15.224 & -33.7225 \\
\hline $\mathrm{J} 1614+0737$ & 1.2068 & -14.6271 & 1.77 & -9.902 & -15.9379 & -33.6869 \\
\hline $\mathrm{J} 1614-2230$ & 0.003151 & -20.0168 & 0.7 & -17.8749 & -18.7444 & -36.0906 \\
\hline J1614-3937 & 0.407292 & -15.7986 & 10.1 & -11.5452 & -16.6377 & -35.143 \\
\hline J1614-5048 & 0.231694 & -12.3054 & 7.24 & -8.29703 & -12.8995 & -31.2603 \\
\hline J1614-5144 & 1.53401 & -14.1284 & 9.56 & -9.29915 & -15.5434 & -34.0249 \\
\hline J1614-5402 & 0.572592 & -16.5376 & 6.96 & -12.1363 & -17.5246 & -35.8683 \\
\hline J1615-2940 & 2.47757 & -14.8013 & 1.88 & -9.76386 & -16.4245 & -34.1997 \\
\hline J1615-5444 & 0.360958 & -15.4711 & 8.4 & -11.2702 & -16.2577 & -34.683 \\
\hline J1615-5537 & 0.791526 & -14.699 & 3.57 & -10.1571 & -15.8266 & -33.8803 \\
\hline J1616-5017 & 0.491384 & -13.3316 & 3.92 & -8.99674 & -14.2522 & -32.3465 \\
\hline J1616-5109 & 1.21959 & -13.719 & 18.97 & -8.9893 & -15.0343 & -33.8135 \\
\hline J1616-5208 & 1.02583 & -13.5391 & 7.38 & -8.88457 & -14.7793 & -33.1484 \\
\hline $\mathrm{J} 1617-4216$ & 3.42847 & -13.7423 & 6.28 & -8.56377 & -15.5066 & -33.8056 \\
\hline J1617-4608 & 0.56708 & -13.7825 & 10.59 & -9.38542 & -14.7653 & -33.2913 \\
\hline $\mathrm{J} 1617-5055$ & 0.069357 & -12.8697 & 6.46 & -9.38512 & -12.9399 & -31.2512 \\
\hline J1618-4723 & 0.203553 & -14.7011 & 3.42 & -10.749 & -15.239 & -33.2741 \\
\hline $\mathrm{J} 1620-5414$ & 1.15636 & -16.1713 & 1.73 & -11.4648 & -17.4636 & -35.2027 \\
\hline J1621-5039 & 1.08402 & -13.8861 & 4.88 & -9.20757 & -15.1502 & -33.3397 \\
\hline $\mathrm{J} 1621-5243$ & 0.371924 & -15.1146 & 7.76 & -10.9007 & -15.9142 & -34.3052 \\
\hline $\mathrm{J} 1622-3751$ & 0.731463 & -14.5901 & 11.55 & -10.0824 & -15.6834 & -34.247 \\
\hline $\mathrm{J} 1622-4332$ & 0.916938 & -14.8069 & 8.32 & -10.2011 & -15.9984 & -34.4195 \\
\hline $\mathrm{J} 1622-4347$ & 0.457681 & -14.2967 & 5.37 & -9.99269 & -15.1864 & -33.4175 \\
\hline $\mathrm{J} 1622-4802$ & 0.265072 & -15.5129 & 6 & -11.446 & -16.1654 & -34.4446 \\
\hline $\mathrm{J} 1622-4845$ & 0.735092 & -15.9586 & 5.24 & -11.4488 & -17.0541 & -35.2745 \\
\hline J1622-4944 & 1.07297 & -13.767 & 8.55 & -9.09296 & -15.0267 & -33.4598 \\
\hline $\mathrm{J} 1622-4950$ & 4.3261 & -10.7696 & 9.14 & -5.49 & -12.6348 & -31.0968 \\
\hline J1622-6617 & 0.023623 & -19.2306 & 4.66 & -16.2138 & -18.8331 & -37.0026 \\
\hline J1623-0841 & 0.503015 & -14.7077 & 3.83 & -10.3627 & -15.6385 & -33.7227 \\
\hline J1623-0908 & 1.27645 & -14.5884 & 3.86 & -9.83892 & -15.9235 & -34.0112 \\
\hline J1623-2631 & 0.011076 & -18.1733 & 1.8 & -15.4854 & -17.4468 & -35.2031 \\
\hline $\mathrm{J} 1623-4256$ & 0.36459 & -14.9914 & 21.85 & -10.7861 & -15.7824 & -34.6229 \\
\hline J1623-4949 & 0.725732 & -13.3757 & 3.76 & -8.87149 & -14.4656 & -32.5419 \\
\hline J1624-4411 & 0.233164 & -15.0975 & 3.64 & -11.0863 & -15.6943 & -33.7564 \\
\hline
\end{tabular}


Table 1 continued

\begin{tabular}{|c|c|c|c|c|c|c|}
\hline Pulsar & $P(\mathrm{~s})$ & $\log \dot{P}$ & $d(\mathrm{kpc})$ & $\log \varepsilon$ & $\log \eta$ & $\log h$ \\
\hline J1624-4613 & 0.871243 & -16.2168 & 5.14 & -11.6332 & -17.3861 & -35.5981 \\
\hline J1624-4721 & 0.448723 & -14.382 & 6.11 & -10.0865 & -15.2631 & -33.5502 \\
\hline $\mathrm{J} 1625-4048$ & 2.35528 & -15.3536 & 5.18 & -10.3381 & -16.9548 & -35.1702 \\
\hline J1625-4904 & 0.460339 & -13.7747 & 7.94 & -9.46816 & -14.6669 & -33.0678 \\
\hline $\mathrm{J} 1625-4913$ & 0.355856 & -14.1772 & 8.21 & -9.98245 & -14.9576 & -33.373 \\
\hline J1626-4537 & 0.370141 & -14.082 & 5.41 & -9.87015 & -14.8795 & -33.1137 \\
\hline J1626-4807 & 0.293928 & -13.757 & 10.25 & -9.64527 & -14.4544 & -32.9661 \\
\hline J1626-6621 & 0.450868 & -15.1158 & 4.42 & -10.8183 & -15.999 & -34.1454 \\
\hline $\mathrm{J} 1627+1419$ & 0.490857 & -15.4056 & 2.84 & -11.0712 & -16.3257 & -34.2801 \\
\hline J1627-4706 & 0.140746 & -14.762 & 7.15 & -10.9701 & -15.1395 & -33.4949 \\
\hline $\mathrm{J} 1627-4845$ & 0.612331 & -14.4389 & 6.86 & -10.0085 & -15.455 & -33.7924 \\
\hline $\mathrm{J} 1627-5547$ & 0.352464 & -15.1319 & 5.66 & -10.9414 & -15.9082 & -34.1621 \\
\hline J1627-5936 & 0.354234 & -17.0969 & 3.67 & -12.9042 & -17.8753 & -35.9411 \\
\hline $\mathrm{J} 1628-4804$ & 0.865971 & -14.9066 & 11.21 & -10.3256 & -16.0732 & -34.6239 \\
\hline J1628-4828 & 4.13754 & -13.757 & 14.29 & -8.49677 & -15.6029 & -34.2589 \\
\hline J1629-3636 & 2.98819 & -14.1549 & 3.74 & -9.03604 & -15.8595 & -33.9334 \\
\hline J1629-6902 & 0.006001 & -20 & 1.36 & -17.5783 & -19.0074 & -36.642 \\
\hline $\mathrm{J} 1630-4719$ & 0.559071 & -13.8477 & 6.7 & -9.45679 & -14.8243 & -33.1515 \\
\hline $\mathrm{J} 1630-4733$ & 0.575971 & -13.6517 & 6.53 & -9.24784 & -14.6412 & -32.9572 \\
\hline $\mathrm{J} 1631-1612$ & 0.677684 & -14.9586 & 1.85 & -10.4841 & -16.0188 & -33.787 \\
\hline $\mathrm{J} 1631-4155$ & 0.551241 & -17.055 & 8.51 & -12.6702 & -18.0255 & -36.4565 \\
\hline $\mathrm{J} 1632-1013$ & 0.717637 & -16.1805 & 4.22 & -11.6811 & -17.2655 & -35.3919 \\
\hline $\mathrm{J} 1632-4509$ & 1.04681 & -13.8268 & 9.13 & -9.16349 & -15.0758 & -33.5374 \\
\hline $\mathrm{J} 1632-4621$ & 1.70915 & -13.1192 & 8.42 & -8.24295 & -14.5811 & -33.0075 \\
\hline $\mathrm{J} 1632-4757$ & 0.228564 & -13.821 & 6.96 & -9.81856 & -14.4092 & -32.7528 \\
\hline $\mathrm{J} 1632-4818$ & 0.813453 & -12.1871 & 8.54 & -7.6333 & -13.3266 & -31.7591 \\
\hline J1633-2009 & 0.935557 & -14.9706 & 2.76 & -10.3561 & -16.1708 & -34.1128 \\
\hline $\mathrm{J} 1633-4453$ & 0.436507 & -14.2076 & 11.93 & -9.92416 & -15.0767 & -33.6544 \\
\hline $\mathrm{J} 1633-4805$ & 0.71083 & -13.1141 & 11.9 & -8.61886 & -14.195 & -32.7716 \\
\hline J1633-5015 & 0.352142 & -14.4214 & 7.02 & -10.2312 & -15.1972 & -33.5446 \\
\hline J1634-5107 & 0.507356 & -14.8041 & 9.3 & -10.4553 & -15.7386 & -34.2081 \\
\hline J1634-5640 & 0.224201 & -16.3872 & 5.71 & -12.3931 & -16.967 & -35.2247 \\
\hline $\mathrm{J} 1635-1511$ & 1.17939 & -15.6345 & 4.89 & -10.9194 & -16.9353 & -35.1257 \\
\hline $\mathrm{J} 1635+2418$ & 0.490507 & -15.9245 & 2.27 & -11.5904 & -16.8442 & -34.7013 \\
\hline $\mathrm{J} 1635-4513$ & 1.59475 & -14.4437 & 6.96 & -9.59755 & -15.8755 & -34.2192 \\
\hline J1635-4944 & 0.671964 & -14.056 & 8.85 & -9.58521 & -15.1125 & -33.5605 \\
\hline J1635-5954 & 0.529121 & -14.8633 & 7.26 & -10.4963 & -15.816 & -34.178 \\
\hline $\mathrm{J} 1636-4440$ & 0.206649 & -13.3307 & 9.33 & -9.372 & -13.8751 & -32.346 \\
\hline $\mathrm{J} 1636-4803$ & 1.20464 & -13.684 & 6.53 & -8.95972 & -14.994 & -33.31 \\
\hline J1636-4933 & 0.430367 & -14.821 & 11.16 & -10.5437 & -15.684 & -34.2327 \\
\hline $\mathrm{J} 1637-4335$ & 0.771367 & -14.4413 & 30 & -9.91058 & -15.5577 & -34.5359 \\
\hline $\mathrm{J} 1637-4450$ & 0.25287 & -15.2403 & 8.2 & -11.194 & -15.8724 & -34.2873 \\
\hline $\mathrm{J} 1637-4553$ & 0.118771 & -14.4962 & 3.83 & -10.778 & -14.8001 & -32.8843 \\
\hline $\mathrm{J} 1637-4642$ & 0.154027 & -13.2277 & 5.77 & -9.39663 & -13.6444 & -31.9067 \\
\hline $\mathrm{J} 1637-4721$ & 1.16574 & -14.3526 & 5.94 & -9.64256 & -15.6484 & -33.9232 \\
\hline $\mathrm{J} 1637-4816$ & 0.837365 & -14.2343 & 10.01 & -9.66796 & -15.3864 & -33.8879 \\
\hline $\mathrm{J} 1638-3815$ & 0.698261 & -16.1135 & 17.54 & -11.626 & -17.1867 & -35.9318 \\
\hline
\end{tabular}


Table 1 continued

\begin{tabular}{|c|c|c|c|c|c|c|}
\hline Pulsar & $P(\mathrm{~s})$ & $\log \dot{P}$ & $d(\mathrm{kpc})$ & $\log \varepsilon$ & $\log \eta$ & $\log h$ \\
\hline $\mathrm{J} 1638-3951$ & 0.77113 & -15.2291 & 10.96 & -10.6986 & -16.3454 & -34.8863 \\
\hline J1638-4344 & 1.12194 & -16.6021 & 5.09 & -11.9086 & -17.8812 & -36.089 \\
\hline $\mathrm{J} 1638-4417$ & 0.117802 & -14.7932 & 8.46 & -11.0786 & -15.0935 & -33.5219 \\
\hline $\mathrm{J} 1638-4608$ & 0.278137 & -13.2882 & 5.85 & -9.20048 & -13.9616 & -32.2298 \\
\hline $\mathrm{J} 1638-4725$ & 0.763933 & -14.3197 & 6.87 & -9.79316 & -15.4319 & -33.7699 \\
\hline J1638-5226 & 0.340503 & -14.5768 & 4.88 & -10.4012 & -15.338 & -33.5275 \\
\hline J1639-4359 & 0.587559 & -16.8239 & 5.23 & -12.4114 & -17.8221 & -36.0417 \\
\hline J1639-4604 & 0.529116 & -14.2381 & 4.64 & -9.87107 & -15.1908 & -33.3583 \\
\hline $\mathrm{J} 1640+2224$ & 0.003163 & -20.5482 & 1.45 & -18.4047 & -19.2775 & -36.9399 \\
\hline $\mathrm{J} 1640-4631$ & 0.206443 & -12.0106 & 12.75 & -8.0523 & -12.5545 & -31.1611 \\
\hline $\mathrm{J} 1640-4648$ & 0.178352 & -15.0937 & 6.1 & -11.1989 & -15.5741 & -33.8605 \\
\hline $\mathrm{J} 1640-4715$ & 0.517405 & -13.3768 & 7.25 & -9.01947 & -14.3197 & -32.6811 \\
\hline J1640-4951 & 0.739099 & -15.4763 & 10.66 & -10.9641 & -16.5741 & -35.1029 \\
\hline $\mathrm{J} 1641-2347$ & 1.09101 & -16.3862 & 1.33 & -11.7049 & -17.6531 & -35.278 \\
\hline J1643-1224 & 0.004622 & -19.7328 & 0.74 & -17.4245 & -18.6268 & -35.9971 \\
\hline $\mathrm{J} 1643-4505$ & 0.237383 & -13.4976 & 6.3 & -9.47867 & -14.1022 & -32.4026 \\
\hline $\mathrm{J} 1643-4522$ & 1.3479 & -14.082 & 6.19 & -9.30886 & -15.4408 & -33.7335 \\
\hline $\mathrm{J} 1643-4550$ & 0.717508 & -13.5229 & 5.88 & -9.0236 & -14.6079 & -32.8783 \\
\hline J1644-4559 & 0.45506 & -13.6968 & 4.5 & -9.39528 & -14.584 & -32.7383 \\
\hline $\mathrm{J} 1644-4657$ & 0.125962 & -15.1249 & 9.58 & -11.3812 & -15.4543 & -33.9368 \\
\hline $\mathrm{J} 1645-0317$ & 0.38769 & -14.7496 & 2.61 & -10.5176 & -15.5672 & -33.4849 \\
\hline $\mathrm{J} 1645+1012$ & 0.410861 & -16.0899 & 3.27 & -11.8328 & -16.9328 & -34.9484 \\
\hline J1646-4308 & 0.84068 & -15.9586 & 10.96 & -11.3905 & -17.1124 & -35.6533 \\
\hline J1646-4346 & 0.231603 & -12.9469 & 6.86 & -8.93872 & -13.5408 & -31.8782 \\
\hline $\mathrm{J} 1646-5123$ & 0.530075 & -14.6778 & 10.92 & -10.31 & -15.6313 & -34.1705 \\
\hline J1646-6831 & 1.78561 & -14.7696 & 1.8 & -9.87431 & -16.2505 & -34.0068 \\
\hline $\mathrm{J} 1647-3607$ & 0.212316 & -15.8894 & 12.9 & -11.919 & -16.4455 & -35.0572 \\
\hline $\mathrm{J} 1648-3256$ & 0.719455 & -14.4522 & 5.3 & -9.95177 & -15.5384 & -33.7637 \\
\hline $\mathrm{J} 1648-4458$ & 0.629632 & -14.7328 & 9.89 & -10.2903 & -15.7611 & -34.2573 \\
\hline J1648-4611 & 0.16495 & -13.6253 & 5.71 & -9.76445 & -14.0718 & -32.3294 \\
\hline J1648-6044 & 0.583765 & -15.3675 & 5.66 & -10.9579 & -16.3629 & -34.6168 \\
\hline $\mathrm{J} 1649+2533$ & 1.01526 & -15.2526 & 2.91 & -10.6026 & -16.4883 & -34.4533 \\
\hline $\mathrm{J} 1649-3805$ & 0.262026 & -16.4353 & 6.62 & -12.3735 & -17.0828 & -35.4047 \\
\hline J1649-3935 & 0.77091 & -16.4078 & 9.04 & -11.8774 & -17.524 & -35.9812 \\
\hline J1649-4349 & 0.870712 & -16.3565 & 5.56 & -11.7732 & -17.5256 & -35.7717 \\
\hline $\mathrm{J} 1649-4653$ & 0.557019 & -13.3036 & 5.63 & -8.91432 & -14.2787 & -32.5302 \\
\hline J1649-4729 & 0.297692 & -14.1838 & 12.69 & -10.0665 & -14.8867 & -33.4912 \\
\hline $\mathrm{J} 1649-5553$ & 0.613571 & -14.7696 & 14.07 & -10.3382 & -15.7866 & -34.4359 \\
\hline $\mathrm{J} 1650-1654$ & 1.74955 & -14.4949 & 2.15 & -9.60847 & -15.9669 & -33.8004 \\
\hline $\mathrm{J} 1650-4126$ & 0.308918 & -16.7033 & 5.06 & -12.57 & -17.4223 & -35.6275 \\
\hline $\mathrm{J} 1650-4341$ & 0.309398 & -16.7773 & 8.11 & -12.6433 & -17.4969 & -35.907 \\
\hline $\mathrm{J} 1650-4502$ & 0.38087 & -13.7932 & 5.09 & -9.56894 & -14.6031 & -32.8109 \\
\hline J1650-4921 & 0.156399 & -14.7399 & 5.83 & -10.9022 & -15.1633 & -33.43 \\
\hline J1651-1709 & 0.973394 & -14.5171 & 1.62 & -9.88539 & -15.7346 & -33.4451 \\
\hline $\mathrm{J} 1651-4246$ & 0.844081 & -14.3179 & 5.2 & -9.74802 & -15.4734 & -33.6904 \\
\hline $\mathrm{J} 1651-4519$ & 0.517443 & -14.0867 & 7.29 & -9.7294 & -15.0297 & -33.3935 \\
\hline $\mathrm{J} 1651-5222$ & 0.635056 & -14.7423 & 6.39 & -10.2961 & -15.7743 & -34.0808 \\
\hline
\end{tabular}


Table 1 continued

\begin{tabular}{|c|c|c|c|c|c|c|}
\hline Pulsar & $P(\mathrm{~s})$ & $\log \dot{P}$ & $d(\mathrm{kpc})$ & $\log \varepsilon$ & $\log \eta$ & $\log h$ \\
\hline $\mathrm{J} 1651-5255$ & 0.890534 & -14.684 & 5.99 & -10.0909 & -15.8628 & -34.1413 \\
\hline $\mathrm{J} 1651-7642$ & 1.75531 & -14.8665 & 5.16 & -9.97865 & -16.34 & -34.5537 \\
\hline $\mathrm{J} 1652-1400$ & 0.305447 & -16.7545 & 3.1 & -12.6261 & -17.4686 & -35.461 \\
\hline $\mathrm{J} 1652-2404$ & 1.70374 & -14.5003 & 3.1 & -9.62546 & -15.9609 & -33.9533 \\
\hline $\mathrm{J} 1652+2651$ & 0.915803 & -15.1844 & 2.93 & -10.5792 & -16.3754 & -34.3433 \\
\hline J1652-4406 & 7.70718 & -14.0223 & 8.8 & -8.49193 & -16.1383 & -34.5839 \\
\hline $\mathrm{J} 1653-2054$ & 0.004129 & -19.9508 & 2.64 & -17.6915 & -18.7958 & -36.7184 \\
\hline $\mathrm{J} 1653-3838$ & 0.305037 & -14.5544 & 5.12 & -10.4266 & -15.2679 & -33.4782 \\
\hline $\mathrm{J} 1653-4030$ & 1.01937 & -15.3605 & 10.37 & -10.7087 & -16.598 & -35.1148 \\
\hline $\mathrm{J} 1653-4249$ & 0.612558 & -14.3179 & 5.64 & -9.88726 & -15.3342 & -33.5865 \\
\hline $\mathrm{J} 1653-4315$ & 0.41928 & -16.8239 & 5.13 & -12.558 & -17.6756 & -35.8867 \\
\hline $\mathrm{J} 1653-4854$ & 3.05951 & -14.4622 & 13.76 & -9.33308 & -16.177 & -34.8167 \\
\hline $\mathrm{J} 1654-2713$ & 0.791822 & -15.7747 & 4.08 & -11.2326 & -16.9025 & -35.0142 \\
\hline $\mathrm{J} 1654-3710$ & 0.939165 & -15.1337 & 13.15 & -10.5175 & -16.3356 & -34.9556 \\
\hline $\mathrm{J} 1654-4140$ & 1.27395 & -15.8861 & 5.15 & -11.1375 & -17.2204 & -35.4332 \\
\hline $\mathrm{J} 1654-4245$ & 1.10156 & -13.2907 & 11.84 & -8.60527 & -14.5619 & -33.1363 \\
\hline $\mathrm{J} 1655-3048$ & 0.542936 & -16.4365 & 8.57 & -12.0583 & -17.4004 & -35.8345 \\
\hline $\mathrm{J} 1655-3844$ & 1.19344 & -14.699 & 11.94 & -9.97872 & -16.0049 & -34.583 \\
\hline $\mathrm{J} 1656-3621$ & 0.730134 & -14.8928 & 7.6 & -10.3859 & -15.9853 & -34.3672 \\
\hline J1657-4432 & 0.609607 & -14.0862 & 5.58 & -9.65768 & -15.1004 & -33.3481 \\
\hline $\mathrm{J} 1658-4306$ & 1.16645 & -13.3686 & 9.59 & -8.65824 & -14.6646 & -33.1474 \\
\hline J1658-4958 & 0.416874 & -14.4134 & 6.26 & -10.15 & -15.2626 & -33.5602 \\
\hline J1658-5324 & 0.002439 & -19.9508 & 1.24 & -17.9201 & -18.5671 & -36.1616 \\
\hline J1659-1305 & 0.640958 & -15.209 & 4.85 & -10.7587 & -16.245 & -34.4318 \\
\hline J1659-4316 & 0.474381 & -15.767 & 7.93 & -11.4474 & -16.6723 & -35.0726 \\
\hline J1659-4439 & 0.353293 & -16.6021 & 9.18 & -12.4105 & -17.3793 & -35.8432 \\
\hline $\mathrm{J} 1700-3312$ & 1.35831 & -14.327 & 5.64 & -9.55053 & -15.6891 & -33.9415 \\
\hline $\mathrm{J} 1700-3611$ & 1.49409 & -14.3645 & 6.48 & -9.54669 & -15.768 & -34.0807 \\
\hline J1700-3919 & 0.560504 & -17.2984 & 6.34 & -12.9064 & -18.2762 & -36.5793 \\
\hline $\mathrm{J} 1700-4012$ & 0.283792 & -16.0039 & 5.77 & -11.9075 & -16.6861 & -34.9483 \\
\hline $\mathrm{J} 1700-4422$ & 0.755535 & -16.3979 & 6.44 & -11.8762 & -17.5053 & -35.8153 \\
\hline J1700-4939 & 0.578363 & -14.9666 & 14.53 & -10.5609 & -15.9579 & -34.6212 \\
\hline $\mathrm{J} 1701-3130$ & 0.291341 & -16.2518 & 4.53 & -12.144 & -16.9454 & -35.1025 \\
\hline J1701-3726 & 2.45461 & -13.9547 & 7.81 & -8.92124 & -15.5738 & -33.9675 \\
\hline $\mathrm{J} 1701-4533$ & 0.322909 & -15.2848 & 17.39 & -11.1323 & -16.0231 & -34.7644 \\
\hline $\mathrm{J} 1701-4958$ & 0.804304 & -15.9586 & 9.68 & -11.4097 & -17.0932 & -35.5801 \\
\hline J1702-3932 & 0.390328 & -15.4225 & 9.69 & -11.1876 & -16.2431 & -34.7305 \\
\hline $\mathrm{J} 1702-4128$ & 0.182136 & -13.2815 & 5.18 & -9.37765 & -13.771 & -31.9864 \\
\hline $\mathrm{J} 1702-4217$ & 0.227565 & -16.9431 & 7.49 & -12.9425 & -17.5293 & -35.9049 \\
\hline J1702-4306 & 0.215507 & -14.0092 & 7.12 & -10.0323 & -14.5718 & -32.9254 \\
\hline $\mathrm{J} 1702-4310$ & 0.240524 & -12.6498 & 5.44 & -8.62514 & -13.2601 & -31.4967 \\
\hline $\mathrm{J} 1702-4428$ & 2.12351 & -14.4815 & 7.05 & -9.51098 & -16.0377 & -34.3869 \\
\hline $\mathrm{J} 1703-1846$ & 0.804341 & -14.762 & 2.05 & -10.2131 & -15.8965 & -33.7094 \\
\hline $\mathrm{J} 1703-3241$ & 1.21178 & -15.1805 & 3.31 & -10.4536 & -16.493 & -34.5139 \\
\hline $\mathrm{J} 1703-4442$ & 1.74729 & -13.8447 & 5.34 & -8.95885 & -15.3162 & -33.5448 \\
\hline $\mathrm{J} 1703-4851$ & 1.3964 & -14.2941 & 4.5 & -9.50567 & -15.6683 & -33.8226 \\
\hline $\mathrm{J} 1704-6016$ & 0.306323 & -15.041 & 1.89 & -10.9113 & -15.7563 & -33.5338 \\
\hline
\end{tabular}


Table 1 continued

\begin{tabular}{|c|c|c|c|c|c|c|}
\hline Pulsar & $P(\mathrm{~s})$ & $\log \dot{P}$ & $d(\mathrm{kpc})$ & $\log \varepsilon$ & $\log \eta$ & $\log h$ \\
\hline J1705-1906 & 0.298987 & -14.383 & 1.18 & -10.2639 & -15.0878 & -32.6607 \\
\hline $\mathrm{J} 1705-3423$ & 0.255426 & -14.9666 & 3.75 & -10.9159 & -15.603 & -33.6781 \\
\hline J1705-3936 & 0.854482 & -13.7144 & 8.46 & -9.13929 & -14.8753 & -33.3037 \\
\hline $\mathrm{J} 1705-3950$ & 0.318941 & -13.2175 & 3.86 & -9.07036 & -13.9504 & -32.038 \\
\hline $\mathrm{J} 1705-4108$ & 0.861067 & -13.4597 & 11.87 & -8.88118 & -14.6239 & -33.1994 \\
\hline $\mathrm{J} 1705-4331$ & 0.222561 & -16.1475 & 3.76 & -12.1566 & -16.7241 & -34.8004 \\
\hline $\mathrm{J} 1705-6135$ & 0.808546 & -16.2218 & 6.31 & -11.6707 & -17.3587 & -35.6598 \\
\hline J1706-3839 & 0.586287 & -14.5229 & 12.2 & -10.1113 & -15.5201 & -34.1076 \\
\hline $\mathrm{J} 1706-4310$ & 0.616979 & -14.1864 & 13.43 & -9.7527 & -15.2058 & -33.835 \\
\hline J1706-6118 & 0.361921 & -15.5346 & 3.64 & -11.3326 & -16.3224 & -34.3845 \\
\hline $\mathrm{J} 1707-4053$ & 0.581017 & -14.7167 & 4 & -10.3091 & -15.71 & -33.8132 \\
\hline $\mathrm{J} 1707-4341$ & 0.890594 & -14.2441 & 7.99 & -9.65099 & -15.423 & -33.8266 \\
\hline J1707-4417 & 5.76378 & -13.9355 & 9.17 & -8.53138 & -15.9254 & -34.3888 \\
\hline J1707-4729 & 0.266474 & -14.8069 & 10.64 & -10.7378 & -15.4617 & -33.9897 \\
\hline $\mathrm{J} 1708-3426$ & 0.692113 & -14.3768 & 4.78 & -9.89312 & -15.4461 & -33.6266 \\
\hline J1708-3506 & 0.004505 & -19.9431 & 3.5 & -17.6459 & -18.8259 & -36.8711 \\
\hline J1708-3641 & 0.587567 & -15.8697 & 6.02 & -11.4572 & -16.8679 & -35.1485 \\
\hline $\mathrm{J} 1708-3827$ & 1.22578 & -14.0655 & 17.55 & -9.33364 & -15.3831 & -34.1284 \\
\hline $\mathrm{J} 1708-4008$ & 11.0063 & -10.7077 & 3.8 & -5.02265 & -12.9785 & -31.0594 \\
\hline J1708-4522 & 1.29784 & -14.5834 & 30 & -9.82669 & -15.9257 & -34.9039 \\
\hline J1708-7539 & 1.19102 & -14.7258 & 2.01 & -10.0065 & -16.0309 & -33.8352 \\
\hline $\mathrm{J} 1709-1640$ & 0.653054 & -14.2 & 2.61 & -9.74157 & -15.2441 & -33.1618 \\
\hline $\mathrm{J} 1709+2313$ & 0.004631 & -20.4401 & 1.83 & -18.131 & -19.3349 & -37.0984 \\
\hline J1709-3626 & 0.447857 & -14.644 & 8.97 & -10.3494 & -15.5243 & -33.9781 \\
\hline J1709-3841 & 0.586986 & -14.1046 & 5.17 & -9.6925 & -15.1024 & -33.3169 \\
\hline $\mathrm{J} 1709-4342$ & 1.7359 & -15.0969 & 5.49 & -10.2139 & -16.5656 & -34.8062 \\
\hline J1709-4401 & 0.865235 & -14.1325 & 4.98 & -9.55195 & -15.2988 & -33.4971 \\
\hline $\mathrm{J} 1709-4429$ & 0.102459 & -13.0315 & 2.6 & -9.37751 & -13.2712 & -31.1872 \\
\hline J1710-2616 & 0.954158 & -16.699 & 4.26 & -12.0759 & -17.9077 & -36.0382 \\
\hline $\mathrm{J} 1710-4148$ & 0.286561 & -15.9914 & 6.9 & -11.8907 & -16.6778 & -35.0177 \\
\hline J1711-1509 & 0.868804 & -14.9586 & 2.94 & -10.3762 & -16.1267 & -34.0961 \\
\hline $\mathrm{J} 1711-3826$ & 0.465365 & -14.1302 & 5.2 & -9.81894 & -15.0271 & -33.2442 \\
\hline $\mathrm{J} 1711-4322$ & 0.102618 & -16.5735 & 4.17 & -12.9188 & -16.8139 & -34.9351 \\
\hline $\mathrm{J} 1711-5350$ & 0.899218 & -13.8097 & 4.24 & -9.21235 & -14.9927 & -33.1211 \\
\hline $\mathrm{J} 1712-2715$ & 0.25536 & -14.8928 & 3.13 & -10.8422 & -15.5291 & -33.5257 \\
\hline $\mathrm{J} 1713+0747$ & 0.00457 & -20.0691 & 1.18 & -17.7657 & -18.9581 & -36.5311 \\
\hline J1713-3844 & 1.60011 & -12.752 & 6.5 & -7.90442 & -14.1853 & -32.4993 \\
\hline $\mathrm{J} 1714-1054$ & 0.696279 & -16.2306 & 2.65 & -11.7444 & -17.3026 & -35.2269 \\
\hline $\mathrm{J} 1714-3810$ & 3.82494 & -10.2306 & 13.2 & -5.00455 & -12.0424 & -30.664 \\
\hline $\mathrm{J} 1715-3247$ & 1.26021 & -16.0362 & 3.91 & -11.2923 & -17.3658 & -35.459 \\
\hline $\mathrm{J} 1715-3700$ & 0.779628 & -15.8356 & 6.08 & -11.3003 & -16.9567 & -35.2416 \\
\hline J1715-3859 & 0.928108 & -14.3565 & 10.53 & -9.7455 & -15.5533 & -34.0768 \\
\hline J1715-3903 & 0.278481 & -13.4237 & 4.8 & -9.33541 & -14.0976 & -32.2799 \\
\hline $\mathrm{J} 1715-4034$ & 2.07215 & -14.5214 & 4.62 & -9.56156 & -16.067 & -34.2327 \\
\hline $\mathrm{J} 1715-4254$ & 0.573745 & -15.0575 & 12.58 & -10.6553 & -16.0454 & -34.6461 \\
\hline J1716-3720 & 0.630314 & -13.7447 & 9.46 & -9.30172 & -14.7734 & -33.2504 \\
\hline $\mathrm{J} 1716-4005$ & 0.311813 & -14.5421 & 6.33 & -10.4048 & -15.2652 & -33.5676 \\
\hline
\end{tabular}


Table 1 continued

\begin{tabular}{|c|c|c|c|c|c|c|}
\hline Pulsar & $P(\mathrm{~s})$ & $\log \dot{P}$ & $d(\mathrm{kpc})$ & $\log \varepsilon$ & $\log \eta$ & $\log h$ \\
\hline $\mathrm{J} 1716-4111$ & 1.03607 & -14.5406 & 4.79 & -9.88177 & -15.7851 & -33.9665 \\
\hline J1716-4711 & 0.555824 & -15.0794 & 19.58 & -10.691 & -16.0534 & -34.8463 \\
\hline $\mathrm{J} 1717-3425$ & 0.656299 & -14.0088 & 22.21 & -9.54822 & -15.055 & -33.9026 \\
\hline $\mathrm{J} 1717-3737$ & 0.682419 & -14.2765 & 6.31 & -9.79904 & -15.3397 & -33.6408 \\
\hline $\mathrm{J} 1717-3847$ & 1.1495 & -15.0862 & 9.52 & -10.3822 & -16.3758 & -34.8555 \\
\hline $\mathrm{J} 1717-3953$ & 1.08552 & -16.4815 & 6.88 & -11.8024 & -17.7463 & -36.0849 \\
\hline $\mathrm{J} 1717-4043$ & 0.397857 & -13.9136 & 9.03 & -9.67046 & -14.7425 & -33.1993 \\
\hline $\mathrm{J} 1717-40435$ & 0.349929 & -14.757 & 11.41 & -10.5695 & -15.5301 & -34.0884 \\
\hline $\mathrm{J} 1717-4054$ & 0.887719 & -14.4342 & 5.47 & -9.84242 & -15.6116 & -33.8506 \\
\hline $\mathrm{J} 1717-5800$ & 0.321793 & -15.7077 & 8.85 & -11.5567 & -16.4445 & -34.8925 \\
\hline J1718-3714 & 1.28938 & -13.5817 & 10.87 & -8.82787 & -14.9212 & -33.4585 \\
\hline $\mathrm{J} 1718-3718$ & 3.37857 & -11.7932 & 5.08 & -6.62099 & -13.5511 & -31.758 \\
\hline $\mathrm{J} 1718-3825$ & 0.07467 & -13.8794 & 4.24 & -10.3628 & -13.9817 & -32.1101 \\
\hline $\mathrm{J} 1718-4539$ & 0.590473 & -14.1244 & 10.82 & -9.70971 & -15.1247 & -33.66 \\
\hline $\mathrm{J} 1719-1438$ & 0.00579 & -20.0947 & 1.64 & -17.6886 & -19.0866 & -36.8025 \\
\hline $\mathrm{J} 1719-4006$ & 0.189094 & -14.7773 & 6.28 & -10.8572 & -15.2831 & -33.5821 \\
\hline $\mathrm{J} 1719-4302$ & 0.235475 & -15.4067 & 9.42 & -11.3913 & -16.0078 & -34.4829 \\
\hline $\mathrm{J} 1720-0212$ & 0.477715 & -16.082 & 5.41 & -11.7593 & -16.9903 & -35.2245 \\
\hline $\mathrm{J} 1720-1633$ & 1.5656 & -14.2366 & 1.76 & -9.39844 & -15.6604 & -33.407 \\
\hline $\mathrm{J} 1720+2150$ & 1.61566 & -15.1308 & 3.59 & -10.279 & -16.5683 & -34.6244 \\
\hline J1720-2446 & 0.874265 & -15.2269 & 3.44 & -10.6418 & -16.3977 & -34.4354 \\
\hline J1720-2933 & 0.620448 & -15.1273 & 1.43 & -10.6911 & -16.1491 & -33.8055 \\
\hline J1720-3659 & 0.351125 & -16.4855 & 5.11 & -12.2965 & -17.2601 & -35.4695 \\
\hline J1721-1936 & 1.00404 & -14.7905 & 8.6 & -10.1453 & -16.0214 & -34.4569 \\
\hline J1721-1939 & 0.40404 & -15.8928 & 4.64 & -11.6429 & -16.7284 & -34.8959 \\
\hline $\mathrm{J} 1721-2457$ & 0.003497 & -20.2565 & 1.56 & -18.0693 & -19.0293 & -36.7235 \\
\hline $\mathrm{J} 1721-3532$ & 0.280424 & -13.5986 & 4.6 & -9.50733 & -14.2756 & -32.4394 \\
\hline J1722-3207 & 0.477158 & -15.1898 & 3.18 & -10.8677 & -16.0976 & -34.1011 \\
\hline $\mathrm{J} 1722-3632$ & 0.399183 & -14.3507 & 5.3 & -10.106 & -15.181 & -33.4063 \\
\hline $\mathrm{J} 1722-3712$ & 0.236173 & -13.9626 & 2.51 & -9.94589 & -14.565 & -32.4657 \\
\hline $\mathrm{J} 1722-4400$ & 0.218554 & -15.4271 & 7.27 & -11.4441 & -15.9958 & -34.3584 \\
\hline $\mathrm{J} 1723-2837$ & 0.001856 & -20.1226 & 1 & -18.2106 & -18.6204 & -36.1214 \\
\hline $\mathrm{J} 1723-2852$ & 0.625034 & -15.0458 & 4.63 & -10.6064 & -16.0708 & -34.2374 \\
\hline $\mathrm{J} 1723-3659$ & 0.202722 & -14.0964 & 4.28 & -10.146 & -14.6324 & -32.7649 \\
\hline $\mathrm{J} 1724-3149$ & 0.948237 & -14.1397 & 10.5 & -9.51929 & -15.3457 & -33.868 \\
\hline $\mathrm{J} 1724-3505$ & 1.22171 & -13.6757 & 12.01 & -8.9453 & -14.9918 & -33.5724 \\
\hline $\mathrm{J} 1724-4500$ & 1.30911 & -14.4547 & 6.26 & -9.69426 & -15.8008 & -34.0985 \\
\hline $\mathrm{J} 1725-0732$ & 0.239919 & -15.3665 & 3.43 & -11.343 & -15.9757 & -34.0121 \\
\hline $\mathrm{J} 1725-2852$ & 1.25779 & -14.7011 & 4.09 & -9.95809 & -16.0299 & -34.1427 \\
\hline $\mathrm{J} 1725-3546$ & 1.03247 & -13.8239 & 10.2 & -9.16658 & -15.0669 & -33.5766 \\
\hline $\mathrm{J} 1725-3848$ & 2.06239 & -13.6383 & 4.49 & -8.68045 & -15.1818 & -33.3351 \\
\hline $\mathrm{J} 1725-3853$ & 0.004792 & -19.301 & 3.44 & -16.9771 & -18.2107 & -36.2483 \\
\hline $\mathrm{J} 1725-4043$ & 1.46507 & -14.5544 & 4.8 & -9.74508 & -15.9494 & -34.1317 \\
\hline $\mathrm{J} 1726-3530$ & 1.11013 & -11.9136 & 9.97 & -7.22481 & -13.1882 & -31.6879 \\
\hline J1726-3635 & 0.287432 & -14.8416 & 7.36 & -10.7396 & -15.5293 & -33.8973 \\
\hline J1726-4006 & 0.882778 & -14.4776 & 6.19 & -9.88825 & -15.6526 & -33.9453 \\
\hline J1727-2739 & 1.2931 & -14.9586 & 3.75 & -10.2035 & -16.2994 & -34.3745 \\
\hline
\end{tabular}


Table 1 continued

\begin{tabular}{|c|c|c|c|c|c|c|}
\hline Pulsar & $P(\mathrm{~s})$ & $\log \dot{P}$ & $d(\mathrm{kpc})$ & $\log \varepsilon$ & $\log \eta$ & $\log h$ \\
\hline $\mathrm{J} 1727-2946$ & 0.027083 & -18.6091 & 1.62 & -15.5329 & -18.2709 & -35.9815 \\
\hline $\mathrm{J} 1728-0007$ & 0.386004 & -14.9508 & 2.44 & -10.7207 & -15.7665 & -33.655 \\
\hline J1728-3733 & 0.615538 & -16.1427 & 4.94 & -11.71 & -17.1611 & -35.3559 \\
\hline $\mathrm{J} 1728-4028$ & 0.866343 & -15.6326 & 5.51 & -11.0515 & -16.7995 & -35.0417 \\
\hline $\mathrm{J} 1729-2117$ & 0.066293 & -18.7645 & 1.42 & -15.2996 & -18.8151 & -36.4684 \\
\hline $\mathrm{J} 1730-2304$ & 0.008123 & -19.6946 & 0.62 & -17.1415 & -18.8335 & -36.127 \\
\hline $\mathrm{J} 1730-2900$ & 1.53843 & -14.0531 & 6.85 & -9.22253 & -15.4693 & -33.806 \\
\hline $\mathrm{J} 1730-3350$ & 0.13946 & -13.0716 & 4.26 & -9.2837 & -13.4452 & -31.5757 \\
\hline $\mathrm{J} 1730-3353$ & 3.27024 & -13.6576 & 4.23 & -8.49955 & -15.4013 & -33.5287 \\
\hline $\mathrm{J} 1731-1847$ & 0.002345 & -19.5952 & 4.03 & -17.5816 & -18.1945 & -36.3008 \\
\hline $\mathrm{J} 1731-3123$ & 0.753048 & -14.7747 & 5.32 & -10.2544 & -15.8807 & -34.1076 \\
\hline $\mathrm{J} 1731-3322$ & 0.544671 & -13.5513 & 11.98 & -9.17171 & -14.5166 & -33.0961 \\
\hline J1731-4744 & 0.829829 & -12.7852 & 4.98 & -8.22271 & -13.9333 & -32.1316 \\
\hline J1732-1930 & 0.48377 & -15.7399 & 2.21 & -11.4118 & -16.6537 & -34.4992 \\
\hline $\mathrm{J} 1732-3131$ & 0.196543 & -13.5528 & 0.8 & -9.61593 & -14.0754 & -31.4796 \\
\hline J1732-3426 & 0.332887 & -15.3958 & 6.38 & -11.23 & -16.1472 & -34.4531 \\
\hline J1732-3729 & 2.184 & -14.8861 & 6.2 & -9.90335 & -16.4545 & -34.7479 \\
\hline $\mathrm{J} 1732-4128$ & 0.627981 & -13.8928 & 5.94 & -9.45139 & -14.9199 & -33.1947 \\
\hline J1732-4156 & 0.323434 & -15.1798 & 8.78 & -11.0266 & -15.9187 & -34.3633 \\
\hline J1732-5049 & 0.005313 & -19.8477 & 1.81 & -17.4789 & -18.8022 & -36.5609 \\
\hline $\mathrm{J} 1733-2228$ & 0.871683 & -16.3696 & 1.49 & -11.7858 & -17.5391 & -35.2133 \\
\hline $\mathrm{J} 1733-2533$ & 0.659794 & -14.6778 & 7.78 & -10.2149 & -15.7263 & -34.1184 \\
\hline J1733-2837 & 0.768185 & -14.9547 & 4.66 & -10.4258 & -16.0693 & -34.2387 \\
\hline $\mathrm{J} 1733-3030$ & 0.362052 & -14.7825 & 14.18 & -10.5803 & -15.5704 & -34.2232 \\
\hline $\mathrm{J} 1733-3322$ & 1.24592 & -14.3872 & 6.3 & -9.64827 & -15.7119 & -34.0123 \\
\hline J1733-3716 & 0.337586 & -13.8239 & 3.44 & -9.65207 & -14.5814 & -32.6191 \\
\hline J1733-4005 & 0.561778 & -14.4413 & 13.95 & -10.0483 & -15.42 & -34.0656 \\
\hline J1733-5515 & 1.01123 & -15.3979 & 4.11 & -10.7496 & -16.6319 & -34.7468 \\
\hline $\mathrm{J} 1734-0212$ & 0.839394 & -15.3757 & 5.36 & -10.8083 & -16.5288 & -34.7591 \\
\hline J1734-2415 & 0.612524 & -14.9788 & 3.48 & -10.5482 & -15.9951 & -34.0377 \\
\hline $\mathrm{J} 1734-3058$ & 0.541286 & -16.7905 & 4.37 & -12.4136 & -17.7531 & -35.8946 \\
\hline J1734-3333 & 1.16934 & -11.6421 & 7.4 & -6.93067 & -12.9392 & -31.3094 \\
\hline $\mathrm{J} 1735-0243$ & 0.782887 & -15.1993 & 3.25 & -10.6621 & -16.3221 & -34.3351 \\
\hline J1735-0724 & 0.419335 & -14.9172 & 4.32 & -10.6512 & -15.7689 & -33.9055 \\
\hline $\mathrm{J} 1735-3258$ & 0.350963 & -13.5834 & 11.14 & -9.39465 & -14.3578 & -32.9057 \\
\hline J1736-2457 & 2.64222 & -14.4647 & 4.34 & -9.39928 & -16.1158 & -34.2544 \\
\hline J1736-2819 & 1.59242 & -13.8268 & 4.86 & -8.9813 & -15.258 & -33.4457 \\
\hline J1736-2843 & 6.44504 & -13.5229 & 5.47 & -8.0702 & -15.5613 & -33.8003 \\
\hline J1736-3511 & 0.502803 & -14.8041 & 2.72 & -10.4592 & -15.7346 & -33.6703 \\
\hline $\mathrm{J} 1737-0811$ & 0.004175 & -20.1007 & 2.38 & -17.8366 & -18.9505 & -36.8282 \\
\hline $\mathrm{J} 1737-3102$ & 0.768672 & -13.4283 & 4.44 & -8.8991 & -14.5432 & -32.6916 \\
\hline J1737-3137 & 0.450432 & -12.857 & 5.88 & -8.5599 & -13.7398 & -32.0102 \\
\hline $\mathrm{J} 1737-3320$ & 0.816273 & -14.6478 & 14.08 & -10.0925 & -15.7888 & -34.4385 \\
\hline J1737-3555 & 0.397585 & -14.2132 & 2.25 & -9.97037 & -15.0418 & -32.8951 \\
\hline $\mathrm{J} 1738+0333$ & 0.00585 & -19.618 & 1.47 & -17.2074 & -18.6143 & -36.2827 \\
\hline $\mathrm{J} 1738-2330$ & 1.97885 & -14.0675 & 2.79 & -9.12766 & -15.5931 & -33.5398 \\
\hline $\mathrm{J} 1738-2647$ & 0.349591 & -14.5017 & 3.88 & -10.3147 & -15.2744 & -33.3643 \\
\hline
\end{tabular}


Table 1 continued

\begin{tabular}{|c|c|c|c|c|c|c|}
\hline Pulsar & $P(\mathrm{~s})$ & $\log \dot{P}$ & $d(\mathrm{kpc})$ & $\log \varepsilon$ & $\log \eta$ & $\log h$ \\
\hline $\mathrm{J} 1738-2736$ & 0.627716 & -14.3354 & 5.91 & -9.89414 & -15.3623 & -33.6349 \\
\hline J1738-2955 & 0.443398 & -13.0867 & 3.91 & -8.79647 & -13.9627 & -32.0559 \\
\hline J1738-3107 & 0.549498 & -15.5287 & 10.76 & -11.1453 & -16.4978 & -35.0307 \\
\hline $\mathrm{J} 1738-3211$ & 0.768499 & -15.0996 & 1.48 & -10.5705 & -16.2144 & -33.8857 \\
\hline $\mathrm{J} 1738-3316$ & 0.730373 & -16.0526 & 4.55 & -11.5456 & -17.1453 & -35.3043 \\
\hline $\mathrm{J} 1739+0612$ & 0.234169 & -15.8069 & 5.44 & -11.7939 & -16.4056 & -34.6422 \\
\hline $\mathrm{J} 1739-1313$ & 1.2157 & -16.0878 & 2.02 & -11.3595 & -17.4018 & -35.2082 \\
\hline J1739-2903 & 0.322882 & -14.1035 & 3.19 & -9.95098 & -14.8417 & -32.8465 \\
\hline $\mathrm{J} 1739-3023$ & 0.114368 & -13.9431 & 3.41 & -10.2413 & -14.2305 & -32.2644 \\
\hline J1739-3049 & 0.239317 & -14.6615 & 6.97 & -10.6391 & -15.2697 & -33.614 \\
\hline J1739-3131 & 0.529441 & -13.7305 & 7.68 & -9.36322 & -14.6835 & -33.0699 \\
\hline J1739-3159 & 0.877561 & -15.7055 & 4.85 & -11.1188 & -16.878 & -35.0648 \\
\hline J1739-3951 & 0.341772 & -16.7011 & 1.13 & -12.524 & -17.464 & -35.0182 \\
\hline $\mathrm{J} 1740+1000$ & 0.154087 & -13.6676 & 1.36 & -9.83634 & -14.0845 & -31.7191 \\
\hline $\mathrm{J} 1740+1311$ & 0.80305 & -14.8386 & 4.77 & -10.2904 & -15.9725 & -34.1521 \\
\hline $\mathrm{J} 1740-2540$ & 1.69266 & -14.7328 & 8.26 & -9.86081 & -16.1905 & -34.6086 \\
\hline $\mathrm{J} 1740-3015$ & 0.606887 & -12.3316 & 0.4 & -7.90505 & -13.3439 & -30.447 \\
\hline $\mathrm{J} 1740-3052$ & 0.570313 & -13.5935 & 10.84 & -9.19389 & -14.5787 & -33.1148 \\
\hline $\mathrm{J} 1740-3327$ & 0.515001 & -14.4089 & 4.72 & -10.0537 & -15.3499 & -33.5249 \\
\hline $\mathrm{J} 1741-0840$ & 2.04308 & -14.644 & 3.5 & -9.69024 & -16.1834 & -34.2285 \\
\hline $\mathrm{J} 1741+1351$ & 0.003747 & -19.52 & 1.08 & -17.3029 & -18.3228 & -35.8573 \\
\hline J1741-2019 & 3.90451 & -13.7878 & 2 & -8.55279 & -15.6085 & -33.4106 \\
\hline $\mathrm{J} 1741-2054$ & 0.4137 & -13.7696 & 0.3 & -9.50941 & -14.6154 & -31.5936 \\
\hline J1741-2719 & 0.346797 & -16.0937 & 5.74 & -11.9101 & -16.8629 & -35.1229 \\
\hline $\mathrm{J} 1741-2733$ & 0.892959 & -15.8297 & 3.33 & -11.2355 & -17.0097 & -35.0332 \\
\hline $\mathrm{J} 1741+2758$ & 1.36074 & -14.7352 & 2.06 & -9.95795 & -16.0981 & -33.913 \\
\hline J1741-2945 & 0.223558 & -15.1979 & 4.66 & -11.2051 & -15.7764 & -33.9459 \\
\hline $\mathrm{J} 1741-3016$ & 1.89375 & -14.0462 & 5.02 & -9.12546 & -15.5527 & -33.7545 \\
\hline J1741-3927 & 0.512211 & -14.7144 & 4.75 & -10.3615 & -15.653 & -33.8308 \\
\hline $\mathrm{J} 1742-3957$ & 1.01635 & -16.0883 & 6.4 & -11.4378 & -17.3245 & -35.6317 \\
\hline J1742-4616 & 0.412401 & -16.4711 & 4.99 & -12.2123 & -17.3156 & -35.5147 \\
\hline $\mathrm{J} 1743-0339$ & 0.444645 & -14.8069 & 1.59 & -10.5154 & -15.684 & -33.3865 \\
\hline $\mathrm{J} 1743-1351$ & 0.405337 & -15.3206 & 4.85 & -11.0693 & -16.1575 & -34.3443 \\
\hline $\mathrm{J} 1743-2442$ & 1.24251 & -15.3261 & 4.96 & -10.5883 & -16.6495 & -34.846 \\
\hline $\mathrm{J} 1743-3150$ & 2.41458 & -12.9172 & 3.65 & -7.89092 & -14.5292 & -32.5926 \\
\hline $\mathrm{J} 1743-3153$ & 0.193105 & -13.9747 & 8.03 & -10.0454 & -14.4896 & -32.8954 \\
\hline $\mathrm{J} 1743-4212$ & 0.306167 & -15.1062 & 4.67 & -10.9768 & -15.8213 & -33.9917 \\
\hline $\mathrm{J} 1744-1134$ & 0.004075 & -20.0491 & 0.4 & -17.7956 & -18.8884 & -35.9915 \\
\hline $\mathrm{J} 1744-1610$ & 1.75721 & -14.6234 & 1.99 & -9.73515 & -16.0974 & -33.8973 \\
\hline J1744-2335 & 1.68351 & -15.0794 & 2.45 & -10.2097 & -16.5347 & -34.4249 \\
\hline $\mathrm{J} 1744-3130$ & 1.06606 & -13.6737 & 3.64 & -9.00243 & -14.9306 & -32.9928 \\
\hline J1744-3922 & 0.172444 & -17.8097 & 4.6 & -13.9296 & -18.2755 & -36.4393 \\
\hline J1744-5337 & 0.355666 & -15.7212 & 8.16 & -11.5268 & -16.5014 & -34.9142 \\
\hline J1745-0129 & 1.04541 & -15.2 & 7.26 & -10.5372 & -16.4484 & -34.8104 \\
\hline $\mathrm{J} 1745-0952$ & 0.019376 & -19.0339 & 2.38 & -16.1031 & -18.5503 & -36.4279 \\
\hline $\mathrm{J} 1745+1017$ & 0.002652 & -20.5638 & 1.36 & -18.4968 & -19.2166 & -36.8512 \\
\hline J1745-2229 & 1.16059 & -14.5436 & 9.3 & -9.8355 & -15.8375 & -34.307 \\
\hline
\end{tabular}


Table 1 continued

\begin{tabular}{|c|c|c|c|c|c|c|}
\hline Pulsar & $P(\mathrm{~s})$ & $\log \dot{P}$ & $d(\mathrm{kpc})$ & $\log \varepsilon$ & $\log \eta$ & $\log h$ \\
\hline $\mathrm{J} 1745-2900$ & 3.76373 & -10.7545 & 8.3 & -5.53542 & -12.5593 & -30.9794 \\
\hline $\mathrm{J} 1745-3040$ & 0.367429 & -13.9706 & 0.2 & -9.76199 & -14.7649 & -31.567 \\
\hline $\mathrm{J} 1745-3812$ & 0.698353 & -14.6144 & 4.82 & -10.1269 & -15.6876 & -33.8717 \\
\hline $\mathrm{J} 1746+2245$ & 3.46504 & -14.308 & 4.32 & -9.12487 & -16.0769 & -34.2134 \\
\hline $\mathrm{J} 1746+2540$ & 1.05815 & -14.9788 & 4.16 & -10.3108 & -16.2325 & -34.3527 \\
\hline J1746-2849 & 1.47848 & -13.8962 & 30 & -9.08293 & -15.2952 & -34.2733 \\
\hline $\mathrm{J} 1746-2850$ & 1.0771 & -11.8729 & 12.86 & -7.19719 & -13.1343 & -31.7446 \\
\hline J1746-2856 & 0.945224 & -13.9031 & 21.77 & -9.2841 & -15.1078 & -33.9467 \\
\hline $\mathrm{J} 1747-1030$ & 1.57879 & -15.3665 & 7.27 & -10.5248 & -16.794 & -35.1566 \\
\hline $\mathrm{J} 1747-2647$ & 0.500254 & -13.8794 & 8.44 & -9.53678 & -14.8078 & -33.2352 \\
\hline $\mathrm{J} 1747-2802$ & 2.78008 & -14.6253 & 11.78 & -9.53774 & -16.2985 & -34.8707 \\
\hline J1747-2809 & 0.052153 & -12.8069 & 17.55 & -9.44614 & -12.7533 & -31.4986 \\
\hline $\mathrm{J} 1747-2958$ & 0.098814 & -13.2125 & 2.49 & -9.57427 & -13.4365 & -31.3338 \\
\hline J1747-4036 & 0.001646 & -19.8827 & 5.81 & -18.0228 & -18.3283 & -36.5935 \\
\hline J1748-1300 & 0.394133 & -14.9172 & 3.64 & -10.6781 & -15.742 & -33.8042 \\
\hline J1748-2444 & 0.442838 & -15.9547 & 3.89 & -11.665 & -16.8301 & -34.9211 \\
\hline J1749-2347 & 0.874486 & -14.6162 & 6.1 & -10.031 & -15.7871 & -34.0735 \\
\hline J1749-2629 & 1.33539 & -14.7645 & 5.28 & -9.99541 & -16.1192 & -34.3429 \\
\hline $\mathrm{J} 1749-3002$ & 0.609874 & -14.104 & 9.05 & -9.67533 & -15.1184 & -33.5761 \\
\hline $\mathrm{J} 1749-4931$ & 0.445822 & -15.2291 & 1.8 & -10.9365 & -16.1075 & -33.8638 \\
\hline J1749-5605 & 1.33231 & -14.6737 & 2.66 & -9.90561 & -16.0274 & -33.9534 \\
\hline J1750-2043 & 5.63905 & -14.0996 & 5.69 & -8.70497 & -16.08 & -34.3362 \\
\hline $\mathrm{J} 1750-2438$ & 0.712794 & -13.9666 & 7.15 & -9.47016 & -15.0487 & -33.4041 \\
\hline $\mathrm{J} 1750-2444$ & 0.899377 & -15.5784 & 5.03 & -10.981 & -16.7615 & -34.9641 \\
\hline J1750-28 & 1.30051 & -14.2381 & 5.19 & -9.48051 & -15.5813 & -33.7976 \\
\hline $\mathrm{J} 1750-3157$ & 0.910363 & -15.7055 & 4.43 & -11.1029 & -16.8939 & -35.0414 \\
\hline $\mathrm{J} 1750-3503$ & 0.684014 & -16.4191 & 5.07 & -11.9406 & -17.4833 & -35.6894 \\
\hline $\mathrm{J} 1751-2516$ & 0.394836 & -14.5784 & 7.46 & -10.3385 & -15.404 & -33.7778 \\
\hline J1751-2857 & 0.003915 & -19.9469 & 1.44 & -17.7107 & -18.7688 & -36.4282 \\
\hline $\mathrm{J} 1751-3323$ & 0.548227 & -14.054 & 9.27 & -9.67163 & -15.0221 & -33.4903 \\
\hline $\mathrm{J} 1751-4657$ & 0.742352 & -14.8894 & 1.03 & -10.3753 & -15.9892 & -33.5031 \\
\hline $\mathrm{J} 1752+2359$ & 0.409051 & -15.1918 & 2.7 & -10.9366 & -16.0327 & -33.9651 \\
\hline $\mathrm{J} 1752-2410$ & 0.191037 & -15.209 & 7.42 & -11.2844 & -15.7193 & -34.0907 \\
\hline J1752-2806 & 0.562558 & -14.0899 & 0.2 & -9.69629 & -15.0692 & -31.8713 \\
\hline J1752-2821 & 0.640229 & -14.4597 & 7.62 & -10.0099 & -15.4952 & -33.8782 \\
\hline J1753-1914 & 0.062955 & -17.6946 & 2.77 & -14.2522 & -17.7228 & -35.6664 \\
\hline $\mathrm{J} 1753-2240$ & 0.095138 & -18.0132 & 3.46 & -14.3914 & -18.2207 & -36.2609 \\
\hline $\mathrm{J} 1753-2501$ & 0.528336 & -13.8508 & 10.18 & -9.48442 & -14.8028 & -33.3116 \\
\hline $\mathrm{J} 1754-2422$ & 2.09025 & -15.0809 & 11.43 & -10.1173 & -16.6303 & -35.1894 \\
\hline $\mathrm{J} 1754-3443$ & 0.361691 & -15.2434 & 5.6 & -11.0416 & -16.0308 & -34.2801 \\
\hline $\mathrm{J} 1754-3510$ & 0.392704 & -15.1068 & 2.24 & -10.8693 & -15.93 & -33.7813 \\
\hline $\mathrm{J} 1754+5201$ & 2.3914 & -14.8069 & 3.56 & -9.78477 & -16.4147 & -34.4672 \\
\hline J1755-0903 & 0.19071 & -15.1073 & 2.16 & -11.1835 & -15.6169 & -33.4524 \\
\hline $\mathrm{J} 1755-1650$ & 0.733744 & -15.1637 & 4.27 & -10.6547 & -16.2584 & -34.3899 \\
\hline J1755-2025 & 0.322231 & -14.3546 & 8.64 & -10.203 & -15.0919 & -33.5295 \\
\hline $\mathrm{J} 1755-2521$ & 1.17597 & -13.0448 & 4.12 & -8.33094 & -14.3443 & -32.4603 \\
\hline $\mathrm{J} 1755-25211$ & 1.00451 & -13.5058 & 11.52 & -8.86044 & -14.7369 & -33.2995 \\
\hline
\end{tabular}


Table 1 continued

\begin{tabular}{|c|c|c|c|c|c|c|}
\hline Pulsar & $P(\mathrm{~s})$ & $\log \dot{P}$ & $d(\mathrm{kpc})$ & $\log \varepsilon$ & $\log \eta$ & $\log h$ \\
\hline $\mathrm{J} 1755-2534$ & 0.233541 & -13.9508 & 7.22 & -9.93897 & -14.5483 & -32.9079 \\
\hline J1755-26 & 0.430872 & -13.9101 & 5.17 & -9.63229 & -14.7736 & -32.9881 \\
\hline $\mathrm{J} 1755-2725$ & 0.261955 & -16.8633 & 2.83 & -12.8016 & -17.5107 & -35.4635 \\
\hline $\mathrm{J} 1755-3716$ & 0.012786 & -19.5072 & 6.38 & -16.7571 & -18.8431 & -37.149 \\
\hline $\mathrm{J} 1756-2225$ & 0.40498 & -13.2782 & 5.02 & -9.0273 & -14.1148 & -32.3165 \\
\hline J1756-2251 & 0.028462 & -17.9914 & 0.73 & -14.8937 & -17.6748 & -35.0392 \\
\hline $\mathrm{J} 1756-2435$ & 0.67048 & -15.5452 & 4.96 & -11.0753 & -16.6007 & -34.7972 \\
\hline J1756-2619 & 0.724514 & -14.9066 & 7.74 & -10.4031 & -15.9958 & -34.3856 \\
\hline $\mathrm{J} 1757-2223$ & 0.18531 & -15.1068 & 4.11 & -11.1954 & -15.6038 & -33.7187 \\
\hline J1757-2421 & 0.234101 & -13.8894 & 3.51 & -9.87655 & -14.488 & -32.5343 \\
\hline J1757-27 & 0.017687 & -18.6778 & 5.42 & -15.7867 & -18.1546 & -36.3896 \\
\hline $\mathrm{J} 1757-5322$ & 0.00887 & -19.58 & 1.36 & -16.9887 & -18.7571 & -36.3917 \\
\hline J1758-1931 & 0.692552 & -13.7721 & 4.23 & -9.28821 & -14.8417 & -32.9691 \\
\hline $\mathrm{J} 1758-2206$ & 0.430278 & -15.0195 & 11.38 & -10.7423 & -15.8824 & -34.4396 \\
\hline $\mathrm{J} 1758-2540$ & 2.10726 & -14.8097 & 3.85 & -9.8425 & -16.3625 & -34.4491 \\
\hline $\mathrm{J} 1758-2630$ & 1.20289 & -14.2865 & 4.99 & -9.56283 & -15.5959 & -33.795 \\
\hline $\mathrm{J} 1758-2846$ & 0.766706 & -16.0264 & 1.71 & -11.4983 & -17.1402 & -34.8742 \\
\hline $\mathrm{J} 1758+3030$ & 0.947256 & -15.1427 & 2.67 & -10.5227 & -16.3483 & -34.2759 \\
\hline J1759-1029 & 2.51226 & -13.8041 & 3.77 & -8.76058 & -15.4333 & -33.5107 \\
\hline J1759-1736 & 0.798452 & -15.5935 & 4.75 & -11.0478 & -16.7249 & -34.9026 \\
\hline J1759-1903 & 0.731505 & -14.5129 & 13.3 & -10.0052 & -15.6062 & -34.2311 \\
\hline J1759-1940 & 0.25472 & -16.0297 & 5.24 & -11.9801 & -16.6649 & -34.8853 \\
\hline J1759-1956 & 2.84339 & -13.7305 & 4.44 & -8.6332 & -15.4135 & -33.5619 \\
\hline J1759-2205 & 0.460974 & -13.9626 & 3.54 & -9.65544 & -14.8554 & -32.9055 \\
\hline $\mathrm{J} 1759-2302$ & 0.810718 & -13.9706 & 11.88 & -9.41829 & -15.1086 & -33.6845 \\
\hline $\mathrm{J} 1759-2307$ & 0.558889 & -14.4248 & 11.2 & -10.034 & -15.4013 & -33.9516 \\
\hline J1759-2549 & 0.956549 & -13.0017 & 5.94 & -8.37758 & -14.2116 & -32.4864 \\
\hline J1759-2922 & 0.5744 & -14.3344 & 1.92 & -9.93175 & -15.3228 & -33.1071 \\
\hline J1759-3107 & 1.07895 & -14.4237 & 3.36 & -9.7472 & -15.6858 & -33.7132 \\
\hline $\mathrm{J} 1800-0125$ & 0.783185 & -13.9393 & 2.24 & -9.40199 & -15.0623 & -32.9136 \\
\hline $\mathrm{J} 1800-2114$ & 1.79927 & -15.2596 & 11.18 & -10.3611 & -16.7439 & -35.2934 \\
\hline $\mathrm{J} 1801-0357$ & 0.921491 & -14.4802 & 7.13 & -9.87223 & -15.6738 & -34.028 \\
\hline $\mathrm{J} 1801-1417$ & 0.003625 & -20.2749 & 1.8 & -18.0721 & -19.0634 & -36.8197 \\
\hline $\mathrm{J} 1801-1855$ & 2.5505 & -15.7399 & 11.91 & -10.6899 & -17.3757 & -35.9527 \\
\hline J1801-1909 & 1.10872 & -15.153 & 4.81 & -10.4648 & -16.427 & -34.6102 \\
\hline $\mathrm{J} 1801-2115$ & 0.438113 & -16.8097 & 12.26 & -12.5246 & -17.6804 & -36.27 \\
\hline $\mathrm{J} 1801-2154$ & 0.375297 & -13.7959 & 5.16 & -9.57805 & -14.5994 & -32.8131 \\
\hline J1801-2304 & 0.415827 & -12.9469 & 4 & -8.68456 & -13.795 & -31.8981 \\
\hline $\mathrm{J} 1801-2451$ & 0.124924 & -12.8928 & 4.61 & -9.15269 & -13.2186 & -31.3833 \\
\hline J1801-2920 & 1.08191 & -14.4828 & 3.27 & -9.80516 & -15.7461 & -33.7617 \\
\hline $\mathrm{J} 1801-3458$ & 1.3856 & -15.4012 & 4.96 & -10.6161 & -16.772 & -34.9685 \\
\hline $\mathrm{J} 1802+0128$ & 0.554262 & -14.6757 & 8.79 & -10.2885 & -15.6486 & -34.0936 \\
\hline $\mathrm{J} 1802-1745$ & 0.514671 & -15.2487 & 5.22 & -10.8937 & -16.1894 & -34.4081 \\
\hline $\mathrm{J} 1802-2124$ & 0.012648 & -19.1391 & 3.33 & -16.3936 & -18.4702 & -36.4937 \\
\hline $\mathrm{J} 1802-2426$ & 0.569007 & -14.0675 & 12.13 & -9.66896 & -15.0518 & -33.6367 \\
\hline $\mathrm{J} 1802-3346$ & 2.46105 & -14.8794 & 10.24 & -9.84485 & -16.4997 & -35.0111 \\
\hline J1803-1616 & 0.536596 & -14.752 & 12.9 & -10.3789 & -15.7108 & -34.3225 \\
\hline
\end{tabular}


Table 1 continued

\begin{tabular}{|c|c|c|c|c|c|c|}
\hline Pulsar & $P(\mathrm{~s})$ & $\log \dot{P}$ & $d(\mathrm{kpc})$ & $\log \varepsilon$ & $\log \eta$ & $\log h$ \\
\hline J1803-1857 & 2.86434 & -13.8182 & 5.88 & -8.71768 & -15.5043 & -33.7748 \\
\hline J1803-1920 & 0.443649 & -15.4815 & 6.46 & -11.191 & -16.3577 & -34.669 \\
\hline J1803-2137 & 0.133667 & -12.8729 & 4.4 & -9.10342 & -13.2281 & -31.3726 \\
\hline $\mathrm{J} 1803-2712$ & 0.334415 & -16.767 & 3.62 & -12.5993 & -17.5204 & -35.5802 \\
\hline J1803-3329 & 0.633412 & -15.4724 & 6.01 & -11.0272 & -16.5032 & -34.7831 \\
\hline J1804-0735 & 0.023101 & -18.3307 & 7.8 & -15.3236 & -17.9235 & -36.3166 \\
\hline $\mathrm{J} 1804-2228$ & 0.570511 & -15.8447 & 5.33 & -11.4449 & -16.8301 & -35.0579 \\
\hline J1804-2717 & 0.009343 & -19.3883 & 1.17 & -16.7743 & -18.5879 & -36.1572 \\
\hline $\mathrm{J} 1805+0306$ & 0.218712 & -15.0004 & 5 & -11.0171 & -15.5695 & -33.7695 \\
\hline J1805-0619 & 0.454651 & -15.0137 & 6.69 & -10.7125 & -15.9005 & -34.227 \\
\hline J1805-1504 & 1.18127 & -15.5638 & 5.3 & -10.848 & -16.8653 & -35.0907 \\
\hline $\mathrm{J} 1805-2032$ & 0.405769 & -14.0762 & 11.87 & -9.82451 & -14.9137 & -33.4892 \\
\hline J1805-2037 & 0.357807 & -14.7545 & 9.26 & -10.5574 & -15.5373 & -34.005 \\
\hline $\mathrm{J} 1805-2447$ & 0.661402 & -17.2351 & 4.79 & -12.7712 & -18.2847 & -36.4661 \\
\hline J1805-2948 & 0.428341 & -15.3242 & 4.61 & -11.049 & -16.1852 & -34.3499 \\
\hline $\mathrm{J} 1806+1023$ & 0.484286 & -16.2418 & 3.27 & -11.9133 & -17.1561 & -35.1717 \\
\hline J1806-1154 & 0.522618 & -14.8508 & 3.56 & -10.4891 & -15.7981 & -33.8506 \\
\hline J1806-1618 & 0.668309 & -15.0645 & 6.36 & -10.5961 & -16.1186 & -34.4231 \\
\hline J1806-1920 & 0.879791 & -16.7696 & 10.16 & -12.1817 & -17.9431 & -36.451 \\
\hline J1806-2125 & 0.481789 & -12.9172 & 10.02 & -8.59091 & -13.8292 & -32.3311 \\
\hline $\mathrm{J} 1807+0756$ & 0.4643 & -15.8894 & 7.66 & -11.5792 & -16.7854 & -35.1706 \\
\hline J1807-0847 & 0.163727 & -16.5406 & 1.5 & -12.683 & -16.9839 & -34.661 \\
\hline $\mathrm{J} 1807-2557$ & 2.76419 & -14.3019 & 11.21 & -9.21688 & -15.9726 & -34.5233 \\
\hline J1807-2715 & 0.827776 & -13.9136 & 9.63 & -9.35227 & -15.0607 & -33.5454 \\
\hline J1808-0813 & 0.876044 & -14.9066 & 5.18 & -10.3206 & -16.0783 & -34.2936 \\
\hline $\mathrm{J} 1808-1020$ & 0.596993 & -15.1124 & 8.5 & -10.693 & -16.1175 & -34.548 \\
\hline $\mathrm{J} 1808-1517$ & 0.544549 & -14.5735 & 4.16 & -10.194 & -15.5387 & -33.6588 \\
\hline $\mathrm{J} 1808-1726$ & 0.241035 & -16.9355 & 8.57 & -12.91 & -17.5468 & -35.9808 \\
\hline J1808-2024 & 7.55592 & -9.26043 & 13 & -3.73869 & -11.3679 & -29.9829 \\
\hline J1808-2057 & 0.91841 & -13.767 & 7.35 & -9.16051 & -14.9592 & -33.3265 \\
\hline J1808-2701 & 2.45788 & -13.1818 & 2.45 & -8.14776 & -14.8015 & -32.6917 \\
\hline J1808-3249 & 0.364912 & -14.1518 & 5.06 & -9.94617 & -14.9431 & -33.1484 \\
\hline J1809-0119 & 0.744976 & -14.6402 & 10.59 & -10.1246 & -15.7415 & -34.2674 \\
\hline J1809-0743 & 0.313886 & -15.8182 & 17.82 & -11.6779 & -16.5441 & -35.296 \\
\hline J1809-1429 & 0.895285 & -14.2807 & 12.01 & -9.68525 & -15.4618 & -34.0424 \\
\hline $\mathrm{J} 1809-1850$ & 1.12448 & -13.9747 & 7.06 & -9.28029 & -15.2548 & -33.6047 \\
\hline J1809-1917 & 0.082747 & -13.5935 & 3.71 & -10.0323 & -13.7404 & -31.8108 \\
\hline J1809-1943 & 5.54035 & -11.1096 & 3.6 & -5.72259 & -13.0823 & -31.1396 \\
\hline J1809-2004 & 0.434811 & -14.1379 & 10.91 & -9.85612 & -15.0053 & -33.5442 \\
\hline J1809-2109 & 0.702414 & -14.4179 & 5.2 & -9.92789 & -15.4937 & -33.7107 \\
\hline $\mathrm{J} 1809-3547$ & 0.860388 & -15.9208 & 13.02 & -11.3427 & -17.0847 & -35.7003 \\
\hline $\mathrm{J} 1810-1441$ & 0.217214 & -16.6216 & 5.88 & -12.6413 & -17.1876 & -35.4581 \\
\hline $\mathrm{J} 1810-1820$ & 0.153716 & -16.2823 & 5.59 & -12.4522 & -16.6982 & -34.9467 \\
\hline J1810-2005 & 0.032822 & -18.8327 & 4.05 & -15.6731 & -18.578 & -36.6865 \\
\hline J1810-5338 & 0.261049 & -15.4168 & 2 & -11.3566 & -16.0627 & -33.8648 \\
\hline $\mathrm{J} 1811-0154$ & 0.924945 & -14.7932 & 9.56 & -10.1836 & -15.9884 & -34.47 \\
\hline $\mathrm{J} 1811+0702$ & 0.461713 & -14.5702 & 3.13 & -10.2624 & -15.4638 & -33.4604 \\
\hline
\end{tabular}


Table 1 continued

\begin{tabular}{|c|c|c|c|c|c|c|}
\hline Pulsar & $P(\mathrm{~s})$ & $\log \dot{P}$ & $d(\mathrm{kpc})$ & $\log \varepsilon$ & $\log \eta$ & $\log h$ \\
\hline J1811-1049 & 2.62386 & -15.0969 & 8.5 & -10.0345 & -16.745 & -35.1755 \\
\hline J1811-1717 & 0.391385 & -15.9208 & 7.03 & -11.6848 & -16.7426 & -35.0906 \\
\hline J1811-1736 & 0.104182 & -18.0453 & 5.93 & -14.384 & -18.2922 & -36.5663 \\
\hline $\mathrm{J} 1811-1835$ & 0.557464 & -14.2 & 9.36 & -9.8103 & -15.1753 & -33.6477 \\
\hline J1811-1925 & 0.064667 & -13.3565 & 5 & -9.90241 & -13.3964 & -31.5964 \\
\hline $\mathrm{J} 1811-2405$ & 0.002661 & -19.8729 & 1.7 & -17.8044 & -18.5271 & -36.2586 \\
\hline $\mathrm{J} 1811-2439$ & 0.415813 & -15.5272 & 3.85 & -11.2649 & -16.3753 & -34.4618 \\
\hline J1811-4930 & 1.4327 & -14.6478 & 2.01 & -9.84821 & -16.0331 & -33.8374 \\
\hline $\mathrm{J} 1812+0226$ & 0.793903 & -14.4437 & 6.16 & -9.90048 & -15.5726 & -33.8633 \\
\hline $\mathrm{J} 1812-1718$ & 1.20537 & -13.719 & 4.2 & -8.99439 & -15.0292 & -33.1535 \\
\hline J1812-1733 & 0.538341 & -15.0079 & 6.32 & -10.6334 & -15.9681 & -34.2699 \\
\hline $\mathrm{J} 1812-1910$ & 0.430991 & -13.4237 & 11.54 & -9.14574 & -14.2873 & -32.8505 \\
\hline $\mathrm{J} 1812-2102$ & 1.22335 & -13.6216 & 9.62 & -8.8906 & -14.9383 & -33.4225 \\
\hline $\mathrm{J} 1812-2526$ & 0.315835 & -15.752 & 14.71 & -11.6091 & -16.4806 & -35.1493 \\
\hline $\mathrm{J} 1812-2748$ & 0.236983 & -15.5003 & 3.03 & -11.4821 & -16.1042 & -34.0867 \\
\hline J1812-3039 & 0.587477 & -15.1805 & 4.61 & -10.768 & -16.1786 & -34.3434 \\
\hline J1813-1749 & 0.044699 & -12.8962 & 4.7 & -9.60245 & -12.7756 & -30.9488 \\
\hline $\mathrm{J} 1813+1822$ & 0.336425 & -16.6778 & 6.24 & -12.5074 & -17.4338 & -35.7301 \\
\hline J1813-2113 & 0.426466 & -14.6819 & 8.75 & -10.4086 & -15.541 & -33.984 \\
\hline J1813-2242 & 0.328514 & -16.3206 & 6.78 & -12.1606 & -17.0663 & -35.3986 \\
\hline $\mathrm{J} 1813-2621$ & 0.00443 & -19.9031 & 3.37 & -17.6132 & -18.7786 & -36.8073 \\
\hline $\mathrm{J} 1813+4013$ & 0.931089 & -14.5935 & 4.32 & -9.98102 & -15.7916 & -33.9281 \\
\hline $\mathrm{J} 1814-0521$ & 1.01422 & -15.0535 & 4.37 & -10.404 & -16.2888 & -34.4304 \\
\hline $\mathrm{J} 1814-0618$ & 1.37787 & -15.5346 & 5.63 & -10.752 & -16.903 & -35.1545 \\
\hline $\mathrm{J} 1814+1130$ & 0.751261 & -14.7799 & 4.53 & -10.2606 & -15.8848 & -34.042 \\
\hline J1814-1649 & 0.957464 & -14.1986 & 9.62 & -9.57402 & -15.4089 & -33.8931 \\
\hline J1814-1744 & 3.97591 & -12.1278 & 9.77 & -6.88495 & -13.9564 & -32.4474 \\
\hline $\mathrm{J} 1815-1738$ & 0.198436 & -13.1085 & 9.01 & -9.16739 & -13.6352 & -32.091 \\
\hline J1815-1910 & 1.24992 & -13.4401 & 7.69 & -8.69976 & -14.7661 & -33.1531 \\
\hline J1816-0755 & 0.217643 & -14.1884 & 3.21 & -10.2072 & -14.7553 & -32.7629 \\
\hline J1816-1446 & 0.5945 & -14.8761 & 8.98 & -10.4585 & -15.8794 & -34.3338 \\
\hline J1816-1729 & 0.782313 & -14.1391 & 6.48 & -9.60223 & -15.2616 & -33.5742 \\
\hline J1816-2650 & 0.592885 & -16.1778 & 3.61 & -11.7614 & -17.18 & -35.2385 \\
\hline $\mathrm{J} 1816+4510$ & 0.003193 & -19.3655 & 4.2 & -17.2179 & -18.0989 & -36.2232 \\
\hline J1816-5643 & 0.217923 & -17.7144 & 3.06 & -13.7327 & -18.2819 & -36.2687 \\
\hline $\mathrm{J} 1817-1511$ & 0.224604 & -14.8447 & 11.58 & -10.8498 & -15.4252 & -33.99 \\
\hline J1817-1938 & 2.04684 & -15.4437 & 10.74 & -10.4892 & -16.9839 & -35.516 \\
\hline $\mathrm{J} 1817-3618$ & 0.387017 & -14.6882 & 3.78 & -10.4571 & -15.5051 & -33.5837 \\
\hline J1817-3837 & 0.384487 & -15.2366 & 5.23 & -11.0082 & -16.0506 & -34.2702 \\
\hline J1818-1116 & 0.5448 & -14.4179 & 10.29 & -10.0382 & -15.3833 & -33.8968 \\
\hline $\mathrm{J} 1818-1422$ & 0.291489 & -14.6904 & 8.1 & -10.5823 & -15.3841 & -33.7937 \\
\hline J1818-1448 & 0.281371 & -14.2111 & 7.96 & -10.1184 & -14.8896 & -33.2915 \\
\hline $\mathrm{J} 1818-1519$ & 0.93969 & -14.3862 & 9.98 & -9.76972 & -15.5883 & -34.0885 \\
\hline $\mathrm{J} 1818-1541$ & 0.551134 & -14.0141 & 8.24 & -9.62941 & -14.9845 & -33.4015 \\
\hline $\mathrm{J} 1818-1556$ & 0.952709 & -15.1512 & 4.01 & -10.5288 & -16.3593 & -34.4635 \\
\hline $\mathrm{J} 1819-0925$ & 0.852047 & -14.5045 & 11.1 & -9.93054 & -15.6641 & -34.2104 \\
\hline J1819-1008 & 0.30149 & -14.8794 & 10.75 & -10.7567 & -15.5878 & -34.1203 \\
\hline
\end{tabular}


Table 1 continued

\begin{tabular}{|c|c|c|c|c|c|c|}
\hline Pulsar & $P(\mathrm{~s})$ & $\log \dot{P}$ & $d(\mathrm{kpc})$ & $\log \varepsilon$ & $\log \eta$ & $\log h$ \\
\hline $\mathrm{J} 1819-1114$ & 0.294163 & -15.2472 & 5.82 & -11.1351 & -15.9449 & -34.2109 \\
\hline $\mathrm{J} 1819-1131$ & 1.38814 & -15.1186 & 12.25 & -10.3327 & -16.4902 & -35.0794 \\
\hline $\mathrm{J} 1819+1305$ & 1.06036 & -15.4449 & 4.41 & -10.776 & -16.6995 & -34.845 \\
\hline J1819-1318 & 1.5157 & -15.224 & 1.52 & -10.4 & -16.6338 & -34.3167 \\
\hline $\mathrm{J} 1819-1408$ & 1.78849 & -14.5867 & 12.45 & -9.69076 & -16.0683 & -34.6646 \\
\hline $\mathrm{J} 1819-1458$ & 4.26316 & -12.2403 & 3.81 & -6.96715 & -14.0992 & -32.1812 \\
\hline $\mathrm{J} 1819-1510$ & 0.226539 & -17.1051 & 5.61 & -13.1065 & -17.6894 & -35.9394 \\
\hline J1819-1717 & 0.393522 & -14.4622 & 5.87 & -10.2238 & -15.2863 & -33.556 \\
\hline J1820-0427 & 0.598076 & -14.1986 & 0.3 & -9.77839 & -15.2045 & -32.1827 \\
\hline J1820-0509 & 0.337321 & -15.0306 & 2.81 & -10.8591 & -15.7878 & -33.7375 \\
\hline $\mathrm{J} 1820-1346$ & 0.92146 & -14.3468 & 9.63 & -9.73886 & -15.5404 & -34.0251 \\
\hline J1820-1529 & 0.333243 & -13.4214 & 9.6 & -9.25515 & -14.1733 & -32.6566 \\
\hline J1820-1818 & 0.309905 & -16.0287 & 8.43 & -11.894 & -16.7491 & -35.176 \\
\hline $\mathrm{J} 1821+0155$ & 0.033781 & -19.5376 & 2.29 & -16.3655 & -19.2954 & -37.1563 \\
\hline $\mathrm{J} 1821-0256$ & 0.414111 & -16.4295 & 2.55 & -12.1689 & -17.2757 & -35.1833 \\
\hline $\mathrm{J} 1821-0331$ & 0.902316 & -14.5969 & 5.56 & -9.99807 & -15.7814 & -34.0275 \\
\hline $\mathrm{J} 1821-1419$ & 1.65601 & -12.0482 & 11.93 & -7.18566 & -13.4964 & -32.074 \\
\hline $\mathrm{J} 1821-1432$ & 1.91513 & -14.27 & 6.95 & -9.34437 & -15.7814 & -34.1244 \\
\hline $\mathrm{J} 1821+1715$ & 1.36668 & -15.06 & 4.68 & -10.2809 & -16.4248 & -34.5961 \\
\hline $\mathrm{J} 1822+0705$ & 1.36282 & -14.757 & 3.02 & -9.97907 & -16.1205 & -34.1016 \\
\hline $\mathrm{J} 1822-0848$ & 2.50452 & -15.3947 & 4.19 & -10.3525 & -17.0226 & -35.1458 \\
\hline J1822-0907 & 0.9747 & -15.4498 & 12.31 & -10.8174 & -16.6678 & -35.2591 \\
\hline $\mathrm{J} 1822+1120$ & 1.78704 & -14.6289 & 8.77 & -9.73335 & -16.1102 & -34.5543 \\
\hline $\mathrm{J} 1822-1252$ & 2.07104 & -13.0716 & 10.61 & -8.11196 & -14.6169 & -33.1437 \\
\hline $\mathrm{J} 1822-1400$ & 0.214771 & -15.0424 & 7.77 & -11.067 & -15.6035 & -33.995 \\
\hline J1822-1617 & 0.831156 & -14.7235 & 11.64 & -10.1604 & -15.8724 & -34.4394 \\
\hline $\mathrm{J} 1822-2256$ & 1.87427 & -14.8697 & 3.43 & -9.95338 & -16.3716 & -34.408 \\
\hline J1822-4209 & 0.456512 & -15.3382 & 3.5 & -11.0353 & -16.2268 & -34.2719 \\
\hline $\mathrm{J} 1823-0154$ & 0.759777 & -14.9469 & 4.56 & -10.4228 & -16.0568 & -34.2168 \\
\hline $\mathrm{J} 1823+0550$ & 0.752907 & -15.644 & 2 & -11.1238 & -16.7499 & -34.552 \\
\hline $\mathrm{J} 1823-1115$ & 0.279829 & -14.8601 & 6.28 & -10.7698 & -15.5362 & -33.8352 \\
\hline $\mathrm{J} 1823-1126$ & 1.84653 & -13.4377 & 8.72 & -8.5279 & -14.9332 & -33.3748 \\
\hline J1823-1347 & 0.617107 & -14.0177 & 11.14 & -9.58392 & -15.0372 & -33.5852 \\
\hline J1823-1526 & 1.6254 & -14.3449 & 9.17 & -9.49045 & -15.785 & -34.2484 \\
\hline J1823-1807 & 1.63679 & -15.5544 & 6.64 & -10.697 & -16.9975 & -35.3208 \\
\hline J1823-3106 & 0.284054 & -14.5331 & 1.66 & -10.4363 & -15.2157 & -32.9368 \\
\hline $\mathrm{J} 1824-0127$ & 2.49947 & -14.4078 & 2.21 & -9.36652 & -16.0348 & -33.8803 \\
\hline $\mathrm{J} 1824-1118$ & 0.435759 & -14.4498 & 8.03 & -10.1671 & -15.3182 & -33.7239 \\
\hline J1824-1159 & 0.362492 & -14.2692 & 6.09 & -10.0665 & -15.0577 & -33.3433 \\
\hline $\mathrm{J} 1824-1350$ & 1.3966 & -15.2168 & 7.06 & -10.4283 & -16.591 & -34.9409 \\
\hline J1824-1423 & 0.359394 & -15.4067 & 6.05 & -11.2077 & -16.1914 & -34.4742 \\
\hline $\mathrm{J} 1824-1500$ & 0.41223 & -15.1203 & 8.3 & -10.8617 & -15.9646 & -34.3848 \\
\hline J1824-1945 & 0.189335 & -14.2815 & 3.7 & -10.3608 & -14.7879 & -32.8571 \\
\hline J1824-2233 & 1.16174 & -15.5214 & 4.26 & -10.8129 & -16.8157 & -34.9462 \\
\hline J1824-2328 & 1.50588 & -14.7595 & 5.76 & -9.93821 & -16.1664 & -34.4279 \\
\hline J1824-2537 & 0.22332 & -15.3565 & 5.48 & -11.3642 & -15.9346 & -34.1745 \\
\hline $\mathrm{J} 1825+0004$ & 0.778949 & -15.0575 & 2.26 & -10.5225 & -16.1782 & -34.0333 \\
\hline
\end{tabular}


Table 1 continued

\begin{tabular}{|c|c|c|c|c|c|c|}
\hline Pulsar & $P(\mathrm{~s})$ & $\log \dot{P}$ & $d(\mathrm{kpc})$ & $\log \varepsilon$ & $\log \eta$ & $\log h$ \\
\hline J1825-0319 & 0.004554 & -20.1675 & 3.26 & -17.8656 & -19.055 & -37.0693 \\
\hline $\mathrm{J} 1825-0935$ & 0.769006 & -13.2798 & 0.3 & -8.75046 & -14.3949 & -31.3731 \\
\hline $\mathrm{J} 1825-1108$ & 1.92587 & -14.7212 & 2.71 & -9.79317 & -16.235 & -34.169 \\
\hline J1825-1446 & 0.279187 & -13.644 & 5.45 & -9.55463 & -14.319 & -32.5565 \\
\hline J1826-1131 & 2.09314 & -14.3089 & 4.83 & -9.34467 & -15.8589 & -34.0439 \\
\hline J1826-1334 & 0.101487 & -13.1232 & 4.12 & -9.47334 & -13.3588 & -31.4747 \\
\hline J1826-1419 & 0.77062 & -14.0565 & 3.59 & -9.52621 & -15.1725 & -33.2286 \\
\hline J1826-1526 & 0.382073 & -14.9666 & 10.87 & -10.741 & -15.7779 & -34.3152 \\
\hline $\mathrm{J} 1827-0750$ & 0.270502 & -14.8125 & 7.39 & -10.7369 & -15.4738 & -33.8435 \\
\hline J1827-0934 & 0.512548 & -14.1409 & 4.47 & -9.78767 & -15.0797 & -33.2311 \\
\hline $\mathrm{J} 1827-0958$ & 0.245757 & -15 & 6.15 & -10.966 & -15.6197 & -33.9096 \\
\hline $\mathrm{J} 1828-0611$ & 0.269415 & -14.8356 & 8.76 & -10.7618 & -15.4952 & -33.9388 \\
\hline J1828-1007 & 0.153197 & -15.2125 & 4.72 & -11.3838 & -15.6269 & -33.8019 \\
\hline $\mathrm{J} 1828-1057$ & 0.246328 & -13.684 & 4.27 & -9.64906 & -14.3047 & -32.4362 \\
\hline J1828-1101 & 0.072052 & -13.8297 & 7.26 & -10.3286 & -13.9165 & -32.2785 \\
\hline J1828-1336 & 0.860332 & -15.0022 & 7.99 & -10.4241 & -16.166 & -34.5696 \\
\hline $\mathrm{J} 1828+1359$ & 0.74164 & -15.1373 & 3.29 & -10.6236 & -16.2366 & -34.2549 \\
\hline J1828-2119 & 0.514523 & -14.8962 & 12.56 & -10.5413 & -15.8367 & -34.4368 \\
\hline $\mathrm{J} 1829+0000$ & 0.199147 & -15.2798 & 3.32 & -11.3372 & -15.8082 & -33.8304 \\
\hline J1829-0734 & 0.318401 & -14.3197 & 5.45 & -10.1732 & -15.0518 & -33.2892 \\
\hline J1829-1751 & 0.307133 & -14.2557 & 5.49 & -10.1249 & -14.9722 & -33.2128 \\
\hline $\mathrm{J} 1829+2456$ & 0.04101 & -19.2798 & 0.74 & -16.0235 & -19.1219 & -36.4922 \\
\hline $\mathrm{J} 1830-0052$ & 0.345698 & -15.6253 & 7.1 & -11.4431 & -16.3931 & -34.7454 \\
\hline $\mathrm{J} 1830-0131$ & 0.457536 & -14.1993 & 2.68 & -9.8954 & -15.0889 & -33.018 \\
\hline J1830-1059 & 0.405043 & -13.2218 & 3.58 & -8.97089 & -14.0585 & -32.1134 \\
\hline $\mathrm{J} 1830-1135$ & 6.22155 & -13.3215 & 4.36 & -7.88413 & -15.3445 & -33.4851 \\
\hline $\mathrm{J} 1830-1313$ & 0.747188 & -14.767 & 10.42 & -10.2501 & -15.8696 & -34.3885 \\
\hline J1830-1414 & 0.771493 & -16.118 & 7.81 & -11.5873 & -17.2345 & -35.6282 \\
\hline $\mathrm{J} 1831-0823$ & 0.612133 & -15.51 & 4.41 & -11.0797 & -16.526 & -34.6715 \\
\hline $\mathrm{J} 1831-0952$ & 0.067267 & -14.0799 & 4.33 & -10.6086 & -14.1368 & -32.2744 \\
\hline $\mathrm{J} 1831-1223$ & 2.85794 & -14.262 & 5.51 & -9.16251 & -15.9472 & -34.1894 \\
\hline J1831-1329 & 2.16568 & -14.5243 & 6.48 & -9.54528 & -16.0891 & -34.4017 \\
\hline $\mathrm{J} 1831-1423$ & 0.507945 & -14.9626 & 8.02 & -10.6133 & -15.8975 & -34.3028 \\
\hline $\mathrm{J} 1832+0029$ & 0.533917 & -14.821 & 1.45 & -10.4501 & -15.7776 & -33.4401 \\
\hline $\mathrm{J} 1832-0644$ & 0.744295 & -13.4306 & 8.81 & -8.91543 & -14.5315 & -32.9776 \\
\hline J1832-0827 & 0.647293 & -13.1945 & 5.2 & -8.73995 & -14.2347 & -32.4518 \\
\hline J1832-0836 & 0.002719 & -20.0645 & 1.4 & -17.9866 & -18.728 & -36.3752 \\
\hline J1832-1021 & 0.330354 & -14.3768 & 6.43 & -10.2143 & -15.1249 & -33.4341 \\
\hline J1833-0209 & 0.291931 & -14.5607 & 9.25 & -10.4519 & -15.2551 & -33.7223 \\
\hline $\mathrm{J} 1833-0338$ & 0.686704 & -13.3809 & 5.07 & -8.90068 & -14.4468 & -32.6529 \\
\hline $\mathrm{J} 1833-0556$ & 1.52154 & -14.8928 & 7.37 & -10.0671 & -16.3042 & -34.6727 \\
\hline J1833-0559 & 0.483459 & -13.9101 & 5.83 & -9.58228 & -14.8236 & -33.0903 \\
\hline J1833-0827 & 0.085284 & -14.0376 & 4.5 & -10.4633 & -14.1976 & -32.3519 \\
\hline $\mathrm{J} 1833-1034$ & 0.061884 & -12.6946 & 4.1 & -9.25962 & -12.7154 & -30.8292 \\
\hline $\mathrm{J} 1833-1055$ & 0.63364 & -15.2782 & 8.44 & -10.8329 & -16.3092 & -34.7366 \\
\hline $\mathrm{J} 1833-6023$ & 1.88944 & -15.5686 & 1.85 & -10.6489 & -17.0741 & -34.8423 \\
\hline $\mathrm{J} 1834-0010$ & 0.520954 & -16.9788 & 2.64 & -12.6186 & -17.9248 & -35.8474 \\
\hline
\end{tabular}


Table 1 continued

\begin{tabular}{|c|c|c|c|c|c|c|}
\hline Pulsar & $P(\mathrm{~s})$ & $\log \dot{P}$ & $d(\mathrm{kpc})$ & $\log \varepsilon$ & $\log \eta$ & $\log h$ \\
\hline $\mathrm{J} 1834-0031$ & 0.329532 & -15.3478 & 4.35 & -11.1864 & -16.0948 & -34.2343 \\
\hline $\mathrm{J} 1834-0426$ & 0.290108 & -16.1433 & 2.3 & -12.0373 & -16.835 & -34.6978 \\
\hline $\mathrm{J} 1834-0602$ & 0.487914 & -14.7375 & 6.6 & -10.4058 & -15.655 & -33.9756 \\
\hline $\mathrm{J} 1834-0633$ & 0.317315 & -15.219 & 9.36 & -11.074 & -15.9496 & -34.4219 \\
\hline J1834-0731 & 0.51298 & -13.2351 & 4.76 & -8.88152 & -14.1743 & -32.353 \\
\hline $\mathrm{J} 1834-0742$ & 0.788354 & -13.4881 & 6.91 & -8.94794 & -14.614 & -32.9545 \\
\hline $\mathrm{J} 1834-0812$ & 0.491142 & -14.0017 & 10.34 & -9.66708 & -14.9221 & -33.4377 \\
\hline J1834-1202 & 0.610259 & -17.1713 & 6.28 & -12.7424 & -18.186 & -36.485 \\
\hline $\mathrm{J} 1834-1710$ & 0.358306 & -16.3288 & 3.53 & -12.1311 & -17.1122 & -35.1611 \\
\hline J1834-1855 & 1.46566 & -14.7144 & 6.21 & -9.90496 & -16.1096 & -34.4038 \\
\hline $\mathrm{J} 1835-0114$ & 0.005116 & -20.1549 & 2.67 & -17.8025 & -19.093 & -37.0206 \\
\hline J1835-0349 & 0.841865 & -14.5143 & 5.25 & -9.94558 & -15.6687 & -33.8899 \\
\hline $\mathrm{J} 1835-0522$ & 1.08775 & -15.3251 & 7.13 & -10.6452 & -16.5908 & -34.945 \\
\hline J1835-0643 & 0.30583 & -13.3925 & 6.44 & -9.26361 & -14.1072 & -32.4171 \\
\hline J1835-0924 & 0.859192 & -13.6696 & 6.62 & -9.09204 & -14.8328 & -33.1547 \\
\hline J1835-09242 & 0.235249 & -16.9281 & 6.85 & -12.9131 & -17.5288 & -35.8655 \\
\hline J1835-0928 & 0.621734 & -15.0137 & 6.36 & -10.5766 & -16.0364 & -34.3409 \\
\hline J1835-0944 & 0.145347 & -14.3575 & 4.67 & -10.5517 & -14.7491 & -32.9195 \\
\hline J1835-0946 & 0.379536 & -16.3645 & 4.04 & -12.1418 & -17.1729 & -35.2804 \\
\hline $\mathrm{J} 1835-1020$ & 0.302448 & -14.2277 & 2.57 & -10.1036 & -14.9375 & -32.8485 \\
\hline J1835-1106 & 0.165907 & -13.6861 & 3.08 & -9.82282 & -14.1351 & -32.1248 \\
\hline $\mathrm{J} 1835-1548$ & 0.670486 & -14.7595 & 15.04 & -10.2896 & -15.815 & -34.4933 \\
\hline J1836-0436 & 0.354237 & -14.7799 & 4.62 & -10.5871 & -15.5583 & -33.724 \\
\hline J1836-0517 & 0.457245 & -14.8861 & 8.08 & -10.5825 & -15.7754 & -34.1838 \\
\hline J1836-1008 & 0.562711 & -13.9281 & 5.39 & -9.53438 & -14.9076 & -33.1402 \\
\hline J1836-1324 & 0.178756 & -14.983 & 3.91 & -11.0873 & -15.4644 & -33.5576 \\
\hline J1837-0045 & 0.617037 & -14.7747 & 2.55 & -10.3409 & -15.7941 & -33.7017 \\
\hline $\mathrm{J} 1837+0053$ & 0.473513 & -16.4237 & 3.24 & -12.1049 & -17.3281 & -35.3397 \\
\hline J1837-0559 & 0.201063 & -14.4815 & 5.01 & -10.5347 & -15.014 & -33.2149 \\
\hline J1837-0604 & 0.096294 & -13.3449 & 6.19 & -9.71781 & -13.5576 & -31.8504 \\
\hline $\mathrm{J} 1837-0653$ & 1.90581 & -15.1124 & 4.93 & -10.1889 & -16.6216 & -34.8155 \\
\hline $\mathrm{J} 1837+1221$ & 1.96353 & -14.2076 & 6.1 & -9.27112 & -15.7298 & -34.0162 \\
\hline $\mathrm{J} 1837-1243$ & 1.87602 & -13.4377 & 7.51 & -8.52102 & -14.9401 & -33.3168 \\
\hline J1837-1837 & 0.618358 & -14.2596 & 3.04 & -9.82494 & -15.28 & -33.264 \\
\hline $\mathrm{J} 1838-0107$ & 0.444426 & -17.2628 & 5.83 & -12.9716 & -18.1398 & -36.4065 \\
\hline $\mathrm{J} 1838-0453$ & 0.380831 & -12.9355 & 8.28 & -8.71136 & -13.7454 & -32.1645 \\
\hline J1838-0549 & 0.235303 & -13.4763 & 4.73 & -9.46117 & -14.077 & -32.2529 \\
\hline J1838-0624 & 0.927178 & -16.1113 & 5.82 & -11.5006 & -17.3076 & -35.5736 \\
\hline $\mathrm{J} 1838-0655$ & 0.070498 & -13.308 & 6.6 & -9.81641 & -13.3854 & -31.706 \\
\hline $\mathrm{J} 1838-1046$ & 1.21835 & -14.5114 & 4.35 & -9.78222 & -15.8264 & -33.9659 \\
\hline $\mathrm{J} 1838+1650$ & 1.90197 & -14.5719 & 1.84 & -9.64921 & -16.0802 & -33.8461 \\
\hline J1838-1849 & 0.488242 & -16.3979 & 6.3 & -12.0659 & -17.3157 & -35.6161 \\
\hline $\mathrm{J} 1839-0223$ & 1.26679 & -14.3224 & 5.78 & -9.57624 & -15.6542 & -33.9172 \\
\hline J1839-0321 & 0.238778 & -13.9031 & 6.82 & -9.88164 & -14.5102 & -32.8451 \\
\hline J1839-0332 & 2.67568 & -14.3224 & 4.24 & -9.25151 & -15.979 & -34.1074 \\
\hline J1839-0402 & 0.52094 & -14.1141 & 4.69 & -9.75383 & -15.06 & -33.2322 \\
\hline J1839-0436 & 0.149461 & -15.0915 & 4.98 & -11.2735 & -15.4952 & -33.6935 \\
\hline
\end{tabular}


Table 1 continued

\begin{tabular}{|c|c|c|c|c|c|c|}
\hline Pulsar & $P(\mathrm{~s})$ & $\log \dot{P}$ & $d(\mathrm{kpc})$ & $\log \varepsilon$ & $\log \eta$ & $\log h$ \\
\hline J1839-0459 & 0.585319 & -14.4802 & 4.59 & -10.0693 & -15.4767 & -33.6396 \\
\hline J1839-0627 & 0.484914 & -15.8794 & 2.33 & -11.5503 & -16.7942 & -34.6627 \\
\hline $\mathrm{J} 1839-0643$ & 0.449548 & -14.4389 & 6.55 & -10.1427 & -15.3208 & -33.6381 \\
\hline J1839-0905 & 0.418969 & -13.585 & 6.14 & -9.31939 & -14.4364 & -32.7256 \\
\hline $\mathrm{J} 1839-1238$ & 1.91143 & -14.3054 & 4.18 & -9.38058 & -15.8159 & -33.9381 \\
\hline $\mathrm{J} 1839+15$ & 0.549161 & -13.5834 & 3.94 & -9.20021 & -14.5522 & -32.6488 \\
\hline $\mathrm{J} 1840+0214$ & 0.797478 & -14.0814 & 5.42 & -9.53627 & -15.2123 & -33.4474 \\
\hline J1840-0559 & 0.859368 & -14.0177 & 5.04 & -9.4401 & -15.1811 & -33.3845 \\
\hline $\mathrm{J} 1840-0626$ & 1.89335 & -13.6383 & 8.99 & -8.71759 & -15.1447 & -33.5995 \\
\hline $\mathrm{J} 1840-0643$ & 0.035578 & -15.6576 & 6.73 & -12.4629 & -15.4379 & -33.767 \\
\hline $\mathrm{J} 1840-0753$ & 0.437869 & -16 & 11.71 & -11.7152 & -16.8705 & -35.4401 \\
\hline J1840-0809 & 0.955672 & -14.6289 & 5.77 & -10.0052 & -15.8384 & -34.1006 \\
\hline $\mathrm{J} 1840-0815$ & 1.09644 & -14.6162 & 4.5 & -9.93275 & -15.8853 & -34.0396 \\
\hline $\mathrm{J} 1840-0840$ & 5.30938 & -13.6253 & 4.94 & -8.25676 & -15.5794 & -33.7742 \\
\hline $\mathrm{J} 1840-1122$ & 0.940962 & -14.1931 & 8.3 & -9.57612 & -15.3959 & -33.816 \\
\hline J1840-1207 & 0.75447 & -14.4949 & 9.59 & -9.97376 & -15.6016 & -34.0845 \\
\hline J1840-1419 & 6.59756 & -14.1986 & 1.01 & -8.73576 & -16.2471 & -33.7525 \\
\hline $\mathrm{J} 1840+5640$ & 1.65286 & -14.8268 & 2.61 & -9.96512 & -16.2742 & -34.1919 \\
\hline $\mathrm{J} 1841+0130$ & 0.029773 & -17.0878 & 3.19 & -13.9705 & -16.7907 & -34.7956 \\
\hline $\mathrm{J} 1841-0157$ & 0.663321 & -13.7423 & 7.27 & -9.27715 & -14.7932 & -33.1558 \\
\hline $\mathrm{J} 1841-0310$ & 1.65766 & -15.475 & 4.5 & -10.612 & -16.9236 & -35.0779 \\
\hline $\mathrm{J} 1841-0345$ & 0.204068 & -13.2373 & 4.15 & -9.28409 & -13.7762 & -31.8954 \\
\hline $\mathrm{J} 1841-0425$ & 0.186149 & -14.1945 & 5.17 & -10.2812 & -14.6935 & -32.9081 \\
\hline $\mathrm{J} 1841-0456$ & 11.789 & -10.3883 & 9.6 & -4.67335 & -12.6889 & -31.1722 \\
\hline $\mathrm{J} 1841-0500$ & 0.912914 & -13.4584 & 6.77 & -8.85454 & -14.648 & -32.9796 \\
\hline $\mathrm{J} 1841-0524$ & 0.445749 & -12.6308 & 4.89 & -8.33824 & -13.509 & -31.6994 \\
\hline $\mathrm{J} 1841+0912$ & 0.381319 & -14.9626 & 2.49 & -10.7378 & -15.773 & -33.6703 \\
\hline J1841-1404 & 1.33456 & -15.1931 & 10.85 & -10.4244 & -16.5476 & -35.0841 \\
\hline $\mathrm{J} 1841-7845$ & 0.353603 & -15.7959 & 4.01 & -11.6039 & -16.5735 & -34.6777 \\
\hline $\mathrm{J} 1842-0153$ & 1.05423 & -14.1726 & 6.49 & -9.50624 & -15.4247 & -33.738 \\
\hline $\mathrm{J} 1842+0257$ & 3.08826 & -13.5287 & 3.93 & -8.39554 & -15.2476 & -33.343 \\
\hline J1842-0309 & 0.40492 & -14.3449 & 11.41 & -10.094 & -15.1814 & -33.7397 \\
\hline $\mathrm{J} 1842+0358$ & 0.233326 & -15.091 & 3.21 & -11.0796 & -15.6881 & -33.6957 \\
\hline $\mathrm{J} 1842-0359$ & 1.83994 & -15.2933 & 4.15 & -10.385 & -16.7872 & -34.9063 \\
\hline $\mathrm{J} 1842-0415$ & 0.526682 & -13.6596 & 4.02 & -9.29455 & -14.6103 & -32.7155 \\
\hline J1842-0612 & 0.564475 & -16.6576 & 7.01 & -12.2625 & -17.6384 & -35.9851 \\
\hline $\mathrm{J} 1842+0638$ & 0.313016 & -16.1192 & 11.04 & -11.9802 & -16.8439 & -35.3879 \\
\hline $\mathrm{J} 1842-0800$ & 1.25547 & -15.7122 & 4.23 & -10.9699 & -17.0402 & -35.1676 \\
\hline $\mathrm{J} 1842-0905$ & 0.344643 & -13.9788 & 7.41 & -9.79799 & -14.7453 & -33.1162 \\
\hline $\mathrm{J} 1842+1332$ & 0.471604 & -15.6402 & 5.9 & -11.3231 & -16.5429 & -34.8148 \\
\hline $\mathrm{J} 1843-0000$ & 0.88033 & -14.1085 & 2.73 & -9.52036 & -15.2823 & -33.2195 \\
\hline $\mathrm{J} 1843-0050$ & 0.782598 & -15.6038 & 7.73 & -11.0668 & -16.7265 & -35.1157 \\
\hline J1843-0137 & 0.669872 & -14.6073 & 7.13 & -10.1379 & -15.6624 & -34.0166 \\
\hline $\mathrm{J} 1843-0211$ & 2.02752 & -13.8416 & 6.34 & -8.89122 & -15.3778 & -33.6809 \\
\hline $\mathrm{J} 1843-0355$ & 0.132314 & -14.983 & 8.81 & -11.2179 & -15.3337 & -33.7798 \\
\hline $\mathrm{J} 1843-0408$ & 0.781934 & -14.6216 & 4.68 & -10.085 & -15.7439 & -33.9152 \\
\hline J1843-0459 & 0.754963 & -15.0685 & 6.15 & -10.5472 & -16.1756 & -34.4655 \\
\hline
\end{tabular}


Table 1 continued

\begin{tabular}{|c|c|c|c|c|c|c|}
\hline Pulsar & $P(\mathrm{~s})$ & $\log \dot{P}$ & $d(\mathrm{kpc})$ & $\log \varepsilon$ & $\log \eta$ & $\log h$ \\
\hline $\mathrm{J} 1843-0510$ & 0.671614 & -14.4101 & 4.72 & -9.93948 & -15.4663 & -33.6413 \\
\hline $\mathrm{J} 1843-0702$ & 0.191614 & -14.6696 & 4.57 & -10.7437 & -15.1812 & -33.3421 \\
\hline $\mathrm{J} 1843-0744$ & 0.475393 & -13.8761 & 5.77 & -9.55564 & -14.7823 & -33.0446 \\
\hline J1843-0806 & 0.536414 & -13.7595 & 4.55 & -9.3865 & -14.7181 & -32.8772 \\
\hline $\mathrm{J} 1843-1113$ & 0.001846 & -20.0182 & 1.97 & -18.1085 & -18.5136 & -36.3091 \\
\hline J1843-1448 & 0.005471 & -20.2069 & 3.42 & -17.8254 & -19.1741 & -37.2092 \\
\hline $\mathrm{J} 1843-1507$ & 0.58355 & -14.1427 & 9.05 & -9.73314 & -15.1379 & -33.5956 \\
\hline $\mathrm{J} 1843+2024$ & 3.40654 & -14.983 & 7.72 & -9.8072 & -16.7444 & -35.1331 \\
\hline $\mathrm{J} 1844-0030$ & 0.641098 & -14.2161 & 8.66 & -9.76572 & -15.2522 & -33.6907 \\
\hline $\mathrm{J} 1844+0115$ & 0.004186 & -19.9706 & 3.45 & -17.7054 & -18.8216 & -36.8604 \\
\hline $\mathrm{J} 1844-0244$ & 0.507722 & -13.7773 & 5.99 & -9.4282 & -14.7121 & -32.9905 \\
\hline $\mathrm{J} 1844-0302$ & 1.19863 & -14.1073 & 6.8 & -9.38521 & -15.4152 & -33.7487 \\
\hline $\mathrm{J} 1844-0310$ & 0.525049 & -13.9914 & 8.88 & -9.62775 & -14.9407 & -33.3902 \\
\hline $\mathrm{J} 1844-0433$ & 0.991027 & -14.4078 & 2.78 & -9.76829 & -15.6331 & -33.5782 \\
\hline $\mathrm{J} 1844-0452$ & 0.269443 & -15.1675 & 8.06 & -11.0936 & -15.8271 & -34.2345 \\
\hline $\mathrm{J} 1844-0502$ & 0.335163 & -16.209 & 5.2 & -12.0403 & -16.9634 & -35.1805 \\
\hline $\mathrm{J} 1844-0538$ & 0.255699 & -14.0128 & 6.18 & -9.9616 & -14.6497 & -32.9417 \\
\hline $\mathrm{J} 1844+1454$ & 0.375463 & -14.7282 & 2.61 & -10.5101 & -15.5319 & -33.4496 \\
\hline $\mathrm{J} 1845-0316$ & 0.207636 & -14.0526 & 6.53 & -10.0918 & -14.599 & -32.915 \\
\hline $\mathrm{J} 1845-0434$ & 0.486751 & -13.9469 & 4.61 & -9.61616 & -14.8634 & -33.0281 \\
\hline $\mathrm{J} 1845-0545$ & 1.09235 & -13.8729 & 5.39 & -9.19108 & -15.1404 & -33.3731 \\
\hline $\mathrm{J} 1845+0623$ & 1.42165 & -15.2628 & 3.53 & -10.4666 & -16.6447 & -34.6936 \\
\hline $\mathrm{J} 1845-0635$ & 0.340528 & -14.3478 & 7.56 & -10.1721 & -15.1091 & -33.4886 \\
\hline $\mathrm{J} 1845-0743$ & 0.104695 & -15.4353 & 5.85 & -11.772 & -15.6844 & -33.9526 \\
\hline $\mathrm{J} 1845-0826$ & 0.634354 & -14.0292 & 4.87 & -9.5834 & -15.0607 & -33.2493 \\
\hline $\mathrm{J} 1845-1114$ & 0.20622 & -14.6968 & 5.82 & -10.739 & -15.2403 & -33.5063 \\
\hline $\mathrm{J} 1845-1351$ & 2.61892 & -14.0123 & 7.1 & -8.95076 & -15.6596 & -34.0119 \\
\hline $\mathrm{J} 1846+0051$ & 0.434373 & -13.9508 & 3.2 & -9.66947 & -14.8178 & -32.824 \\
\hline $\mathrm{J} 1846-0257$ & 4.47672 & -12.7932 & 4.69 & -7.49876 & -14.6733 & -32.8455 \\
\hline $\mathrm{J} 1846-0258$ & 0.326571 & -11.1481 & 5.8 & -6.9907 & -11.8913 & -30.1557 \\
\hline J1846-0749 & 0.35011 & -14.8996 & 10.1 & -10.712 & -15.673 & -34.1784 \\
\hline J1846-07492 & 0.86138 & -14.2848 & 4.47 & -9.70619 & -15.4492 & -33.6005 \\
\hline J1846-7403 & 4.87884 & -14.2175 & 4.07 & -8.88576 & -16.135 & -34.2456 \\
\hline $\mathrm{J} 1847-0130$ & 6.70705 & -11.8962 & 7.64 & -6.42621 & -13.9519 & -32.336 \\
\hline $\mathrm{J} 1847-0402$ & 0.597769 & -13.2865 & 3.12 & -8.86652 & -14.2922 & -32.2874 \\
\hline $\mathrm{J} 1847-0427$ & 0.259247 & -17.2291 & 4.17 & -13.172 & -17.872 & -35.9932 \\
\hline $\mathrm{J} 1847-0438$ & 0.957991 & -13.9626 & 4.72 & -9.33776 & -15.1731 & -33.3481 \\
\hline $\mathrm{J} 1847-0443$ & 0.340832 & -16.5482 & 7.57 & -12.3722 & -17.3099 & -35.6901 \\
\hline $\mathrm{J} 1847-0605$ & 0.778164 & -14.3325 & 4.59 & -9.79802 & -15.4528 & -33.6156 \\
\hline $\mathrm{J} 1848-0023$ & 0.537624 & -14.7932 & 1.55 & -10.4192 & -15.7528 & -33.4442 \\
\hline $\mathrm{J} 1848-0055$ & 0.274557 & -14.8697 & 15.15 & -10.7876 & -15.5374 & -34.2189 \\
\hline $\mathrm{J} 1848-0123$ & 0.659432 & -14.2798 & 4.4 & -9.81722 & -15.3282 & -33.4727 \\
\hline $\mathrm{J} 1848+0351$ & 0.191443 & -16.172 & 10.53 & -12.2465 & -16.6832 & -35.2067 \\
\hline $\mathrm{J} 1848-0511$ & 1.63713 & -14.0526 & 7.78 & -9.19503 & -15.4958 & -33.8878 \\
\hline $\mathrm{J} 1848-0601$ & 0.225004 & -15.5421 & 12.13 & -11.5465 & -16.1235 & -34.7084 \\
\hline $\mathrm{J} 1848+0604$ & 2.2186 & -14.4271 & 8.78 & -9.4376 & -16.0024 & -34.4469 \\
\hline $\mathrm{J} 1848+0647$ & 0.505957 & -14.058 & 1.44 & -9.71043 & -14.9913 & -32.6507 \\
\hline
\end{tabular}


Table 1 continued

\begin{tabular}{|c|c|c|c|c|c|c|}
\hline Pulsar & $P(\mathrm{~s})$ & $\log \dot{P}$ & $d(\mathrm{kpc})$ & $\log \varepsilon$ & $\log \eta$ & $\log h$ \\
\hline $\mathrm{J} 1848+0826$ & 0.328665 & -15.5654 & 3.37 & -11.4052 & -16.3113 & -34.34 \\
\hline $\mathrm{J} 1848-1150$ & 1.31222 & -14.8447 & 4.77 & -10.0832 & -16.1918 & -34.3714 \\
\hline J1848-1414 & 0.29777 & -16.8508 & 4.39 & -12.7334 & -17.5538 & -35.6973 \\
\hline J1848-1952 & 4.30819 & -13.6326 & 0.95 & -8.3549 & -15.4961 & -32.9749 \\
\hline $\mathrm{J} 1849-0040$ & 0.672481 & -13.9547 & 20.86 & -9.48354 & -15.0115 & -33.8319 \\
\hline $\mathrm{J} 1849+0127$ & 0.542155 & -13.5528 & 4.54 & -9.17527 & -14.5161 & -32.6742 \\
\hline J1849-0317 & 0.668408 & -13.6576 & 1.9 & -9.18908 & -14.7118 & -32.4916 \\
\hline $\mathrm{J} 1849+0409$ & 0.761194 & -13.6655 & 2.41 & -9.1406 & -14.7762 & -32.6593 \\
\hline J1849-0614 & 0.953384 & -13.2684 & 2.84 & -8.64569 & -14.4768 & -32.4312 \\
\hline $\mathrm{J} 1849-0636$ & 1.45132 & -13.3354 & 3.68 & -8.53014 & -14.7263 & -32.7932 \\
\hline $\mathrm{J} 1849+2423$ & 0.275641 & -15.8153 & 4.02 & -11.7315 & -16.4848 & -34.5901 \\
\hline $\mathrm{J} 1850-0006$ & 2.1915 & -14.3645 & 7.21 & -9.38032 & -15.9344 & -34.2934 \\
\hline $\mathrm{J} 1850-0026$ & 0.166634 & -13.4078 & 10.69 & -9.54261 & -13.8587 & -32.3888 \\
\hline $\mathrm{J} 1850+0026$ & 1.08184 & -15.4449 & 4.27 & -10.7673 & -16.7082 & -34.8397 \\
\hline $\mathrm{J} 1850-0031$ & 0.734185 & -14.8996 & 9.98 & -10.3904 & -15.9946 & -34.4948 \\
\hline $\mathrm{J} 1850+0124$ & 0.00356 & -19.9626 & 2.97 & -17.7677 & -18.7432 & -36.717 \\
\hline $\mathrm{J} 1850+0242$ & 0.00448 & -18.7878 & 13.1 & -16.4931 & -17.6682 & -36.2866 \\
\hline $\mathrm{J} 1850+0423$ & 0.290716 & -16.0391 & 7.38 & -11.9321 & -16.7317 & -35.1008 \\
\hline $\mathrm{J} 1850+1335$ & 0.345582 & -14.8268 & 3.14 & -10.6448 & -15.5945 & -33.5925 \\
\hline J1851-0029 & 0.518721 & -14.3242 & 6.86 & -9.96584 & -15.2683 & -33.6057 \\
\hline $\mathrm{J} 1851-0053$ & 1.40907 & -15.0585 & 1.24 & -10.2661 & -16.4366 & -34.031 \\
\hline J1851-0114 & 0.953182 & -14.6055 & 6.28 & -9.98292 & -15.8139 & -34.1129 \\
\hline $\mathrm{J} 1851+0118$ & 0.906977 & -12.8633 & 6.75 & -8.26223 & -14.05 & -32.3804 \\
\hline $\mathrm{J} 1851-0241$ & 0.435194 & -14.0991 & 7.62 & -9.81695 & -14.9669 & -33.3499 \\
\hline $\mathrm{J} 1851+0418$ & 0.284697 & -14.9626 & 3.17 & -10.8647 & -15.6461 & -33.6482 \\
\hline $\mathrm{J} 1851+1259$ & 1.2053 & -13.9393 & 3.5 & -9.21475 & -15.2495 & -33.2947 \\
\hline $\mathrm{J} 1852+0008$ & 0.467894 & -14.2457 & 5.1 & -9.93205 & -15.1449 & -33.3536 \\
\hline $\mathrm{J} 1852+0013$ & 0.957751 & -13.8539 & 7.22 & -9.22917 & -15.0643 & -33.4239 \\
\hline $\mathrm{J} 1852+0031$ & 2.18019 & -13.0132 & 8 & -8.03128 & -14.5809 & -32.985 \\
\hline $\mathrm{J} 1852+0040$ & 0.104913 & -17.0615 & 10 & -13.3972 & -17.3115 & -35.8125 \\
\hline J1852-0118 & 0.451473 & -14.7545 & 5.4 & -10.4564 & -15.6383 & -33.8717 \\
\hline $\mathrm{J} 1852-0127$ & 0.428979 & -14.2882 & 6.43 & -10.0123 & -15.1498 & -33.459 \\
\hline $\mathrm{J} 1852+0305$ & 1.32615 & -15.9914 & 6.73 & -11.2254 & -17.3431 & -35.6722 \\
\hline $\mathrm{J} 1852-0635$ & 0.524151 & -13.8356 & 4.6 & -9.47274 & -14.7843 & -32.9481 \\
\hline $\mathrm{J} 1852-2610$ & 0.336337 & -16.057 & 2.26 & -11.8868 & -16.8129 & -34.6681 \\
\hline J1853-0004 & 0.101436 & -14.2541 & 6.58 & -10.6045 & -14.4895 & -32.8088 \\
\hline $\mathrm{J} 1853+0011$ & 0.397882 & -13.475 & 7.48 & -9.23175 & -14.3039 & -32.6788 \\
\hline $\mathrm{J} 1853+0056$ & 0.275578 & -13.6696 & 3.82 & -9.58589 & -14.339 & -32.4221 \\
\hline $\mathrm{J} 1853+0505$ & 0.905137 & -14.8928 & 7.54 & -10.2926 & -16.0787 & -34.4571 \\
\hline $\mathrm{J} 1853+0545$ & 0.1264 & -15.2132 & 4.77 & -11.468 & -15.5441 & -33.7237 \\
\hline J1853-0649 & 1.04813 & -14.8097 & 1.89 & -10.1458 & -16.0592 & -33.8368 \\
\hline $\mathrm{J} 1853+0853$ & 3.91466 & -14.2899 & 7.54 & -9.05374 & -16.1117 & -34.4902 \\
\hline $\mathrm{J} 1853+1303$ & 0.004092 & -20.0595 & 1.6 & -17.8041 & -18.9006 & -36.6057 \\
\hline $\mathrm{J} 1854+0306$ & 4.55782 & -12.8386 & 4.11 & -7.53642 & -14.7265 & -32.8414 \\
\hline $\mathrm{J} 1854+0317$ & 1.36645 & -14.7328 & 7.95 & -9.95378 & -16.0976 & -34.499 \\
\hline $\mathrm{J} 1854+1050$ & 0.573197 & -15.1945 & 8.27 & -10.7927 & -16.182 & -34.6005 \\
\hline J1854-1421 & 1.1466 & -14.3809 & 5.23 & -9.67804 & -15.6695 & -33.889 \\
\hline
\end{tabular}


Table 1 continued

\begin{tabular}{|c|c|c|c|c|c|c|}
\hline Pulsar & $P(\mathrm{~s})$ & $\log \dot{P}$ & $d(\mathrm{kpc})$ & $\log \varepsilon$ & $\log \eta$ & $\log h$ \\
\hline J1854-1557 & 3.4532 & -14.3449 & 11.75 & -9.16319 & -16.1122 & -34.6833 \\
\hline $\mathrm{J} 1855+0205$ & 0.246817 & -16.1891 & 16.48 & -12.1533 & -16.8106 & -35.5286 \\
\hline $\mathrm{J} 1855+0307$ & 0.845348 & -13.7423 & 7.45 & -9.17183 & -14.8985 & -33.2717 \\
\hline $\mathrm{J} 1855+0422$ & 1.67811 & -15.032 & 9.85 & -10.1637 & -16.486 & -34.9804 \\
\hline $\mathrm{J} 1855+0527$ & 1.39348 & -12.5735 & 9.7 & -7.78594 & -13.9467 & -32.4346 \\
\hline $\mathrm{J} 1855+0700$ & 0.258685 & -15.1238 & 6.84 & -11.0676 & -15.7657 & -34.1018 \\
\hline J1855-0941 & 0.345401 & -15.6198 & 4.87 & -11.438 & -16.3873 & -34.5758 \\
\hline $\mathrm{J} 1856+0102$ & 0.620217 & -14.9136 & 8.61 & -10.4776 & -15.9353 & -34.3714 \\
\hline $\mathrm{J} 1856+0113$ & 0.26744 & -12.6819 & 3.3 & -8.61126 & -13.3383 & -31.3579 \\
\hline $\mathrm{J} 1856+0245$ & 0.080907 & -13.2069 & 10.29 & -9.65547 & -13.344 & -31.8575 \\
\hline $\mathrm{J} 1856+0404$ & 0.420252 & -16.4306 & 6.98 & -12.1637 & -17.2833 & -35.6282 \\
\hline $\mathrm{J} 1856-0526$ & 0.370483 & -14.7696 & 3.45 & -10.5573 & -15.5675 & -33.6063 \\
\hline J1856-3754 & 7.0552 & -13.5258 & 0.16 & -8.03382 & -15.6034 & -32.3086 \\
\hline $\mathrm{J} 1857+0057$ & 0.356929 & -16.2628 & 2.63 & -12.0668 & -17.0445 & -34.9656 \\
\hline $\mathrm{J} 1857+0143$ & 0.13976 & -13.5058 & 5.18 & -9.71701 & -13.8804 & -32.0958 \\
\hline $\mathrm{J} 1857+0210$ & 0.630983 & -13.8539 & 15.01 & -9.4104 & -14.883 & -33.5605 \\
\hline $\mathrm{J} 1857+0212$ & 0.415823 & -13.3947 & 8 & -9.13233 & -14.2428 & -32.6469 \\
\hline $\mathrm{J} 1857+0526$ & 0.349951 & -14.1593 & 11.44 & -9.97181 & -14.9324 & -33.4919 \\
\hline $\mathrm{J} 1857+0809$ & 0.502924 & -14.3242 & 8.72 & -9.97927 & -15.2549 & -33.6964 \\
\hline $\mathrm{J} 1857+0943$ & 0.005362 & -19.7496 & 0.7 & -17.3768 & -18.7081 & -36.0542 \\
\hline J1857-1027 & 3.68722 & -13.9788 & 3.64 & -8.76866 & -15.7747 & -33.8368 \\
\hline $\mathrm{J} 1858+0215$ & 0.745828 & -14.3363 & 12.46 & -9.82021 & -15.4381 & -34.0347 \\
\hline $\mathrm{J} 1858+0241$ & 4.69323 & -13.6144 & 6.46 & -8.29947 & -15.515 & -33.8263 \\
\hline $\mathrm{J} 1858+0346$ & 0.256844 & -14.6904 & 7.28 & -10.6372 & -15.3292 & -33.6924 \\
\hline J1858-0736 & 0.551059 & -14.2958 & 7.2 & -9.9112 & -15.2662 & -33.6246 \\
\hline $\mathrm{J} 1859+0601$ & 1.04431 & -13.5935 & 5.98 & -8.93118 & -14.8414 & -33.1192 \\
\hline $\mathrm{J} 1859+1526$ & 0.933972 & -14.4056 & 4.76 & -9.79182 & -15.6051 & -33.7838 \\
\hline $\mathrm{J} 1900-0051$ & 0.385194 & -15.8477 & 3.3 & -11.6186 & -16.6625 & -34.6821 \\
\hline $\mathrm{J} 1900+0227$ & 0.374262 & -14.2434 & 4.32 & -10.0267 & -15.0457 & -33.1822 \\
\hline $\mathrm{J} 1900+0308$ & 0.004909 & -20.2291 & 5.27 & -17.8947 & -19.1493 & -37.3722 \\
\hline $\mathrm{J} 1900+0634$ & 0.389869 & -14.2899 & 7.31 & -10.0555 & -15.1099 & -33.4749 \\
\hline $\mathrm{J} 1900-2600$ & 0.612209 & -15.6882 & 0.7 & -11.2579 & -16.7043 & -34.0505 \\
\hline J1900-7951 & 1.27919 & -14.7305 & 3.87 & -9.9801 & -16.0666 & -34.1553 \\
\hline $\mathrm{J} 1901+0124$ & 0.318817 & -14.4895 & 7.2 & -10.3425 & -15.2221 & -33.5805 \\
\hline $\mathrm{J} 1901+0156$ & 0.288219 & -14.6271 & 2.96 & -10.5239 & -15.316 & -33.2883 \\
\hline $\mathrm{J} 1901+0254$ & 1.29969 & -15.341 & 3.95 & -10.5837 & -16.684 & -34.7817 \\
\hline $\mathrm{J} 1901+0300$ & 0.007797 & -19.3401 & 5.5 & -16.8047 & -18.4612 & -36.7026 \\
\hline $\mathrm{J} 1901-0312$ & 0.355725 & -14.6402 & 2.89 & -10.4456 & -15.4204 & -33.3824 \\
\hline J1901-0315 & 0.801693 & -14.5901 & 6.95 & -10.0426 & -15.7232 & -34.0663 \\
\hline $\mathrm{J} 1901+0320$ & 0.636584 & -15.2815 & 7.74 & -10.8342 & -16.3145 & -34.7043 \\
\hline $\mathrm{J} 1901+0331$ & 0.65545 & -14.1273 & 7 & -9.66727 & -15.1729 & -33.5191 \\
\hline $\mathrm{J} 1901+0355$ & 0.554756 & -13.8962 & 10.18 & -9.50864 & -14.8694 & -33.3783 \\
\hline $\mathrm{J} 1901+0413$ & 2.66308 & -12.8794 & 6.91 & -7.81059 & -14.534 & -32.8745 \\
\hline $\mathrm{J} 1901+0435$ & 0.690576 & -14.062 & 30 & -9.57932 & -15.1303 & -34.1085 \\
\hline $\mathrm{J} 1901+0510$ & 0.614757 & -13.5072 & 8.47 & -9.07508 & -14.5251 & -32.954 \\
\hline $\mathrm{J} 1901+0621$ & 0.832002 & -16.7447 & 3.11 & -12.1812 & -17.894 & -35.8878 \\
\hline $\mathrm{J} 1901+0716$ & 0.643999 & -14.6402 & 3.4 & -10.1878 & -15.6782 & -33.7107 \\
\hline
\end{tabular}


Table 1 continued

\begin{tabular}{|c|c|c|c|c|c|c|}
\hline Pulsar & $P(\mathrm{~s})$ & $\log \dot{P}$ & $d(\mathrm{kpc})$ & $\log \varepsilon$ & $\log \eta$ & $\log h$ \\
\hline J1901-0906 & 1.78193 & -14.7852 & 2.42 & -9.89081 & -16.2652 & -34.1501 \\
\hline $\mathrm{J} 1901+1306$ & 1.83086 & -15.8827 & 3.53 & -10.9766 & -17.3745 & -35.4234 \\
\hline $\mathrm{J} 1901-1740$ & 1.95686 & -15.0846 & 1.27 & -10.1496 & -16.6053 & -34.2102 \\
\hline $\mathrm{J} 1902+0248$ & 1.22378 & -14.618 & 6.09 & -9.88683 & -15.9348 & -34.2205 \\
\hline J1902-0340 & 1.52467 & -14.699 & 3.13 & -9.87234 & -16.1113 & -34.1079 \\
\hline $\mathrm{J} 1902+0556$ & 0.746577 & -13.8894 & 3.6 & -9.37288 & -14.9916 & -33.049 \\
\hline $\mathrm{J} 1902+0615$ & 0.673503 & -14.1129 & 7 & -9.64115 & -15.1704 & -33.5166 \\
\hline $\mathrm{J} 1902+0723$ & 0.487813 & -15.6778 & 3.33 & -11.3461 & -16.5952 & -34.6187 \\
\hline J1902-5105 & 0.001742 & -20.0362 & 2.1 & -18.1517 & -18.5064 & -36.3297 \\
\hline $\mathrm{J} 1903+0135$ & 0.729304 & -14.3947 & 3.3 & -9.88833 & -15.4868 & -33.5063 \\
\hline $\mathrm{J} 1903-0258$ & 0.301459 & -15.1681 & 3.1 & -11.0454 & -15.8765 & -33.8689 \\
\hline $\mathrm{J} 1903+0327$ & 0.00215 & -19.7258 & 6.45 & -17.75 & -18.2874 & -36.598 \\
\hline $\mathrm{J} 1903+0601$ & 0.374117 & -13.7167 & 7.84 & -9.50024 & -14.5189 & -32.9142 \\
\hline J1903-0632 & 0.431887 & -14.4685 & 8.77 & -10.1897 & -15.333 & -33.7771 \\
\hline J1903-0848 & 0.887325 & -14.8761 & 2.32 & -10.2846 & -16.0534 & -33.9199 \\
\hline $\mathrm{J} 1903+0925$ & 0.357155 & -13.433 & 4.17 & -9.23666 & -14.215 & -32.3362 \\
\hline $\mathrm{J} 1903+2225$ & 0.651185 & -15.3516 & 7.86 & -10.8945 & -16.3945 & -34.791 \\
\hline J1903-7051 & 0.003598 & -19.983 & 1.13 & -17.7835 & -18.7682 & -36.3223 \\
\hline J1904+0004 & 0.139525 & -15.9281 & 6.43 & -12.14 & -16.3019 & -34.6112 \\
\hline J1904-0150 & 0.379387 & -15.0506 & 4.93 & -10.8281 & -15.8588 & -34.0527 \\
\hline J1904+0412 & 0.071095 & -18.9586 & 4.01 & -15.4633 & -19.0396 & -37.1438 \\
\hline $\mathrm{J} 1904+0451$ & 0.006092 & -20.2426 & 3.96 & -17.8144 & -19.2565 & -37.3553 \\
\hline J1904+0738 & 0.208958 & -15.3862 & 5.86 & -11.4226 & -15.9354 & -34.2043 \\
\hline $\mathrm{J} 1904+0800$ & 0.263345 & -13.762 & 9.2 & -9.69798 & -14.4116 & -32.8765 \\
\hline J1904+1011 & 1.85657 & -15.5591 & 4.03 & -10.6469 & -17.057 & -35.1633 \\
\hline J1904-1224 & 0.750808 & -15.1296 & 5.63 & -10.6106 & -16.2343 & -34.4858 \\
\hline J1905-0056 & 0.643181 & -14.5157 & 6.91 & -10.0639 & -15.5532 & -33.8937 \\
\hline $\mathrm{J} 1905+0400$ & 0.003784 & -20.3089 & 1.33 & -18.0875 & -19.116 & -36.7409 \\
\hline $\mathrm{J} 1905+0600$ & 0.44121 & -14.9547 & 18.06 & -10.6666 & -15.8285 & -34.5862 \\
\hline $\mathrm{J} 1905+0616$ & 0.989706 & -12.8697 & 5.24 & -8.23071 & -14.0943 & -32.3147 \\
\hline $\mathrm{J} 1905+0709$ & 0.64804 & -14.3063 & 4.98 & -9.85122 & -15.347 & -33.5453 \\
\hline $\mathrm{J} 1905+0902$ & 0.218253 & -14.4559 & 9.14 & -10.4735 & -15.024 & -33.486 \\
\hline J1906+0414 & 1.04336 & -13.9393 & 9.21 & -9.27742 & -15.1869 & -33.6522 \\
\hline J1906+0641 & 0.267275 & -14.6696 & 7 & -10.5992 & -15.3257 & -33.6718 \\
\hline $\mathrm{J} 1906+0649$ & 1.28656 & -15.8182 & 5.07 & -11.0653 & -17.1567 & -35.3628 \\
\hline $\mathrm{J} 1906+0746$ & 0.144073 & -13.6925 & 7.4 & -9.89047 & -14.0802 & -32.4505 \\
\hline J1906+0912 & 0.775345 & -15.8794 & 5.63 & -11.3465 & -16.9981 & -35.2496 \\
\hline J1906+1854 & 1.01909 & -15.6882 & 11.06 & -11.0366 & -16.9256 & -35.4704 \\
\hline $\mathrm{J} 1907+0249$ & 0.351879 & -14.9431 & 7.49 & -10.7532 & -15.7186 & -34.0942 \\
\hline $\mathrm{J} 1907+0345$ & 0.240153 & -14.0851 & 8.62 & -10.0612 & -14.6948 & -33.1313 \\
\hline $\mathrm{J} 1907+0534$ & 1.1384 & -14.5017 & 11.94 & -9.80194 & -15.7871 & -34.3652 \\
\hline $\mathrm{J} 1907+0602$ & 0.106633 & -13.0615 & 3.01 & -9.39014 & -13.3185 & -31.2981 \\
\hline $\mathrm{J} 1907+0731$ & 0.363676 & -13.7352 & 4.89 & -9.53101 & -14.525 & -32.7154 \\
\hline $\mathrm{J} 1907+0740$ & 0.574698 & -15.1733 & 6.84 & -10.7704 & -16.1619 & -34.498 \\
\hline $\mathrm{J} 1907+0918$ & 0.226107 & -13.0255 & 7.68 & -9.02772 & -13.609 & -31.9954 \\
\hline J1907+1149 & 1.42016 & -12.7959 & 5.28 & -8.00009 & -14.1774 & -32.4011 \\
\hline $\mathrm{J} 1907+1247$ & 0.827097 & -14.71 & 7.18 & -10.149 & -15.8567 & -34.2139 \\
\hline
\end{tabular}


Table 1 continued

\begin{tabular}{|c|c|c|c|c|c|c|}
\hline Pulsar & $P(\mathrm{~s})$ & $\log \dot{P}$ & $d(\mathrm{kpc})$ & $\log \varepsilon$ & $\log \eta$ & $\log h$ \\
\hline J1907-1532 & 0.632235 & -14.5114 & 3.09 & -10.0671 & -15.5415 & -33.5325 \\
\hline $\mathrm{J} 1907+4002$ & 1.23576 & -15.2668 & 1.76 & -10.5314 & -16.5879 & -34.3345 \\
\hline $\mathrm{J} 1908+0457$ & 0.846793 & -15.0088 & 9.3 & -10.4375 & -16.1657 & -34.6352 \\
\hline $\mathrm{J} 1908+0500$ & 0.291021 & -14.5867 & 4.43 & -10.4793 & -15.2798 & -33.4272 \\
\hline $\mathrm{J} 1908+0734$ & 0.212353 & -15.0835 & 0.58 & -11.113 & -15.6398 & -32.9042 \\
\hline J1908+0839 & 0.185397 & -14.6216 & 9.42 & -10.71 & -15.1189 & -33.594 \\
\hline $\mathrm{J} 1908+0909$ & 0.336555 & -13.4572 & 8.81 & -9.28667 & -14.2134 & -32.6594 \\
\hline $\mathrm{J} 1908+0916$ & 0.83027 & -16.0088 & 5.17 & -11.4461 & -17.1571 & -35.3717 \\
\hline $\mathrm{J} 1908+2351$ & 0.377578 & -16.7696 & 6.63 & -12.5491 & -17.5757 & -35.8983 \\
\hline $\mathrm{J} 1909+0007$ & 1.01695 & -14.2581 & 3.26 & -9.60731 & -15.4945 & -33.5088 \\
\hline $\mathrm{J} 1909+0254$ & 0.989831 & -14.2573 & 4.5 & -9.61826 & -15.482 & -33.6363 \\
\hline $\mathrm{J} 1909+0616$ & 0.755993 & -13.6861 & 8.59 & -9.16416 & -14.7938 & -33.2289 \\
\hline J1909+0641 & 0.741762 & -14.4921 & 1.86 & -9.97843 & -15.5916 & -33.3621 \\
\hline J1909+0749 & 0.237161 & -12.8182 & 10.84 & -8.79966 & -13.4223 & -31.9584 \\
\hline $\mathrm{J} 1909+0912$ & 0.222949 & -13.4461 & 8.2 & -9.45446 & -14.0235 & -32.4383 \\
\hline $\mathrm{J} 1909+1102$ & 0.283641 & -14.5784 & 4.8 & -10.4822 & -15.2603 & -33.4426 \\
\hline $\mathrm{J} 1909+1450$ & 0.996108 & -15.6596 & 4.94 & -11.0178 & -16.887 & -35.0818 \\
\hline J1909+1859 & 0.542451 & -16.0132 & 3.4 & -11.6354 & -16.9767 & -35.0093 \\
\hline J1909-3744 & 0.002947 & -19.8539 & 1.14 & -17.741 & -18.5524 & -36.1104 \\
\hline J1910-0112 & 1.3606 & -15.7496 & 6.44 & -10.9724 & -17.1125 & -35.4224 \\
\hline $\mathrm{J} 1910+0225$ & 0.337855 & -15.5817 & 6.33 & -11.4095 & -16.3396 & -34.642 \\
\hline J1910-0309 & 0.504605 & -14.6596 & 10.25 & -10.3132 & -15.5917 & -34.1034 \\
\hline $\mathrm{J} 1910+0358$ & 2.33026 & -14.3497 & 2.95 & -9.33884 & -15.9462 & -33.9171 \\
\hline $\mathrm{J} 1910+0534$ & 0.452867 & -14.7167 & 15.39 & -10.4173 & -15.6018 & -34.2901 \\
\hline $\mathrm{J} 1910+0714$ & 2.71242 & -14.2132 & 3.6 & -9.13644 & -15.8758 & -33.9331 \\
\hline $\mathrm{J} 1910+0728$ & 0.325415 & -14.0804 & 6.04 & -9.92451 & -14.822 & -33.1041 \\
\hline $\mathrm{J} 1910+1231$ & 1.44174 & -14.0846 & 6.47 & -9.28226 & -15.4726 & -33.7846 \\
\hline $\mathrm{J} 1910+1256$ & 0.004984 & -20.0137 & 1.95 & -17.6726 & -18.9404 & -36.7315 \\
\hline J1911-1114 & 0.003626 & -19.8477 & 1.59 & -17.6448 & -18.6363 & -36.3387 \\
\hline $\mathrm{J} 1911+1347$ & 0.004626 & -19.767 & 1.6 & -17.4583 & -18.6614 & -36.3665 \\
\hline $\mathrm{J} 1911+1758$ & 0.460406 & -16.8827 & 2.54 & -12.5761 & -17.775 & -35.6809 \\
\hline $\mathrm{J} 1912+1036$ & 0.409349 & -13.8013 & 4.23 & -9.5458 & -14.6426 & -32.77 \\
\hline $\mathrm{J} 1912+2104$ & 2.23297 & -13.9914 & 4.84 & -8.99906 & -15.5694 & -33.7553 \\
\hline $\mathrm{J} 1912+2525$ & 0.621976 & -15.6478 & 2.02 & -11.2106 & -16.6707 & -34.4771 \\
\hline J1913-0440 & 0.825936 & -14.3904 & 3.22 & -9.83001 & -15.5365 & -33.5454 \\
\hline $\mathrm{J} 1913+0446$ & 1.61613 & -12.5544 & 3.41 & -7.70247 & -13.992 & -32.0258 \\
\hline $\mathrm{J} 1913+0832$ & 0.134409 & -14.3401 & 7.76 & -10.5682 & -14.6977 & -33.0886 \\
\hline $\mathrm{J} 1913+0904$ & 0.163246 & -13.7545 & 3.49 & -9.89819 & -14.1965 & -32.2404 \\
\hline $\mathrm{J} 1913+0936$ & 1.24196 & -15.3645 & 4.22 & -10.627 & -16.6878 & -34.8141 \\
\hline $\mathrm{J} 1913+1000$ & 0.837148 & -13.7773 & 7.89 & -9.21103 & -14.9292 & -33.3274 \\
\hline $\mathrm{J} 1913+1011$ & 0.035909 & -14.4724 & 4.48 & -11.2737 & -14.2567 & -32.4091 \\
\hline $\mathrm{J} 1913+1145$ & 0.306069 & -14.2993 & 15.06 & -10.17 & -15.0143 & -33.6931 \\
\hline $\mathrm{J} 1913+1330$ & 0.923391 & -14.0615 & 5.35 & -9.45264 & -15.256 & -33.4854 \\
\hline $\mathrm{J} 1913+1400$ & 0.521473 & -15.0947 & 5.07 & -10.7341 & -16.0411 & -34.2472 \\
\hline $\mathrm{J} 1913+3732$ & 0.851079 & -14.8601 & 5.5 & -10.2867 & -16.0192 & -34.2607 \\
\hline $\mathrm{J} 1914+0219$ & 0.457527 & -14.9914 & 9.77 & -10.6875 & -15.881 & -34.3719 \\
\hline $\mathrm{J} 1914+0631$ & 0.693811 & -16.4789 & 2.68 & -11.9942 & -17.5493 & -35.4784 \\
\hline
\end{tabular}


Table 1 continued

\begin{tabular}{|c|c|c|c|c|c|c|}
\hline Pulsar & $P(\mathrm{~s})$ & $\log \dot{P}$ & $d(\mathrm{kpc})$ & $\log \varepsilon$ & $\log \eta$ & $\log h$ \\
\hline $\mathrm{J} 1914+1122$ & 0.600997 & -15.1831 & 3.8 & -10.7608 & -16.1911 & -34.272 \\
\hline $\mathrm{J} 1915+0227$ & 0.317306 & -15.5243 & 7.01 & -11.3794 & -16.255 & -34.6017 \\
\hline $\mathrm{J} 1915+0738$ & 1.5427 & -14.4802 & 1.98 & -9.64844 & -15.8976 & -33.6953 \\
\hline $\mathrm{J} 1915+0752$ & 2.05831 & -15.857 & 3.56 & -10.9 & -17.3996 & -35.4522 \\
\hline $\mathrm{J} 1915+0838$ & 0.342777 & -14.8041 & 8.12 & -10.6256 & -15.5683 & -33.9789 \\
\hline $\mathrm{J} 1915+1009$ & 0.404547 & -13.8153 & 7 & -9.56489 & -14.6514 & -32.9976 \\
\hline $\mathrm{J} 1915+1410$ & 0.297494 & -16.3107 & 7.76 & -12.1938 & -17.0133 & -35.4042 \\
\hline $\mathrm{J} 1915+1606$ & 0.05903 & -17.064 & 7.13 & -13.6495 & -17.0642 & -35.4184 \\
\hline $\mathrm{J} 1915+1647$ & 1.61623 & -15.3925 & 3.16 & -10.5406 & -16.8302 & -34.8309 \\
\hline $\mathrm{J} 1916+0748$ & 0.541752 & -13.9706 & 8.24 & -9.59336 & -14.9336 & -33.3506 \\
\hline $\mathrm{J} 1916+0844$ & 0.439995 & -14.5376 & 7.98 & -10.2507 & -15.4102 & -33.8133 \\
\hline $\mathrm{J} 1916+0852$ & 2.18275 & -13.8827 & 6.98 & -8.90027 & -15.4509 & -33.7958 \\
\hline $\mathrm{J} 1916+0951$ & 0.270254 & -14.5986 & 2.88 & -10.5234 & -15.2595 & -33.22 \\
\hline $\mathrm{J} 1916+1023$ & 1.61834 & -15.1669 & 6.93 & -10.3143 & -16.6051 & -34.9469 \\
\hline $\mathrm{J} 1916+1030$ & 0.62897 & -16.4685 & 7.55 & -12.0264 & -17.4963 & -35.8753 \\
\hline $\mathrm{J} 1916+1225$ & 0.227387 & -13.6289 & 6.19 & -9.62871 & -14.2148 & -32.5076 \\
\hline $\mathrm{J} 1916+1312$ & 0.281842 & -14.4377 & 4.5 & -10.3442 & -15.1169 & -33.2711 \\
\hline $\mathrm{J} 1917+0834$ & 2.12967 & -13.757 & 1.51 & -8.7852 & -15.3144 & -32.9945 \\
\hline $\mathrm{J} 1917+1353$ & 0.194631 & -14.1427 & 5 & -10.21 & -14.661 & -32.8611 \\
\hline $\mathrm{J} 1917+1737$ & 0.334725 & -15.4895 & 10.21 & -11.3213 & -16.2433 & -34.7534 \\
\hline $\mathrm{J} 1917+2224$ & 0.425897 & -14.5436 & 8.04 & -10.2709 & -15.4021 & -33.8084 \\
\hline J1918-0642 & 0.007646 & -19.5901 & 0.91 & -17.0632 & -18.7026 & -36.1627 \\
\hline J1918-1052 & 0.798693 & -15.063 & 2.78 & -10.5172 & -16.1945 & -34.1396 \\
\hline $\mathrm{J} 1918+1444$ & 1.18102 & -12.6737 & 1.41 & -7.95795 & -13.9751 & -31.6253 \\
\hline $\mathrm{J} 1918+1541$ & 0.370883 & -14.5952 & 0.68 & -10.3825 & -15.3936 & -32.7271 \\
\hline $\mathrm{J} 1919+0021$ & 1.27226 & -14.1152 & 3.32 & -9.36718 & -15.4489 & -33.4711 \\
\hline $\mathrm{J} 1919+0134$ & 1.60398 & -15.2299 & 10.23 & -10.3812 & -16.6642 & -35.1752 \\
\hline $\mathrm{J} 1919+1314$ & 0.5714 & -14.4214 & 20.84 & -10.021 & -15.4074 & -34.2274 \\
\hline $\mathrm{J} 1919+1645$ & 0.56279 & -15.6655 & 9.56 & -11.2717 & -16.645 & -35.1266 \\
\hline $\mathrm{J} 1919+1745$ & 2.08134 & -14.767 & 6.86 & -9.80521 & -16.3145 & -34.6519 \\
\hline $\mathrm{J} 1920+1040$ & 2.2158 & -14.1884 & 7.19 & -9.19944 & -15.7631 & -34.1209 \\
\hline $\mathrm{J} 1920+1110$ & 0.509886 & -15.8069 & 5.01 & -11.4559 & -16.7435 & -34.9444 \\
\hline $\mathrm{J} 1920+2650$ & 0.785522 & -16.4634 & 1.46 & -11.9248 & -17.5877 & -35.2532 \\
\hline $\mathrm{J} 1921+0812$ & 0.210648 & -14.2708 & 3.47 & -10.3038 & -14.8235 & -32.8649 \\
\hline $\mathrm{J} 1921+1419$ & 0.618183 & -14.2518 & 4.08 & -9.81724 & -15.2721 & -33.3838 \\
\hline $\mathrm{J} 1921+1544$ & 0.143576 & -15.0088 & 15.1 & -11.2082 & -15.395 & -34.075 \\
\hline $\mathrm{J} 1921+1948$ & 0.821035 & -15.0477 & 8.15 & -10.4899 & -16.1912 & -34.6034 \\
\hline $\mathrm{J} 1921+2003$ & 0.760681 & -16.301 & 5.16 & -11.7764 & -17.4114 & -35.6251 \\
\hline $\mathrm{J} 1921+2153$ & 1.3373 & -14.8697 & 0.3 & -10.1 & -16.225 & -33.2032 \\
\hline $\mathrm{J} 1922+1733$ & 0.236171 & -13.8729 & 10 & -9.85622 & -14.4753 & -32.9763 \\
\hline $\mathrm{J} 1922+2018$ & 1.17276 & -15.1878 & 10.74 & -10.4751 & -16.4861 & -35.0182 \\
\hline $\mathrm{J} 1922+2110$ & 1.07792 & -14.0872 & 4 & -9.41121 & -15.349 & -33.4521 \\
\hline $\mathrm{J} 1923+1706$ & 0.547209 & -16.3665 & 6.38 & -11.9849 & -17.3338 & -35.6397 \\
\hline $\mathrm{J} 1923+2515$ & 0.003788 & -20.0259 & 0.99 & -17.8041 & -18.8335 & -36.3302 \\
\hline $\mathrm{J} 1924+1631$ & 2.93519 & -12.4389 & 28.35 & -7.32781 & -14.1357 & -33.0893 \\
\hline $\mathrm{J} 1924+1639$ & 0.158043 & -14.5918 & 9.13 & -10.7495 & -15.0197 & -33.4812 \\
\hline $\mathrm{J} 1924+2040$ & 0.23779 & -14.6799 & 10.75 & -10.6602 & -15.2852 & -33.8177 \\
\hline
\end{tabular}


Table 1 continued

\begin{tabular}{|c|c|c|c|c|c|c|}
\hline Pulsar & $P(\mathrm{~s})$ & $\log \dot{P}$ & $d(\mathrm{kpc})$ & $\log \varepsilon$ & $\log \eta$ & $\log h$ \\
\hline $\mathrm{J} 1926+0431$ & 1.07408 & -14.6091 & 3.95 & -9.93458 & -15.8692 & -33.9669 \\
\hline J1926+0737 & 0.318062 & -15.426 & 5.61 & -11.28 & -16.1576 & -34.4077 \\
\hline J1926-1314 & 4.86428 & -13.4389 & 2.09 & -8.10843 & -15.3551 & -33.1763 \\
\hline $\mathrm{J} 1926+1434$ & 1.32492 & -15.6596 & 8.02 & -10.8939 & -17.0109 & -35.4161 \\
\hline J1926+1648 & 0.579823 & -13.7447 & 6 & -9.33798 & -14.7372 & -33.0164 \\
\hline J1926+1928 & 1.34601 & -14.8447 & 30 & -10.0722 & -16.2029 & -35.181 \\
\hline $\mathrm{J} 1926+2016$ & 0.299072 & -14.4559 & 11.85 & -10.3367 & -15.1609 & -33.7356 \\
\hline $\mathrm{J} 1927+0911$ & 0.290305 & -16.1972 & 7.19 & -12.0909 & -16.8892 & -35.247 \\
\hline $\mathrm{J} 1927+1852$ & 0.482766 & -15.9355 & 10.28 & -11.6084 & -16.8484 & -35.3615 \\
\hline $\mathrm{J} 1927+1856$ & 0.298313 & -14.6498 & 4.81 & -10.5316 & -15.3536 & -33.5368 \\
\hline $\mathrm{J} 1927+2234$ & 1.43107 & -15.1129 & 9.3 & -10.3138 & -16.4978 & -34.9673 \\
\hline $\mathrm{J} 1928+1746$ & 0.06873 & -13.8794 & 8.12 & -10.3988 & -13.9457 & -32.3563 \\
\hline $\mathrm{J} 1928+1923$ & 0.81733 & -14.1972 & 30 & -9.64138 & -15.3388 & -34.317 \\
\hline J1929+1844 & 1.22047 & -14.6271 & 5.34 & -9.89711 & -15.9428 & -34.1714 \\
\hline J1929+1955 & 0.257832 & -14.5918 & 12.08 & -10.537 & -15.2322 & -33.8154 \\
\hline $\mathrm{J} 1929+2121$ & 0.723599 & -14.6696 & 3.36 & -10.1666 & -15.7582 & -33.7856 \\
\hline $\mathrm{J} 1930+1316$ & 0.760032 & -14.4365 & 8.28 & -9.91223 & -15.5465 & -33.9656 \\
\hline J1930-1852 & 0.18552 & -16.7447 & 2.34 & -12.8329 & -17.2423 & -35.1125 \\
\hline $\mathrm{J} 1930+1852$ & 0.136855 & -12.1244 & 7 & -8.34465 & -12.4898 & -30.8359 \\
\hline $\mathrm{J} 1931+1536$ & 0.314355 & -14.3002 & 6.41 & -10.1593 & -15.0267 & -33.3346 \\
\hline $\mathrm{J} 1931+1952$ & 0.501123 & -15.9957 & 22.62 & -11.6523 & -16.9248 & -35.7803 \\
\hline $\mathrm{J} 1932+1059$ & 0.226518 & -14.9355 & 0.31 & -10.937 & -15.5198 & -32.5122 \\
\hline $\mathrm{J} 1932+2020$ & 0.268217 & -14.3747 & 5 & -10.3027 & -15.0323 & -33.2324 \\
\hline $\mathrm{J} 1932+2220$ & 0.14447 & -13.2396 & 10.9 & -9.43635 & -13.6285 & -32.167 \\
\hline J1932-3655 & 0.57142 & -15.5467 & 4.41 & -11.1463 & -16.5328 & -34.6783 \\
\hline $\mathrm{J} 1933+0758$ & 0.437454 & -15.6615 & 8.33 & -11.3772 & -16.5316 & -34.9533 \\
\hline $\mathrm{J} 1933+1304$ & 0.928324 & -15.4976 & 8.1 & -10.8864 & -16.6944 & -35.104 \\
\hline $\mathrm{J} 1933+2421$ & 0.81369 & -14.091 & 5.52 & -9.53707 & -15.2306 & -33.4736 \\
\hline J1933-6211 & 0.003543 & -20.4318 & 0.63 & -18.239 & -19.2103 & -36.5107 \\
\hline $\mathrm{J} 1934+2352$ & 0.178432 & -12.8827 & 23.98 & -8.9878 & -13.3633 & -32.2443 \\
\hline $\mathrm{J} 1935+1616$ & 0.358738 & -14.2218 & 3.7 & -10.0236 & -15.0058 & -33.075 \\
\hline $\mathrm{J} 1935+1745$ & 0.654408 & -15.4225 & 9.57 & -10.9632 & -16.4675 & -34.9495 \\
\hline $\mathrm{J} 1935+2025$ & 0.080118 & -13.2161 & 8.64 & -9.66891 & -13.349 & -31.7865 \\
\hline J1936+1536 & 0.967338 & -14.3936 & 8.41 & -9.76459 & -15.6083 & -34.0342 \\
\hline $\mathrm{J} 1937+1505$ & 2.87277 & -14.251 & 14.07 & -9.14928 & -15.9385 & -34.5878 \\
\hline $\mathrm{J} 1937+2544$ & 0.20098 & -15.1918 & 2.76 & -11.2452 & -15.7241 & -33.6661 \\
\hline $\mathrm{J} 1937+2950$ & 1.65743 & -14.4584 & 6.44 & -9.59553 & -15.907 & -34.2169 \\
\hline $\mathrm{J} 1938+0650$ & 1.12156 & -14.2457 & 3.64 & -9.55238 & -15.5246 & -33.5868 \\
\hline $\mathrm{J} 1938+2010$ & 0.687082 & -14.4685 & 14.36 & -9.98806 & -15.5347 & -34.1929 \\
\hline $\mathrm{J} 1938+2213$ & 0.166116 & -13.3726 & 4.54 & -9.50877 & -13.8222 & -31.9803 \\
\hline $\mathrm{J} 1939+2134$ & 0.001558 & -18.9788 & 1.5 & -17.1428 & -17.4005 & -35.0777 \\
\hline $\mathrm{J} 1939+2449$ & 0.645302 & -13.7375 & 7.34 & -9.28433 & -14.7765 & -33.1432 \\
\hline $\mathrm{J} 1940+2337$ & 0.546824 & -13.1146 & 9.87 & -8.73334 & -14.0816 & -32.577 \\
\hline $\mathrm{J} 1941+0121$ & 0.217317 & -15.719 & 2.89 & -11.7384 & -16.2852 & -34.2472 \\
\hline $\mathrm{J} 1941+1026$ & 0.905394 & -15.0017 & 8.6 & -10.4015 & -16.1877 & -34.6233 \\
\hline $\mathrm{J} 1941+1341$ & 0.559084 & -14.9066 & 8.55 & -10.5156 & -15.8832 & -34.3162 \\
\hline $\mathrm{J} 1941+2525$ & 2.30615 & -12.7932 & 15.35 & -7.78683 & -14.3852 & -33.0724 \\
\hline
\end{tabular}


Table 1 continued

\begin{tabular}{|c|c|c|c|c|c|c|}
\hline Pulsar & $P(\mathrm{~s})$ & $\log \dot{P}$ & $d(\mathrm{kpc})$ & $\log \varepsilon$ & $\log \eta$ & $\log h$ \\
\hline J1941-2602 & 0.402858 & -15.0195 & 4.74 & -10.7709 & -15.8538 & -34.0307 \\
\hline $\mathrm{J} 1942+1743$ & 0.696262 & -15.9957 & 9.83 & -11.5095 & -17.0676 & -35.5612 \\
\hline $\mathrm{J} 1943+0609$ & 0.446226 & -15.3316 & 3.89 & -11.0386 & -16.2103 & -34.3013 \\
\hline J1943-1237 & 0.972429 & -14.7799 & 1.64 & -10.1486 & -15.9969 & -33.7128 \\
\hline $\mathrm{J} 1943+2210$ & 0.005084 & -20.056 & 8.35 & -17.7064 & -18.9914 & -37.4141 \\
\hline J1944+0907 & 0.005185 & -19.767 & 1.28 & -17.4088 & -18.7109 & -36.3192 \\
\hline J1944-1750 & 0.841158 & -15.0061 & 5.3 & -10.4378 & -16.1601 & -34.3855 \\
\hline J1944+1755 & 1.9969 & -15.1367 & 9.5 & -10.1929 & -16.6662 & -35.145 \\
\hline $\mathrm{J} 1944+2236$ & 0.003618 & -20.1273 & 8.46 & -17.9253 & -18.9149 & -37.3433 \\
\hline $\mathrm{J} 1945-0040$ & 1.04563 & -15.2716 & 3.53 & -10.6088 & -16.5202 & -34.569 \\
\hline $\mathrm{J} 1945+1834$ & 1.06871 & -15.6162 & 12.77 & -10.9439 & -16.8742 & -35.4814 \\
\hline J1946-1312 & 0.491865 & -14.7011 & 5.64 & -10.3658 & -15.6221 & -33.8745 \\
\hline $\mathrm{J} 1946+1805$ & 0.440618 & -16.618 & 0.3 & -12.3305 & -17.4912 & -34.4694 \\
\hline $\mathrm{J} 1946+2244$ & 1.33445 & -15.0511 & 7.09 & -10.2823 & -16.4055 & -34.7573 \\
\hline $\mathrm{J} 1946+2535$ & 0.515167 & -14.2487 & 9.58 & -9.89332 & -15.1898 & -33.6722 \\
\hline $\mathrm{J} 1946+2611$ & 0.43506 & -13.6576 & 7.78 & -9.37558 & -14.5253 & -32.9173 \\
\hline J1946-2913 & 0.959448 & -14.8268 & 4.31 & -10.2013 & -16.038 & -34.1735 \\
\hline $\mathrm{J} 1946+3417$ & 0.00317 & -20.4318 & 6.41 & -18.2873 & -19.162 & -37.4699 \\
\hline $\mathrm{J} 1947+0915$ & 1.48074 & -15.3206 & 5.8 & -10.5066 & -16.7202 & -34.9847 \\
\hline J1947+1957 & 0.157509 & -15.2815 & 9.51 & -11.4407 & -15.708 & -34.1872 \\
\hline $\mathrm{J} 1948+2551$ & 0.196627 & -14.0448 & 11.85 & -10.1077 & -14.5676 & -33.1424 \\
\hline $\mathrm{J} 1948+3540$ & 0.717311 & -14.1512 & 7.87 & -9.65204 & -15.2361 & -33.6331 \\
\hline $\mathrm{J} 1949+2306$ & 1.31937 & -15.9101 & 8.65 & -11.1463 & -17.2596 & -35.6977 \\
\hline J1949-2524 & 0.957617 & -14.4855 & 1.34 & -9.86081 & -15.6958 & -33.324 \\
\hline J1949+3106 & 0.013138 & -19.0273 & 7.78 & -16.2654 & -18.375 & -36.7671 \\
\hline $\mathrm{J} 1950+2414$ & 0.004305 & -19.7235 & 7.29 & -17.4461 & -18.5867 & -36.9504 \\
\hline $\mathrm{J} 1951+1123$ & 5.09408 & -14.5186 & 1.66 & -9.16804 & -16.4548 & -34.1759 \\
\hline $\mathrm{J} 1952+1410$ & 0.275026 & -15.8928 & 1.66 & -11.81 & -16.5613 & -34.2825 \\
\hline $\mathrm{J} 1952+2630$ & 0.020732 & -17.3696 & 13.67 & -14.4095 & -16.9154 & -35.5522 \\
\hline $\mathrm{J} 1952+3252$ & 0.039531 & -14.2336 & 3 & -10.9932 & -14.0597 & -32.0379 \\
\hline $\mathrm{J} 1953+1149$ & 0.85189 & -14.5436 & 12.53 & -9.9698 & -15.7032 & -34.3022 \\
\hline $\mathrm{J} 1953+2732$ & 1.33397 & -14.752 & 7.86 & -9.98343 & -16.1063 & -34.5028 \\
\hline J1954+2923 & 0.426677 & -17.767 & 0.42 & -13.4935 & -18.6263 & -35.7506 \\
\hline $\mathrm{J} 1955+2527$ & 0.004873 & -20.04 & 9.06 & -17.7088 & -18.9569 & -37.4151 \\
\hline $\mathrm{J} 1955+2908$ & 0.006133 & -19.5272 & 5.39 & -17.0961 & -18.5441 & -36.7767 \\
\hline $\mathrm{J} 1955+5059$ & 0.518938 & -14.8633 & 1.8 & -10.5047 & -15.8075 & -33.5639 \\
\hline $\mathrm{J} 1956+0838$ & 0.303911 & -15.6576 & 4.31 & -11.5314 & -16.3695 & -34.505 \\
\hline $\mathrm{J} 1957+2831$ & 0.307683 & -14.5072 & 6.98 & -10.3757 & -15.2245 & -33.5694 \\
\hline $\mathrm{J} 1959+2048$ & 0.001607 & -19.7721 & 1.53 & -17.9226 & -18.2073 & -35.893 \\
\hline $\mathrm{J} 1959+3620$ & 0.406081 & -16.4437 & 29.32 & -12.1916 & -17.2815 & -36.2497 \\
\hline $\mathrm{J} 2002+1637$ & 0.276497 & -15.6478 & 6.1 & -11.5627 & -16.3187 & -34.605 \\
\hline $\mathrm{J} 2002+3217$ & 0.696761 & -12.9788 & 6.68 & -8.49227 & -14.051 & -32.3769 \\
\hline $\mathrm{J} 2002+4050$ & 0.905067 & -14.7595 & 8.3 & -10.1593 & -15.9453 & -34.3654 \\
\hline $\mathrm{J} 2004+3137$ & 2.11126 & -13.1273 & 8 & -8.15927 & -14.681 & -33.0851 \\
\hline $\mathrm{J} 2004+3429$ & 0.240953 & -12.684 & 22.18 & -8.65864 & -13.2951 & -32.1421 \\
\hline $\mathrm{J} 2005-0020$ & 2.27966 & -13.5901 & 2.16 & -8.58874 & -15.1771 & -33.0126 \\
\hline $\mathrm{J} 2005+3547$ & 0.615034 & -15.5513 & 30 & -11.1189 & -16.5693 & -35.5475 \\
\hline
\end{tabular}


Table 1 continued

\begin{tabular}{|c|c|c|c|c|c|c|}
\hline Pulsar & $P(\mathrm{~s})$ & $\log \dot{P}$ & $d(\mathrm{kpc})$ & $\log \varepsilon$ & $\log \eta$ & $\log h$ \\
\hline $\mathrm{J} 2005+3552$ & 0.307943 & -14.5243 & 30 & -10.3924 & -15.2419 & -34.2201 \\
\hline J2006-0807 & 0.580871 & -16.3372 & 2.01 & -11.9297 & -17.3305 & -35.1347 \\
\hline $\mathrm{J} 2006+3102$ & 0.163695 & -13.6038 & 5.58 & -9.74631 & -14.047 & -32.2947 \\
\hline $\mathrm{J} 2007+0809$ & 0.325724 & -15.8633 & 3.44 & -11.707 & -16.6053 & -34.6429 \\
\hline $\mathrm{J} 2007+0910$ & 0.458735 & -15.4789 & 2.93 & -11.1738 & -16.3696 & -34.3375 \\
\hline $\mathrm{J} 2007+2722$ & 0.024497 & -18.0173 & 6.85 & -14.9847 & -17.6355 & -35.9723 \\
\hline $\mathrm{J} 2007+3120$ & 0.608205 & -13.8069 & 7.28 & -9.37937 & -14.8201 & -33.1833 \\
\hline $\mathrm{J} 2008+2513$ & 0.589196 & -14.2676 & 3.22 & -9.85389 & -15.267 & -33.2759 \\
\hline $\mathrm{J} 2009+3326$ & 1.43837 & -14.8327 & 10.73 & -10.0314 & -16.2197 & -34.7514 \\
\hline $\mathrm{J} 2010-1323$ & 0.005223 & -20.317 & 1.29 & -17.9556 & -19.264 & -36.8757 \\
\hline $\mathrm{J} 2010+2845$ & 0.565369 & -16.0424 & 6.03 & -11.6466 & -17.0239 & -35.3052 \\
\hline $\mathrm{J} 2010+3230$ & 1.44245 & -14.4413 & 18.89 & -9.63874 & -15.8295 & -34.6068 \\
\hline $\mathrm{J} 2011+3331$ & 0.931733 & -14.7471 & 12.95 & -10.1344 & -15.9456 & -34.5589 \\
\hline J2012-2029 & 0.544002 & -15.2612 & 3.92 & -10.8822 & -16.226 & -34.3203 \\
\hline J2013-0649 & 0.580187 & -15.2211 & 4.85 & -10.8141 & -16.2138 & -34.4006 \\
\hline $\mathrm{J} 2013+3058$ & 0.276028 & -15.8182 & 6.88 & -11.7338 & -16.4883 & -34.8269 \\
\hline $\mathrm{J} 2013+3845$ & 0.230194 & -14.0531 & 13.07 & -10.0475 & -14.6443 & -33.2616 \\
\hline $\mathrm{J} 2015+2524$ & 2.3033 & -15.2924 & 0.69 & -10.2866 & -16.8839 & -34.2238 \\
\hline $\mathrm{J} 2016+1948$ & 0.06494 & -18.3969 & 1.83 & -14.9409 & -18.4385 & -36.202 \\
\hline $\mathrm{J} 2017+0603$ & 0.002896 & -20.0809 & 1.32 & -17.9757 & -18.7719 & -36.3935 \\
\hline $\mathrm{J} 2017+2043$ & 0.537143 & -15.0017 & 3.55 & -10.6282 & -15.961 & -34.0123 \\
\hline $\mathrm{J} 2018+2839$ & 0.557953 & -15.8297 & 0.98 & -11.4397 & -16.8055 & -34.2978 \\
\hline $\mathrm{J} 2018+3431$ & 0.387664 & -14.7352 & 8.79 & -10.5033 & -15.5528 & -33.9978 \\
\hline $\mathrm{J} 2019+2425$ & 0.003935 & -20.1537 & 0.91 & -17.9153 & -18.9778 & -36.4379 \\
\hline $\mathrm{J} 2021+3651$ & 0.103741 & -13.0191 & 1.8 & -9.35968 & -13.2642 & -31.0205 \\
\hline $\mathrm{J} 2021+4026$ & 0.265318 & -13.262 & 2.15 & -9.19479 & -13.9149 & -31.7484 \\
\hline $\mathrm{J} 2022+2854$ & 0.343402 & -14.7235 & 2.1 & -10.5443 & -15.4885 & -33.3118 \\
\hline $\mathrm{J} 2022+3842$ & 0.048579 & -13.065 & 10 & -9.7351 & -12.9806 & -31.4817 \\
\hline $\mathrm{J} 2022+5154$ & 0.529197 & -14.5143 & 1.8 & -10.1472 & -15.467 & -33.2234 \\
\hline $\mathrm{J} 2023+5037$ & 0.372619 & -14.6003 & 1.8 & -10.3856 & -15.4007 & -33.1571 \\
\hline $\mathrm{J} 2027+2146$ & 0.398173 & -15.6925 & 10.09 & -11.449 & -16.5217 & -35.0267 \\
\hline $\mathrm{J} 2027+4557$ & 1.09965 & -15.51 & 22.99 & -10.8253 & -16.7804 & -35.643 \\
\hline $\mathrm{J} 2029+3744$ & 1.2168 & -13.9101 & 7 & -9.18142 & -15.2245 & -33.5706 \\
\hline $\mathrm{J} 2030+2228$ & 0.630513 & -15.0531 & 4.75 & -10.6099 & -16.0819 & -34.2597 \\
\hline $\mathrm{J} 2030+3641$ & 0.200129 & -14.1871 & 11.17 & -10.2423 & -14.7175 & -33.2667 \\
\hline $\mathrm{J} 2032+4127$ & 0.14325 & -13.9031 & 1.7 & -10.1035 & -14.2883 & -32.0198 \\
\hline $\mathrm{J} 2033+0042$ & 5.0134 & -13.9957 & 2.87 & -8.65209 & -15.925 & -33.8839 \\
\hline $\mathrm{J} 2033+1734$ & 0.005949 & -19.9547 & 1.37 & -17.5368 & -18.9583 & -36.596 \\
\hline $\mathrm{J} 2033-1938$ & 1.28172 & -15.342 & 1.54 & -10.5907 & -16.6789 & -34.3675 \\
\hline $\mathrm{J} 2036+2835$ & 1.35873 & -14.6799 & 6.78 & -9.90327 & -16.0421 & -34.3744 \\
\hline $\mathrm{J} 2037+1942$ & 2.07438 & -14.6904 & 2.05 & -9.73003 & -16.2364 & -34.0492 \\
\hline $\mathrm{J} 2037+3621$ & 0.618715 & -14.3468 & 5.05 & -9.91184 & -15.3674 & -33.5718 \\
\hline $\mathrm{J} 2038-3816$ & 1.57729 & -14.384 & 2.94 & -9.54269 & -15.8111 & -33.7805 \\
\hline $\mathrm{J} 2038+5319$ & 1.42457 & -15.025 & 13.83 & -10.2279 & -16.4079 & -35.0497 \\
\hline $\mathrm{J} 2040+1657$ & 0.865606 & -15.2255 & 3.51 & -10.6447 & -16.392 & -34.4383 \\
\hline
\end{tabular}


Table 1 continued

\begin{tabular}{|c|c|c|c|c|c|c|}
\hline Pulsar & $P(\mathrm{~s})$ & $\log \dot{P}$ & $d(\mathrm{kpc})$ & $\log \varepsilon$ & $\log \eta$ & $\log h$ \\
\hline $\mathrm{J} 2043+1711$ & 0.00238 & -20.2807 & 1.25 & -18.2606 & -18.8864 & -36.4844 \\
\hline $\mathrm{J} 2043+2740$ & 0.096131 & -14.8962 & 1.13 & -11.2699 & -15.1082 & -32.6623 \\
\hline $\mathrm{J} 2044+4614$ & 1.39271 & -15.2007 & 26.78 & -10.4133 & -16.5737 & -35.5025 \\
\hline $\mathrm{J} 2045+0912$ & 0.395555 & -15.71 & 1.99 & -11.4693 & -16.5363 & -34.3362 \\
\hline J2046-0421 & 1.54694 & -14.8327 & 3.83 & -9.99976 & -16.2513 & -34.3356 \\
\hline $\mathrm{J} 2046+1540$ & 1.13829 & -15.7399 & 2.56 & -11.0402 & -17.0253 & -34.9346 \\
\hline $\mathrm{J} 2046+5708$ & 0.476735 & -13.9547 & 9.67 & -9.63295 & -14.8621 & -33.3486 \\
\hline $\mathrm{J} 2047+5029$ & 0.445945 & -14.3788 & 5.03 & -10.0861 & -15.2573 & -33.4599 \\
\hline J2048-1616 & 1.96157 & -13.9586 & 0.95 & -9.02255 & -15.4804 & -32.9591 \\
\hline $\mathrm{J} 2048+2255$ & 0.283901 & -16.8182 & 6.18 & -12.7215 & -17.5005 & -35.7925 \\
\hline $\mathrm{J} 2051-0827$ & 0.004509 & -19.8962 & 1.28 & -17.5987 & -18.7794 & -36.3877 \\
\hline $\mathrm{J} 2053-7200$ & 0.341336 & -15.7077 & 1.05 & -11.5311 & -16.4701 & -33.9923 \\
\hline $\mathrm{J} 2055+2209$ & 0.815181 & -14.8729 & 2.15 & -10.3182 & -16.0133 & -33.8468 \\
\hline $\mathrm{J} 2055+3630$ & 0.221508 & -15.433 & 5 & -11.4441 & -16.0075 & -34.2075 \\
\hline $\mathrm{J} 2105+6223$ & 2.30488 & -14.2823 & 3.03 & -9.27623 & -15.8741 & -33.8566 \\
\hline J2108-3429 & 1.4231 & -14.4559 & 2.63 & -9.65924 & -15.8383 & -33.7593 \\
\hline $\mathrm{J} 2108+4441$ & 0.414871 & -16.0645 & 5.28 & -11.8031 & -16.9116 & -35.1352 \\
\hline $\mathrm{J} 2111+2106$ & 3.95385 & -14.4895 & 5.64 & -9.24898 & -16.3156 & -34.568 \\
\hline $\mathrm{J} 2113+2754$ & 1.20285 & -14.5817 & 2.61 & -9.85803 & -15.8911 & -33.8088 \\
\hline $\mathrm{J} 2113+4644$ & 1.01469 & -15.1457 & 4 & -10.4959 & -16.3812 & -34.4843 \\
\hline $\mathrm{J} 2116+1414$ & 0.440153 & -15.5391 & 4.43 & -11.252 & -16.4119 & -34.5593 \\
\hline $\mathrm{J} 2124+1407$ & 0.694053 & -15.1146 & 2.09 & -10.6298 & -16.1852 & -34.0064 \\
\hline $\mathrm{J} 2124-3358$ & 0.004931 & -19.6861 & 0.41 & -17.3497 & -18.6082 & -35.7221 \\
\hline J2127-6648 & 0.325771 & -15.6459 & 2.75 & -11.4895 & -16.388 & -34.3283 \\
\hline J2129-5721 & 0.003726 & -19.6799 & 3.2 & -17.4652 & -18.4802 & -36.4865 \\
\hline J2136-1606 & 1.22724 & -16.7959 & 1.27 & -12.0635 & -18.114 & -35.7188 \\
\hline $\mathrm{J} 2139+2242$ & 1.08351 & -14.8477 & 4.71 & -10.1694 & -16.1117 & -34.2858 \\
\hline J2144-3933 & 8.50983 & -15.3045 & 0.16 & -9.73114 & -17.4636 & -34.1688 \\
\hline $\mathrm{J} 2145-0750$ & 0.016052 & -19.5258 & 0.53 & -16.6768 & -18.9605 & -36.1858 \\
\hline $\mathrm{J} 2149+6329$ & 0.38014 & -15.7747 & 13.65 & -11.5513 & -16.5838 & -35.22 \\
\hline $\mathrm{J} 2150+5247$ & 0.332206 & -13.9957 & 5.67 & -9.83082 & -14.7462 & -33.0009 \\
\hline $\mathrm{J} 2151+2315$ & 0.593534 & -15.15 & 1.42 & -10.7331 & -16.1526 & -33.8059 \\
\hline $\mathrm{J} 2155+2813$ & 1.60902 & -15.0381 & 5.06 & -10.1881 & -16.4738 & -34.679 \\
\hline $\mathrm{J} 2155-3118$ & 1.03 & -14.9066 & 0.97 & -10.2503 & -16.1486 & -33.6364 \\
\hline $\mathrm{J} 2155-5641$ & 1.37365 & -14.3737 & 0.86 & -9.59233 & -15.7407 & -33.1762 \\
\hline $\mathrm{J} 2156+2618$ & 0.498149 & -16.8477 & 4.71 & -12.5069 & -17.7742 & -35.9483 \\
\hline $\mathrm{J} 2157+4017$ & 1.52527 & -14.4647 & 2.9 & -9.63791 & -15.8772 & -33.8407 \\
\hline $\mathrm{J} 2205+1444$ & 0.938014 & -15.0458 & 3.33 & -10.4301 & -16.2471 & -34.2706 \\
\hline $\mathrm{J} 2206+6151$ & 0.322674 & -15.4012 & 13.81 & -11.249 & -16.1391 & -34.7804 \\
\hline $\mathrm{J} 2208+5500$ & 0.933161 & -14.1555 & 3.55 & -9.54211 & -15.3546 & -33.4059 \\
\hline $\mathrm{J} 2212+2933$ & 1.00459 & -15.3054 & 4.76 & -10.66 & -16.5365 & -34.7152 \\
\hline $\mathrm{J} 2214+3000$ & 0.003119 & -19.8539 & 1 & -17.7164 & -18.577 & -36.0781 \\
\hline $\mathrm{J} 2215+1538$ & 0.374196 & -14.6253 & 3.12 & -10.4087 & -15.4275 & -33.4227 \\
\hline $\mathrm{J} 2215+5135$ & 0.00261 & -19.4763 & 3.26 & -17.4162 & -18.122 & -36.1363 \\
\hline $\mathrm{J} 2216+5759$ & 0.419102 & -13.1612 & 7.82 & -8.89538 & -14.0126 & -32.4069 \\
\hline $\mathrm{J} 2217+5733$ & 1.05684 & -15.1864 & 6.82 & -10.519 & -16.4396 & -34.7744 \\
\hline
\end{tabular}


Table 1 continued

\begin{tabular}{|c|c|c|c|c|c|c|}
\hline Pulsar & $P(\mathrm{~s})$ & $\log \dot{P}$ & $d(\mathrm{kpc})$ & $\log \varepsilon$ & $\log \eta$ & $\log h$ \\
\hline $\mathrm{J} 2219+4754$ & 0.538469 & -14.5575 & 2.61 & -10.1829 & -15.5178 & -33.4355 \\
\hline $\mathrm{J} 2222-0137$ & 0.032818 & -19.2321 & 0.27 & -16.0725 & -18.9774 & -35.9098 \\
\hline $\mathrm{J} 2222+2923$ & 0.281399 & -17.2097 & 4.46 & -13.1169 & -17.8882 & -36.0386 \\
\hline $\mathrm{J} 2225+6535$ & 0.682542 & -14.015 & 2 & -9.53744 & -15.0783 & -32.8804 \\
\hline $\mathrm{J} 2229+2643$ & 0.002978 & -20.8356 & 1.43 & -18.7183 & -19.5387 & -37.1951 \\
\hline $\mathrm{J} 2229+6114$ & 0.051624 & -13.1062 & 3 & -9.74993 & -13.0482 & -31.0264 \\
\hline $\mathrm{J} 2229+6205$ & 0.443055 & -14.6459 & 5.7 & -10.356 & -15.5215 & -33.7784 \\
\hline $\mathrm{J} 2234+2114$ & 1.35875 & -15.6536 & 3.38 & -10.8771 & -17.0159 & -35.0459 \\
\hline $\mathrm{J} 2235+1506$ & 0.059767 & -18.8013 & 1.15 & -15.3814 & -18.807 & -36.3687 \\
\hline J2236-5527 & 0.006908 & -20.0177 & 2.03 & -17.5349 & -19.0862 & -36.8948 \\
\hline $\mathrm{J} 2240+5832$ & 0.139935 & -13.8125 & 14.85 & -10.0231 & -14.1876 & -32.8603 \\
\hline $\mathrm{J} 2241-5236$ & 0.002187 & -20.1778 & 0.68 & -18.1945 & -18.7468 & -36.0804 \\
\hline $\mathrm{J} 2242+6950$ & 1.6645 & -14.317 & 2.3 & -9.45222 & -15.7674 & -33.6302 \\
\hline $\mathrm{J} 2243+1518$ & 0.596799 & -15.9469 & 2.9 & -11.5276 & -16.9519 & -34.9154 \\
\hline J2248-0101 & 0.477233 & -15.1811 & 2.28 & -10.8589 & -16.089 & -33.948 \\
\hline $\mathrm{J} 2253+1516$ & 0.792236 & -16.1772 & 2.81 & -11.6349 & -17.3052 & -35.2549 \\
\hline $\mathrm{J} 2257+5909$ & 0.368246 & -14.2403 & 3 & -10.0307 & -15.0356 & -33.0138 \\
\hline $\mathrm{J} 2301+5852$ & 6.97907 & -12.327 & 3.3 & -6.83973 & -14.3999 & -32.4195 \\
\hline $\mathrm{J} 2302+4442$ & 0.005192 & -19.8761 & 0.75 & -17.5174 & -18.8206 & -36.1968 \\
\hline $\mathrm{J} 2302+6028$ & 1.2064 & -14.6904 & 6.8 & -9.96543 & -16.001 & -34.3346 \\
\hline $\mathrm{J} 2305+3100$ & 1.57589 & -14.5391 & 2.61 & -9.69812 & -15.9658 & -33.8835 \\
\hline $\mathrm{J} 2305+4707$ & 1.06637 & -15.2449 & 4.35 & -10.5735 & -16.5019 & -34.6415 \\
\hline $\mathrm{J} 2307+2225$ & 0.535829 & -17.0605 & 0.38 & -12.688 & -18.0187 & -35.0995 \\
\hline $\mathrm{J} 2308+5547$ & 0.475068 & -15.7011 & 2.42 & -11.3809 & -16.6071 & -34.4919 \\
\hline $\mathrm{J} 2313+4253$ & 0.349434 & -15.9508 & 1.06 & -11.764 & -16.7233 & -34.2497 \\
\hline $\mathrm{J} 2317+1439$ & 0.003445 & -20.6162 & 1.89 & -18.4355 & -19.3825 & -37.16 \\
\hline $\mathrm{J} 2317+2149$ & 1.44465 & -14.9788 & 1.45 & -10.1756 & -16.3677 & -34.0301 \\
\hline $\mathrm{J} 2319+6411$ & 0.216018 & -15.7878 & 25.66 & -11.8099 & -16.3515 & -35.2618 \\
\hline $\mathrm{J} 2321+6024$ & 2.25649 & -14.1524 & 2.7 & -9.15554 & -15.735 & -33.6674 \\
\hline $\mathrm{J} 2322+2057$ & 0.004808 & -20.0132 & 0.78 & -17.6878 & -18.9243 & -36.3175 \\
\hline J2324-6054 & 2.34749 & -14.5867 & 0.9 & -9.57264 & -16.1865 & -33.6418 \\
\hline $\mathrm{J} 2325-0530$ & 0.868735 & -14.9872 & 1.07 & -10.4048 & -16.1552 & -33.6856 \\
\hline $\mathrm{J} 2325+6316$ & 1.43631 & -14.5482 & 10.86 & -9.74751 & -15.9346 & -34.4715 \\
\hline $\mathrm{J} 2326+6113$ & 0.233652 & -15.4522 & 4.82 & -11.4402 & -16.0499 & -34.234 \\
\hline J2330-2005 & 1.64362 & -14.3344 & 0.47 & -9.47516 & -15.7794 & -32.9525 \\
\hline $\mathrm{J} 2333+6145$ & 0.756899 & -14.9281 & 5.01 & -10.4056 & -16.0363 & -34.2372 \\
\hline $\mathrm{J} 2337+6151$ & 0.49537 & -12.7144 & 0.7 & -8.37606 & -13.6385 & -30.9847 \\
\hline $\mathrm{J} 2339-0533$ & 0.002884 & -19.8508 & 1.1 & -17.7473 & -18.5399 & -36.0824 \\
\hline J2346-0609 & 1.18146 & -14.8665 & 1.96 & -10.1506 & -16.168 & -33.9613 \\
\hline $\mathrm{J} 2354+6155$ & 0.944784 & -13.7878 & 3.31 & -9.16903 & -14.9923 & -33.0132 \\
\hline
\end{tabular}




\section{References}

1. J.C.N. de Araujo, J.G. Coelho, C.A. Costa, JCAP 7, 023 (2016a)

2. J.C.N. de Araujo, J.G. Coelho, C.A. Costa, EPJC 76, 481 (2016b)

3. P.G. Krastev, B.-A. Li, A. Worley, Phys. Lett. B 668, 1 (2008)

4. S. Bonazzola, J. Frieben, E. Gourgoulhon, ApJ 460, 379 (1996)

5. M. Zimmermann, E. Szedenits Jr., PRD 20, 351 (1979)

6. J.C.N. de Araujo, J.G. Coelho, C.A. Costa, ApJ 831, 35 (2016c)

7. S.L. Shapiro, S. A. Teukolsky, Research supported by the National Science Foundation (Wiley-Interscience, New York, 1983), p. 663
8. S. Bonazzola, E. Gourgoulhon, A\&A 312, 675 (1996)

9. K. Konno, T. Obata, Y. Kojima, A\&A 356, 234 (2000)

10. T. Regimbau, J.A. de Freitas Pacheco, A\&A 447, 1 (2006)

11. J. Aasi, J. Abadie, B.P. Abbott et al., ApJ 785, 119 (2014)

12. B.P. Abbott, R. Abbott, T.D. Abbott, M.R. Abernathy et al., ApJ 839, 1 (2017)

13. S.K. Lander, D.I. Jones, MNRAS 395, 2162 (2009) 